\title{
Carbon Flux to the Atmosphere from Land-Use Changes: 1850 to 1990
}

\author{
Richard A. Houghton \\ J oseph L. Hackler
}

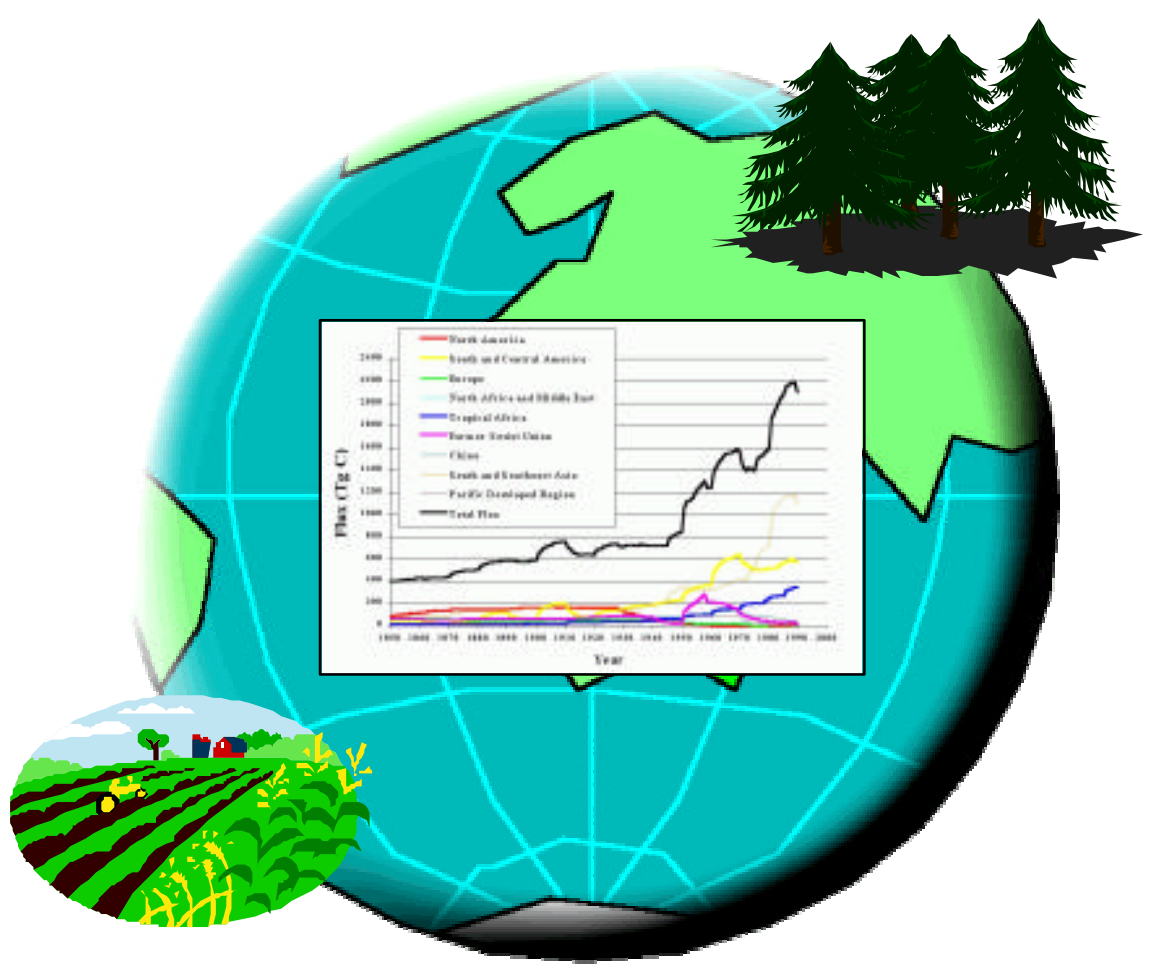

CDIAC

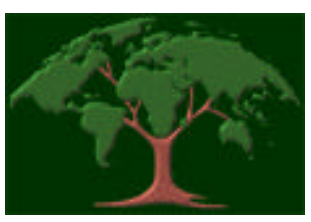


ORNL/CDIAC-131

NDP-050/R1

\title{
CARBON FLUX TO THE ATMOSPHERE FROM LAND-USE CHANGES: 1850 TO 1990
}

\author{
Contributed by \\ Richard A. Houghton \\ Joseph L. Hackler \\ The Woods Hole Research Center \\ Woods Hole, Massachusetts \\ Prepared by \\ Robert M. Cushman \\ Carbon Dioxide Information Analysis Center \\ Environmental Sciences Division \\ Publication No. 5054 \\ Date Published: February 2001
}

Prepared for the

Environmental Sciences Division

Office of Biological and Environmental Research

U.S. Department of Energy

Budget Activity Number KP 1204010

Prepared by the

Carbon Dioxide Information Analysis Center

Environmental Sciences Division

OAK RIDGE NATIONAL LABORATORY

Oak Ridge, Tennessee 37831-6335

managed by

University of Tennessee-Battelle, LLC

for the

U.S. DEPARTMENT OF ENERGY

under contract DE-AC05-00OR22725 
CONTENTS

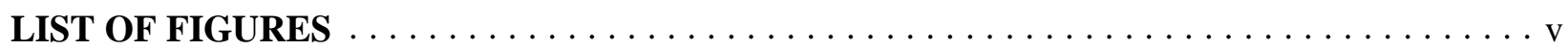

LIST OF TABLES $\ldots \ldots \ldots \ldots \ldots \ldots \ldots \ldots \ldots \ldots \ldots \ldots \ldots \ldots \ldots \ldots \ldots \ldots \ldots$

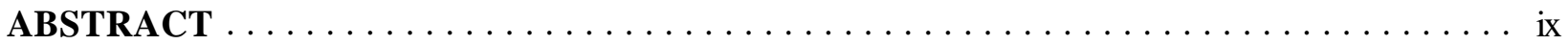

1. BACKGROUND INFORMATION $\ldots \ldots \ldots \ldots \ldots \ldots \ldots \ldots \ldots \ldots \ldots \ldots$

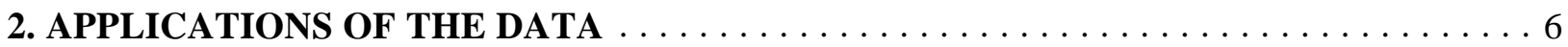

3. DATA LIMITATIONS AND RESTRICTIONS $\ldots \ldots \ldots \ldots \ldots \ldots \ldots \ldots \ldots \ldots$

4. DATA CHECKS AND PROCESSING PERFORMED BY CDIAC $\ldots \ldots \ldots \ldots \ldots \ldots \ldots 7$

5. INSTRUCTIONS FOR OBTAINING THE DATA AND DOCUMENTATION $\ldots \ldots \ldots$.

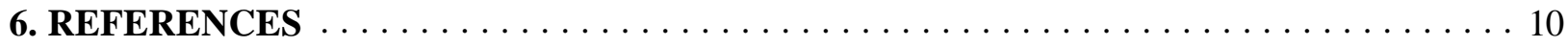

7. LISTING OF FILES PROVIDED $\ldots \ldots \ldots \ldots \ldots \ldots \ldots \ldots \ldots \ldots \ldots \ldots \ldots$

8. DESCRIPTION OF THE DOCUMENTATION FILE $\ldots \ldots \ldots \ldots \ldots \ldots \ldots \ldots \ldots \ldots$

9. DESCRIPTION, FORMAT, AND PARTIAL LISTINGS OF THE ASCII DATA FILES . 12

10. DESCRIPTION AND FORMAT OF THE LOTUS 1-2-3 ${ }^{\circledR}$ BINARY

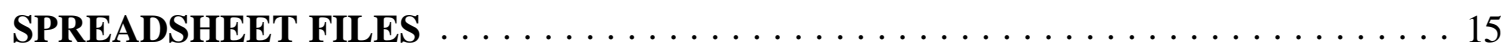

11. SAS $^{\circledR}$ AND FORTRAN CODES TO ACCESS THE DATA $\ldots \ldots \ldots \ldots \ldots \ldots \ldots \ldots$

APPENDIX A. ECOSYSTEM AREA BY REGION $\ldots \ldots \ldots \ldots \ldots \ldots \ldots \ldots \ldots \ldots$ A-1

APPENDIX B. REGIONAL LAND-USE CHANGE AND WOOD HARVEST DATA . . . . . B-1

APPENDIX C. REGIONAL CARBON-CHANGE COEFFICIENTS $\ldots \ldots \ldots \ldots \ldots \ldots$ C-1

APPENDIX D. FULL LISTING OF NDP050.DAT (FILE 2) $\ldots \ldots \ldots \ldots \ldots \ldots \ldots \ldots \ldots$ D-1

APPENDIX E. FULL LISTING OF COMPARE.DAT (FILE 4) $\ldots \ldots \ldots \ldots \ldots \ldots \ldots \ldots$ E-1

APPENDIX F: REPRINT OF PERTINENT LITERATURE $\ldots \ldots \ldots \ldots \ldots \ldots \ldots \ldots$ F-1 
The annual net flux of carbon to the atmosphere from changes in land use 1850-1990,

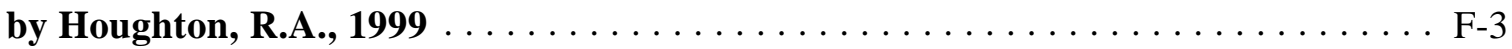




\section{LIST OF FIGURES}

Figure $\quad$ Page

1 Map of the nine regions covered in this database. $\ldots \ldots \ldots \ldots \ldots \ldots \ldots \ldots \ldots$

2 Net flux of carbon to the atmosphere from land-use changes, by region,

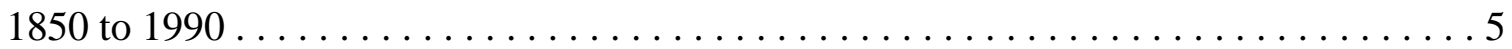

3 Comparison of estimates of net flux of carbon to the atmosphere from

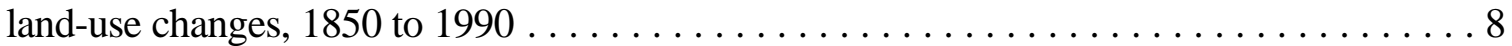




\section{LIST OF TABLES}

Table $\quad$ Page

1 Countries constituting the nine regions covered in this database $\ldots \ldots \ldots \ldots \ldots \ldots \ldots$

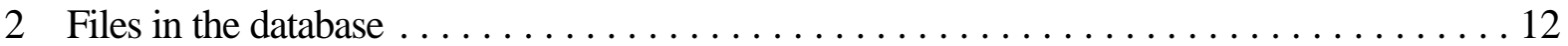

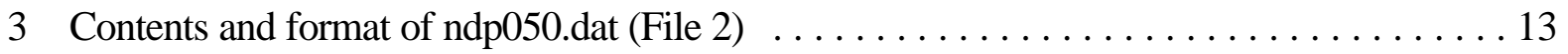

4 Contents and format of compare.dat $($ File 4$) \ldots \ldots \ldots \ldots \ldots \ldots \ldots \ldots \ldots \ldots \ldots$ 


\begin{abstract}
Houghton, R. A., and J. L. Hackler. 2001. Carbon Flux to the Atmosphere from Land-Use Changes: 1850 to 1990. ORNL/CDIAC-131, NDP-050/R1

(http://cdiac.esd.ornl.gov/ndps/ndp050.html). Carbon Dioxide Information Analysis Center, U.S. Department of Energy, Oak Ridge National Laboratory, Oak Ridge, Tennessee, U.S.A. $86 \mathrm{pp}$.
\end{abstract}

The database documented in this numeric data package, a revision to a database originally published by the Carbon Dioxide Information Analysis Center (CDIAC) in 1995, consists of annual estimates, from 1850 through 1990, of the net flux of carbon between terrestrial ecosystems and the atmosphere resulting from deliberate changes in land cover and land use, especially forest clearing for agriculture and the harvest of wood for wood products or energy. The data are provided on a year-by-year basis for nine regions (North America, South and Central America, Europe, North Africa and the Middle East, Tropical Africa, the Former Soviet Union, China, South and Southeast Asia, and the Pacific Developed Region) and the globe. Some data begin earlier than 1850 (e.g., for six regions, areas of different ecosystems are provided for the year 1700) or extend beyond 1990 (e.g., fuelwood harvest in South and Southeast Asia, by forest type, is provided through 1995).

The global net flux during the period 1850 to 1990 was $124 \mathrm{Pg}$ of carbon ( 1 petagram $=10^{15}$ grams). During this period, the greatest regional flux was from South and Southeast Asia (39 Pg of carbon), while the smallest regional flux was from North Africa and the Middle East (3 Pg of carbon). For the year 1990, the global total net flux was estimated to be 2.1 Pg of carbon.

This numeric data package contains a year-by-year regional data set of net flux estimates, a year-byyear data set comparing several estimates of global total net flux, and this documentation file (which includes SAS ${ }^{\circledR 1}$ and Fortran codes to read the ASCII data files). The data files are provided in both flat ASCII and binary spreadsheet format.

The data files and this documentation are available without charge on a variety of media and via the Internet from CDIAC.

Keywords: agriculture, carbon, deforestation, forests, land cover, land use, pastures, plantations, shifting agriculture, soil, vegetation

${ }^{1} \mathrm{SAS}^{\circledR}$ is a registered trademark of the SAS Institute, Inc., Cary, North Carolina 27511. 


\section{BACKGROUND INFORMATION}

In the attempt to "balance" the global carbon cycle (that is, reconcile the known sources and sinks of carbon), two major unknowns remain: the flux between the atmosphere and the oceans and the flux between the atmosphere and terrestrial ecosystems. To address the latter, several investigators have attempted to estimate the flows of carbon between the atmosphere and both temperate and tropical ecosystems.

Quantification of the role of changing land use in the global cycling of carbon (and, consequently, in controlling atmospheric concentrations of carbon dioxide, the single most important greenhouse gas) requires complete, consistent, and accurate databases of vegetation, land use, and biospheric carbon content. The Carbon Dioxide Information Analysis Center (CDIAC) has previously made available several important quality-assured and documented databases on this topic (Olson et al. 1985, Richards and Flint 1994, Houghton and Hackler 1995, and Brown and Gaston 1996).

This database is a revision to Houghton and Hackler (1995). This revised numeric data package provides and documents the data corresponding to the analysis reported by Houghton (1999). It consists of annual estimates, from 1850 through 1990, of the net flux of carbon between terrestrial ecosystems and the atmosphere resulting from deliberate changes in land cover and land use, especially forest clearing for agriculture and the harvest of wood for wood products or energy. The data are provided on a year-by-year basis for nine regions shown in Figure 1 and specified by country in Table 1 (North America, South and Central America, Europe, North Africa and the Middle East, Tropical Africa, the Former Soviet Union, China, South and Southeast Asia, and the Pacific Developed Region) and the globe. Note that South and Central America, Tropical Africa, and South and Southeast Asia, as used in this database, are called Latin America, Sub-Saharan Africa, and Tropical Asia, respectively, in Houghton (1999). Some data begin earlier than 1850 (e.g., for six regions, areas of different ecosystems are provided for the year 1700) or extend beyond 1990 (e.g., fuelwood harvest in South and Southeast Asia, by forest type, is provided through 1995).

The approach used to derive this time series of flux estimates is described fully in Houghton (1999) and other publications (Houghton et al. 1983, 1987; Houghton and Hackler 1995, 1999). The methodology takes into account not only the initial removal and oxidation of the carbon in the vegetation but also subsequent regrowth and changes in soil carbon. The net flux of carbon to the atmosphere from changes in land use from 1850 to 1990 was modeled as a function of documented land-use change and changes in aboveground and belowground carbon following changes in land use. The changes in carbon, with time, following land-use change are specified by region and ecosystem type. 


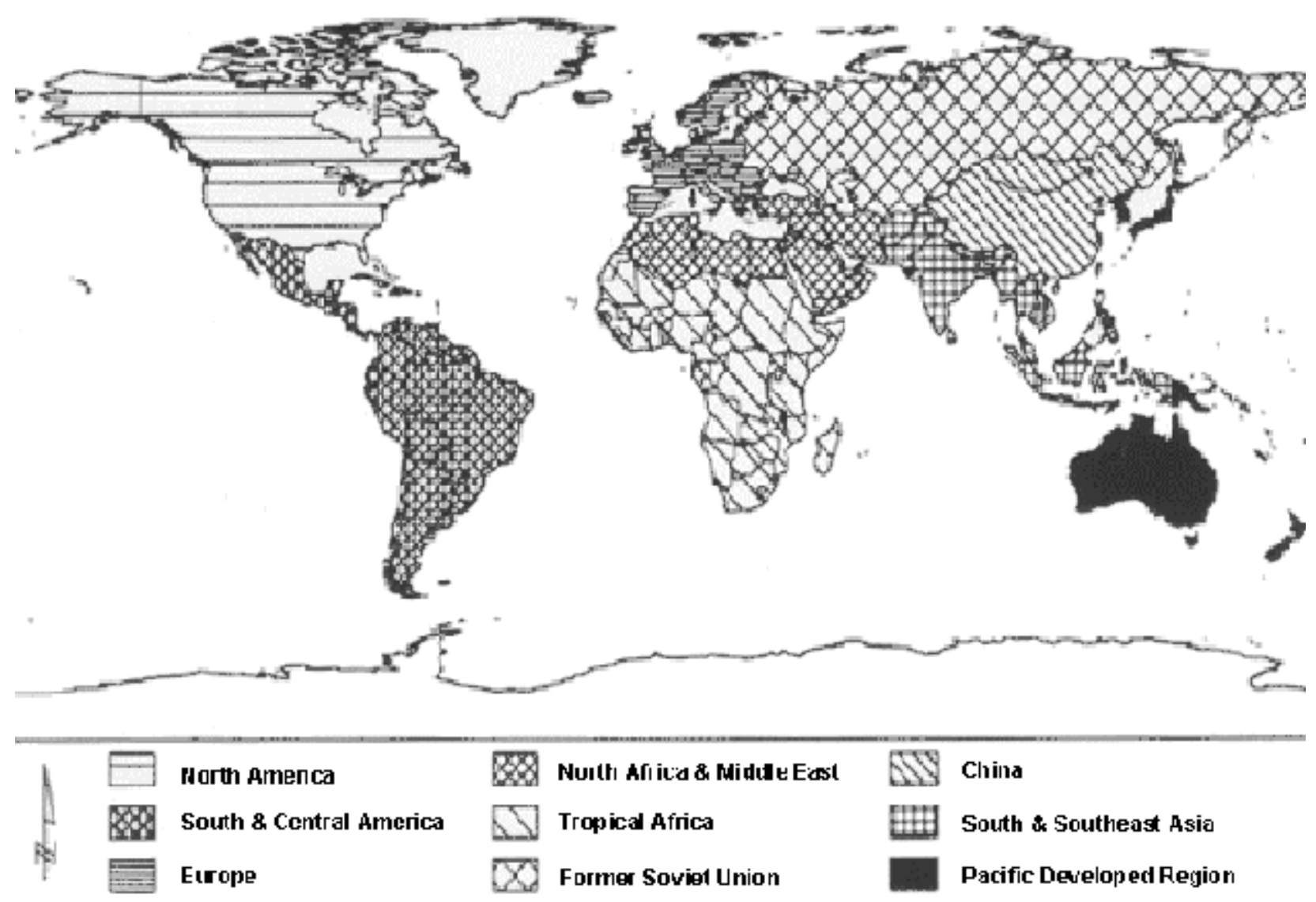

Figure 1. Map of the nine regions covered in this database.

Data on ecosystem areas in each region are listed in Appendix A, which provides areas for the years 1700 (for six of the nine regions), 1850, and 1990, along with the percent change from 1850 to 1990.

Data on changes in land-use and wood harvest are listed in Appendix B, which provides the regional details of fuelwood (nonindustrial logging) and timber (industrial logging) harvest by forest type, changes in area of pasture, forest plantation, afforestation, forest clearing for croplands, and lands in shifting cultivation.

The approach uses a bookkeeping model to track, with an annual time step, changes in aboveground and belowground carbon in different kinds of ecosystems following changes in land use. Annual rates of expansion and contraction of agricultural area (for cropland, pasture, and shifting cultivation) and of wood harvest were used to estimate the types of ecosystem affected and the change in area of each affected ecosystem type. Then, response curves were generated to estimate the changes in carbon, for years to decades, that follow each type of land management or land-use change. All carbon in the affected area is accounted for: live vegetation, soil, slash 
(woody debris produced during disturbance), and wood products. However, this procedure does not account for all processes that affect ecosystem carbon storage and fluxes (e.g., natural disturbances, fire suppression, and environmental factors, such as $\mathrm{CO}_{2}$ and climate, that affect vegetation). Furthermore, the analysis ignores fluxes of carbon to or from ecosystems not directly affected by land-use change. Data on land-use change, wood harvest, and carbon in ecosystems were obtained from a number of sources, detailed in Houghton (1999).

Table 1. Countries constituting the nine regions covered in this database

\begin{tabular}{|c|c|}
\hline Region & Countries \\
\hline North America & Canada, United States \\
\hline $\begin{array}{l}\text { South and Central } \\
\text { America }\end{array}$ & $\begin{array}{l}\text { Argentina, Bahamas, Belize, Bolivia, Brazil, Chile, Colombia, Costa Rica, Cuba, } \\
\text { Dominican Republic, Ecuador, El Salvador, Falkland Islands, French Guiana, } \\
\text { Guadeloupe, Guatemala, Guyana, Haiti, Honduras, Jamaica, Martinique, Mexico, } \\
\text { Nicaragua, Panama, Paraguay, Peru, Puerto Rico, Surinam, Trinidad \& Tobago, } \\
\text { Uruguay, Venezuela }\end{array}$ \\
\hline Europe & $\begin{array}{l}\text { Albania, Andorra, Austria, Belgium, Bulgaria, Czechoslovakia, Denmark, Finland, } \\
\text { France, Germany, Great Britain, Greece, Hungary, Iceland, Ireland, Italy, Liechtenstein, } \\
\text { Luxembourg, Malta, Monaco, Netherlands, Norway, Poland, Portugal, Romania, San } \\
\text { Marino, Spain, Switzerland, Yugoslavia }\end{array}$ \\
\hline $\begin{array}{l}\text { North Africa and the } \\
\text { Middle East }\end{array}$ & $\begin{array}{l}\text { Afghanistan, Algeria, Bahrain, Cyprus, Democratic Yemen, Egypt, Iran, Iraq, Israel, } \\
\text { Jordan, Kuwait, Lebanon, Libya, Morocco, Oman, Qatar, Saudi Arabia, Syria, Tunisia, } \\
\text { Turkey, United Arab Emirates, Yemen }\end{array}$ \\
\hline Tropical Africa & $\begin{array}{l}\text { Angola, Benin, Botswana, Burundi, Cabinda, Cameroon, Central African Republic, } \\
\text { Chad, Djibouti, Equatorial Guinea, Ethiopia, Gabon, Gambia, Ghana, Guinea, Guinea- } \\
\text { Bissau, Ivory Coast, Kenya, Lesotho, Liberia, Madagascar, Malawi, Mali, Mauritania, } \\
\text { Mauritius, Mozambique, Namibia, Niger, Nigeria, Republic of Congo, Reunion, Rio } \\
\text { Muni, Rwanda, Senegal, Sierra Leone, Somalia, South Africa, Sudan, Swaziland, } \\
\text { Tanzania, Togo, Uganda, Upper Volta, Western Sahara, Zaire, Zambia, Zimbabwe }\end{array}$ \\
\hline Former Soviet Union & $\begin{array}{l}\text { Armenia, Azerbaijan, Byelorussia, Estonia, Georgia, Kazakhstan, Kirghistan, Latvia, } \\
\text { Lithuania, Moldavia, Russia, Tajikistan, Turkmenistan, Ukraine, Uzbekistan }\end{array}$ \\
\hline China & People's Republic of China, Mongolia \\
\hline $\begin{array}{l}\text { South and Southeast } \\
\text { Asia }\end{array}$ & $\begin{array}{l}\text { Bangladesh, Bhutan, Brunei, Burma, Cambodia, India, Indonesia, Laos, Malaysia, Nepal, } \\
\text { Pakistan, Philippines, Sri Lanka, Thailand, Vietnam }\end{array}$ \\
\hline $\begin{array}{l}\text { Pacific Developed } \\
\text { Region }\end{array}$ & $\begin{array}{l}\text { Australia, Japan, New Zealand, North Korea, Oceania, Papua New Guinea, South Korea, } \\
\text { Taiwan }\end{array}$ \\
\hline
\end{tabular}


The bookkeeping model partitioned the vegetation after land-use change into three pools: standing live vegetation, dead material left on-site, and woody material removed from the site. The model tracked the return of carbon in the remaining live vegetation to pre-disturbance values. Dead material left on-site and woody material removed from the site (e.g., for timber or firewood) decayed at specified rates. Changes in soil carbon included both post-disturbance losses and eventual recoveries. The coefficients and time constants were specified by region, ecosystem type, and land-use type (see App. C, which provides details of changes in carbon in vegetation and soils with time as a result of land-use change). Finally, changes in on-site carbon pools and carbon in off-site wood products were used to estimate fluxes to and from the atmosphere.

The estimated global total net flux of carbon from changes in land use increased from $397 \mathrm{Tg}$ of carbon ( 1 teragram $=10^{12}$ gram $)$ in 1850 to $2187 \mathrm{Tg}$ or $2.2 \mathrm{Pg}$ of carbon $\left(1\right.$ petagram $\left.=10^{15} \mathrm{gram}\right)$ in 1989 and then decreased slightly to $2103 \mathrm{Tg}$ or $2.1 \mathrm{Pg}$ of carbon in 1990 (Fig. 2 and App. D). The global net flux during the period 1850 to1990 was $124 \mathrm{Pg}$ of carbon. During this period, the greatest regional flux was from South and Southeast Asia (39 Pg of carbon), while the smallest regional flux was from North Africa and the Middle East (3 Pg of carbon). For the year 1990, the global total net flux was estimated to be $2.1 \mathrm{Pg}$ of carbon; for comparison, the estimated 1990 carbon flux to the atmosphere from fossil-fuel combustion and cement production has been estimated at 6.1 Pg of carbon (Marland et al. 1999).

This revised database provides estimates for all regions through 1990, whereas Houghton and Hackler (1995) provided estimates for only three regions (South and Central America, Tropical Africa, and South and Southeast Asia) through 1990, for one region (the Former Soviet Union) through 1985, and for the remaining five regions (North America, Europe, North Africa and the Middle East, China, and the Pacific Developed Region) through 1980. For some variables (e.g., fuelwood harvest in South and Southeast Asia, by forest type) the data extend beyond 1990.

The approach used in Houghton (1999) differs from that used in earlier estimates in several respects:

(1) The analysis for South and Southeast Asia has been reconstructed (Houghton and Hackler 1999) to directly assess the effects of logging based on mass of harvested material. This analysis is now methodologically consistent with that for other regions, whereas the approach used in Houghton and Hackler (1995) modeled the region based upon estimated degradation of forest biomass.

(2) The timber harvest rates for China (files chin-rat.* in Houghton and Hackler 1995, App. B in this document) were relabeled to correspond to the correct ecosystem. 


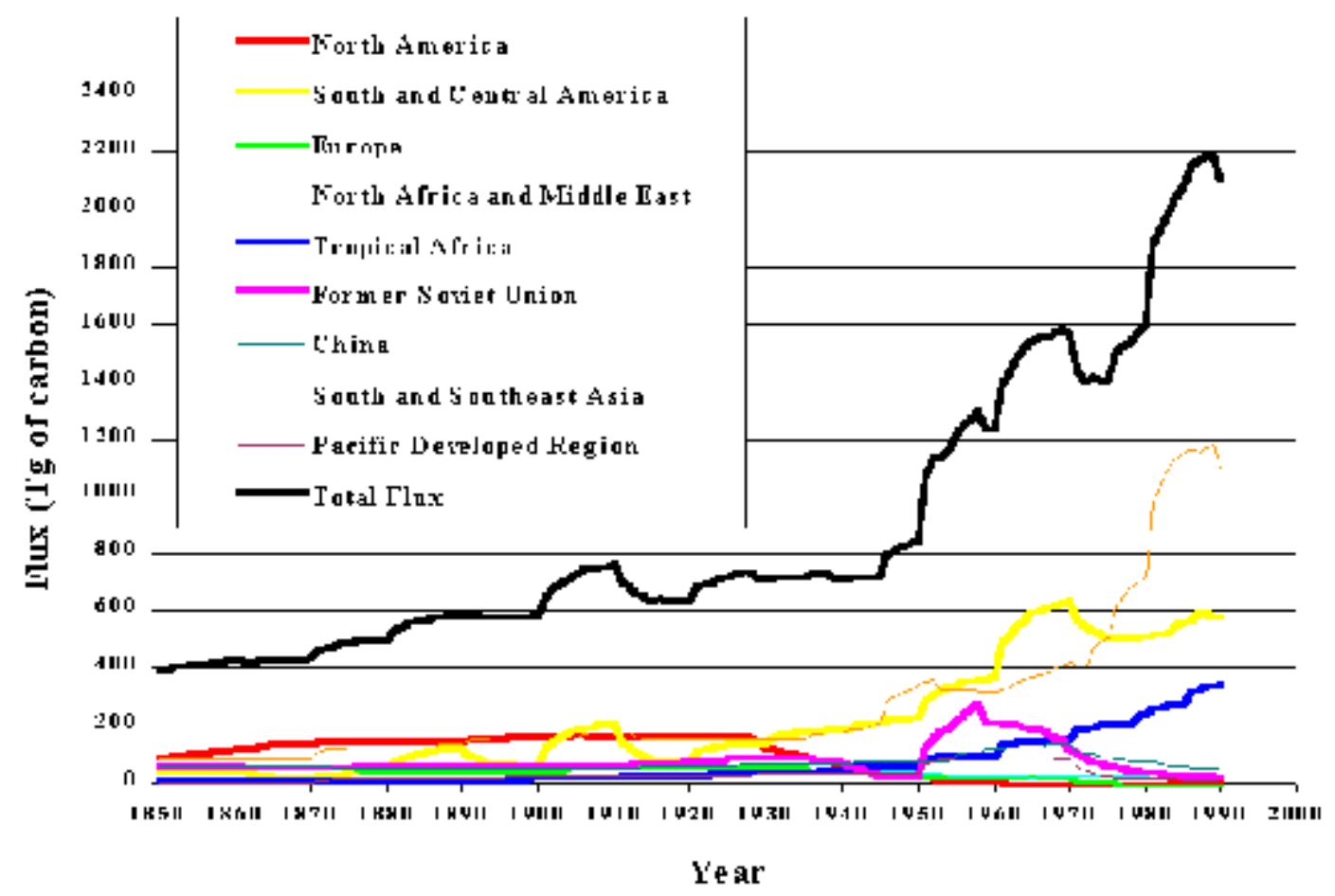

Figure 2. Net flux of carbon to the atmosphere from land-use changes, by region, 1850 to 1990.

(3) The clearing rates and harvest mass for the Former Soviet Union are documented in Melillo et al. (1988), replacing the input rates that had been taken from Houghton et al. (1983) and provided in Houghton and Hackler (1995) as files fsu-rat** (App. B in this document).

(4) Revised data for South and Central America (Houghton et al. 1991a, 1991b ) were used. The earlier data were provided in Houghton and Hackler (1995) as files scam-re.* and scam.rat.*.

(5) Houghton (1999) mentioned three other revisions to the earlier estimates: The residence time of plant debris removed during clearing for agriculture was reduced, forest plantations were considered, and deforestation rates were updated. 


\section{APPLICATIONS OF THE DATA}

This database will be useful for studies of the global carbon cycle, especially focusing on fluxes of carbon between terrestrial ecosystems and the atmosphere. The database will also be useful for studies of land-use change, agriculture, and forestry. The region- and ecosystem-specific parameters provided in Appendix B will be useful for estimating both the recovery of ecosystems following disturbance and the oxidation of carbon in wood products.

\section{DATA LIMITATIONS AND RESTRICTIONS}

The methodology of Houghton (1999) is limited to deliberate changes in land use (e.g., clearing for agriculture and harvest of forests for timber and fuelwood) and does not account for all processes that affect ecosystem carbon storage and fluxes (e.g., natural disturbances; fire suppression and silvicultural practices; and environmental factors, such as $\mathrm{CO}_{2}$, nitrogen deposition, acid precipitation, ultraviolet radiation, and climate, that affect vegetation). Furthermore, the analysis ignores fluxes of carbon to or from ecosystems not directly affected by land-use change. In a study of net flux from land-use change in the United States (Houghton et al. 1999), the authors concluded that such environmental factors as climate and increased $\mathrm{CO}_{2}$ could have accounted for 2 to 4 times as much carbon accumulation as did recovery from previous harvests.

Houghton and Hackler (1999) consider at length the uncertainties associated with estimates of net carbon flux from land-use change. For tropical Asia, they estimate the uncertainty of the long-term flux to be about $30 \%$. The sources of uncertainty are divided into estimating the areas of land affected by change, estimating the biomass of the land (especially in the years before human disturbance), and estimating changes in carbon stocks over time.

Houghton (1999) addresses the simplifications, approximations, and assumptions that are inherent in the estimation of carbon fluxes based on available data, such as estimation of time series of wood harvest or area of cropland by extrapolating from population time series and single-year per capita data.

Additional, region-specific, data limitations are mentioned in Houghton (1999):

(1) In China, Europe, North Africa and the Middle East, North America, the Pacific Developed Region, and South and Central America, harvest of timber was not distinguished from harvest of fuelwood, even though they have different carbon oxidation rates. 
(2) Only in South and Central America and in South and Southeast Asia was shifting cultivation considered.

(3) In the Former Soviet Union, the effects of grazing and peat drainage were not considered.

(4) In South and Central America, carbon flux from an increase in degraded lands was excluded.

(5) In South and Southeast Asia, the extraction of fuelwood during the early years may have been underestimated, because of an inverse relationship between per capita extraction and population density.

(6) In Tropical Africa, harvest of wood and shifting cultivation were not included.

The estimates of annual net carbon flux on a global total basis, derived from this database and reported by Houghton (1999), vary somewhat from estimates reported previously (e.g., Houghton et al. 1983, Houghton and Skole 1990, Houghton and Hackler 1995) [Fig. 3 and App. E; but note that the data for the period 1850 to 1859 attributed in Fig. 3 and App. E to Houghton et al. (1983) were not actually presented in that publication but are present in the data used in that publication]. According to the data presented in this numeric data package, the total flux over the period 1850 to 1980 was $103 \mathrm{Pg}$ of carbon (corrected from the $109 \mathrm{Pg}$ of carbon estimate reported in Houghton 1999). This estimate is considerably lower than that found in Houghton et al. (1983) for the (shorter) period 1860 to 1980 , $180 \mathrm{Pg}$ of carbon, which has been characterized (Houghton 1999) as an overestimate for three reasons: (1) The amount of soil carbon lost with cultivation was overestimated, because an observed $50 \%$ loss of carbon in the upper 20 to $30 \mathrm{~cm}$ of the soil column was applied to the top $1 \mathrm{~m}$ of soil; (2) estimates of forest biomass in Latin America and Africa were too high; and (3) there was no distinction between harvests of fuelwood and timber despite their very different efficiencies of wood removal. The estimate of global total net flux over the period 1850 to 1980 derived from the data in this numeric data package (103 Pg of carbon) is closer to the more recent estimate in Houghton and Skole (1990): A value of $110 \mathrm{Pg}$ of carbon is reported in that paper, although the authors (personal communication) have noted that the methodology and data described in the paper actually yield a total of $118 \mathrm{Pg}$ of carbon. It is also closer to the estimate of $106 \mathrm{Pg}$ of carbon in Houghton (1993) and to the estimate of $99 \mathrm{Pg}$ of carbon in Houghton and Hackler (1995).

\section{DATA CHECKS AND PROCESSING PERFORMED BY CDIAC}

An important part of the data-packaging process at CDIAC involves the quality assurance (QA) of data before distribution. To guarantee data of the highest possible quality, CDIAC performs extensive 
Houghton \& Hackler 2001, CDIAC NDP-050/R1 (http://cdiac.esd.ornl.gov/ndps/ndp050.html)

QA checks, examining the data for completeness, reasonableness, and accuracy, through close cooperation with the data contributor. 


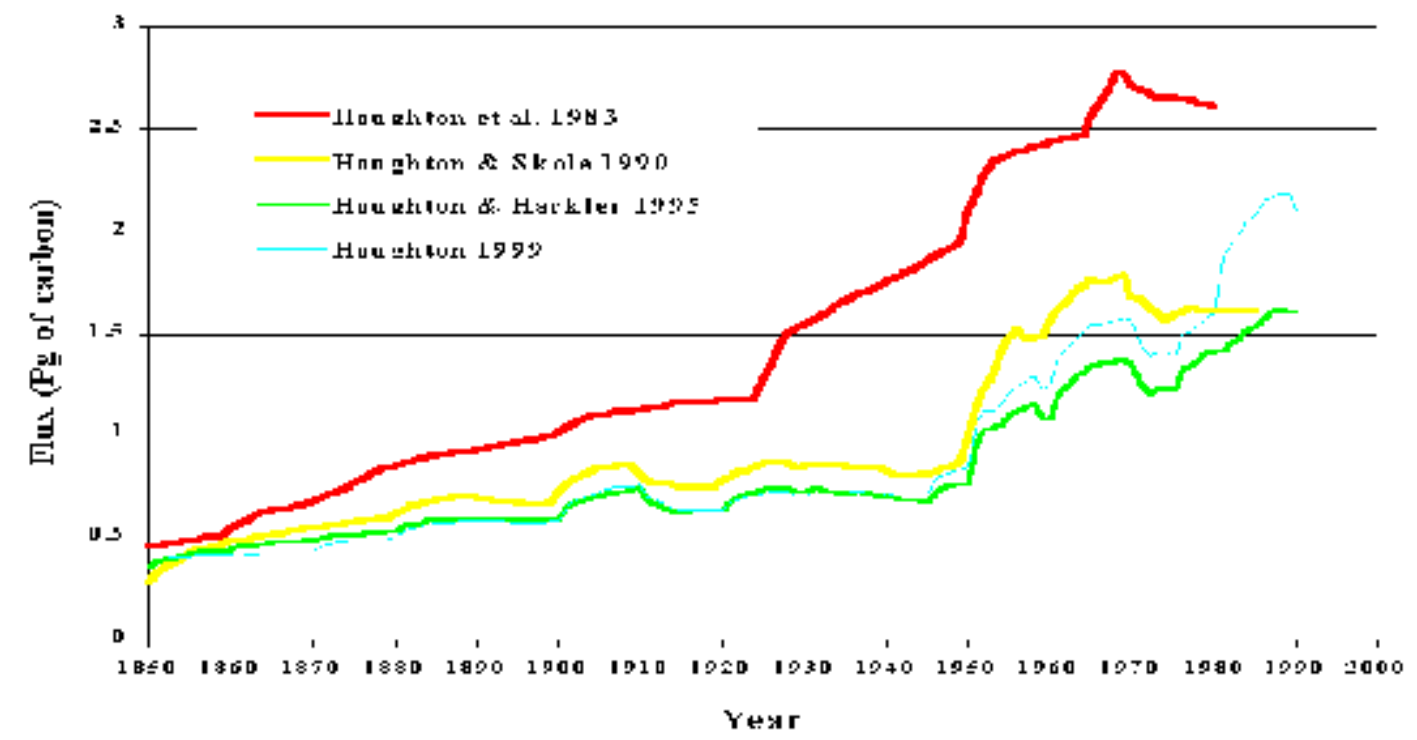

\section{Figure 3. Comparison of estimates of net flux of carbon to the atmosphere from land-use changes, 1850 to 1990.}

CDIAC did not attempt to run the bookkeeping model to validate the estimates presented in Houghton (1999). Rather, CDIAC focused its QA efforts on the format and consistency of the datasets and on comparing the values in the database with the corresponding values specified in Houghton (1999).

The annual net flux estimates by region for the period 1850 to 1990 were graphed and visually compared with Figure 5 in Houghton (1999), and the annual global total estimates for the period 1850 to 1990 were graphed and visually compared with Figure 6 in Houghton (1999).

The global total net flux estimates, derived from this database, for the periods 1850 to 1980 (103 Pg of carbon) and 1850 to 1990 (124 Pg of carbon) were compared with the corresponding totals reported in Table 3 of Houghton (1999). While the 1850 to 1990 estimates were identical, the 1850 to 1980 total derived from this database differed from the value of $109 \mathrm{Pg}$ of carbon reported in Houghton (1999). This was determined to be an error in Houghton (1999) rather than an error in the database. 
Regional total net flux estimates, derived from this database, for the period 1850 to 1990 and the average annual flux for the 1980s were compared with the corresponding totals reported in Table 2 of Houghton (1999). They all agreed, with the exception of the estimate of the 1850 to 1990 total for the Former Soviet Union, which is $10.7 \mathrm{Pg}$ of carbon according to this database but $10.4 \mathrm{Pg}$ of carbon according to Houghton (1999). This discrepancy is attributed to the current explicit specification of volume of timber and fuelwood harvest in the data input and modeling process (as opposed to the previous use of area harvested as a surrogate for the volume of harvest).

Equivalent files compare.dat and compare.wk1 list the year-by-year estimates of global total net flux plotted in Figure 6 of Houghton (1999), corresponding to the estimates presented in Houghton et al. (1983), Houghton and Skole (1990, as corrected), Houghton and Hackler (1995), and Houghton (1999, as corrected).

\section{INSTRUCTIONS FOR OBTAINING THE DATA AND DOCUMENTATION}

This database (NDP-050/R1) is available free of charge from CDIAC. The files are available via the Internet, from CDIAC's World Wide Web site (http://cdiac.esd.ornl.gov), or from CDIAC's anonymous file transfer protocol (FTP) area (cdiac.esd.ornl.gov) as follows:

1. $\quad$ FTP to cdiac.esd.ornl.gov (128.219.24.36).

2. Enter "ftp" as the user id.

3. Enter your electronic mail address as the password (e.g., fred@zulu.org).

4. Change to the directory "pub/ndp050" (i.e., use the command "cd pub/ndp050").

5. Set ftp to get ASCII files by using the ftp "ascii" command.

6. Retrieve the ASCII database documentation file by using the ftp "get ndp050.txt" command.

7. Retrieve the ASCII data files by using the ftp "mget *.dat" command.

8. Set ftp to get binary files by using the ftp "binary" command.

9. Retrieve the binary spreadsheet files by using the ftp "mget *.wk1" command.

10. Exit the system by using the ftp "quit" command.

11. Uncompress the files on your computer if they are obtained in compressed format. 
For non-Internet data acquisitions (e.g., diskette or CD-ROM) or for additional information, contact:

\author{
Information Services \\ Carbon Dioxide Information Analysis Center \\ Oak Ridge National Laboratory \\ P.O. Box 2008 \\ Oak Ridge, TN 37831-6335, U.S.A. \\ Telephone: 1-865-574-3645 \\ Telefax: 1-865-574-2232 \\ E-mail: cdiac@ornl.gov
}

\title{
6. REFERENCES
}

Brown, S., and G. Gaston. 1996. Tropical Africa: Land use, biomass, and carbon estimates for 1980 (R. C. Daniels, editor). ORNL/CDIAC-92, NDP-055. Carbon Dioxide Information Analysis Center, Oak Ridge National Laboratory, U.S. Department of Energy, Oak Ridge, Tennessee.

Houghton, R. A. 1993. The flux of carbon from changes in land use. pp. 39-42. In I. G. Enting and K. R. Lassey (eds.). Projections of Future $\mathrm{CO}_{2}$. Technical paper 27, CSIRO Division of Atmospheric Research, Mordialloc, Australia.

Houghton, R. A. 1999. The annual net flux of carbon to the atmosphere from changes in land use 1850-1990. Tellus 51B:298-313.

Houghton, R. A., R. D. Boone, J. R. Fruci, J. E. Hobbie, J. M. Melillo, C. A. Palm, B. J. Peterson, G. R. Shaver, G. M. Woodwell, B. Moore, D. L. Skole, and N. Myers. 1987. The flux of carbon from terrestrial ecosystems to the atmosphere in 1980 due to changes in land use: Geographic distribution of the global flux. Tellus 39B:122-139.

Houghton, R. A., and J. L. Hackler. 1995. Continental scale estimates of the biotic carbon flux from land cover change: 1850-1980 (R. C. Daniels, editor). ORNL/CDIAC-79, NDP-050. Carbon Dioxide Information Analysis Center, Oak Ridge National Laboratory, U.S. Department of Energy, Oak Ridge, Tennessee.

Houghton, R. A., and J. L. Hackler. 1999. Emissions of carbon from forestry and land-use change in tropical Asia. Global Change Biology 5:481-492. 
Houghton, R. A., J. L. Hackler, and K. T. Lawrence. 1999. The U.S. carbon budget: Contributions from land-use change. Science 285:574-578.

Houghton, R. A., J. E. Hobbie, J. M. Melillo, B. Moore, B. J. Peterson, G. R. Shaver, and G. M. Woodwell. 1983. Changes in the carbon content of terrestrial biota and soils between 1860 and 1980: A net release of $\mathrm{CO}_{2}$ to the atmosphere. Ecological Monographs 53:235-262.

Houghton, R. A., D. S. Lefkowitz, and D. L. Skole. 1991a. Changes in the landscape of Latin America between 1850 and 1980 (I). A progressive loss of forests. Forest Ecology Management 38:143-172.

Houghton, R. A., and D. L. Skole. 1990. Carbon. pp. 393-408. In B. L. Turner, W. C. Clark, R. W. Kates, J. F. Richards, J. T. Mathews, and W. B. Meyer (eds.), The Earth as Transformed by Human Action. Cambridge University Press, Cambridge, U.K.

Houghton, R. A., D. L. Skole, and D. S. Lefkowitz. 1991b. Changes in the landscape of Latin America between 1850 and 1980 (II). A net release of $\mathrm{CO}_{2}$ to the atmosphere. Forest Ecology Management 38:173-199.

Marland, G., A. Brenkert, and J. Olivier. 1999. $\mathrm{CO}_{2}$ from fossil fuel burning: A comparison of ORNL and EDGAR estimates of national emissions. Environmental Science \& Policy 2:265-273.

Melillo, J. M., J. R. Fruci, R. A. Houghton, B. Moore, and D. L. Skole. 1988. Land-use change in the Soviet Union between 1850 and 1980: Causes of a net release of $\mathrm{CO}_{2}$ to the atmosphere. Tellus 40B:116-128.

Olson, J. S., J. A. Watts, and L. J. Allison. 1985. Major world ecosystem complexes ranked by carbon in live vegetation: A database. NDP-017. Carbon Dioxide Information Analysis Center, Oak Ridge National Laboratory, U.S. Department of Energy, Oak Ridge, Tennessee.

Richards, J. F., and E. P. Flint. 1994. Historic land use and carbon estimates for South and Southeast Asia (R. C. Daniels, editor). ORNL/CDIAC-61, NDP-064. Carbon Dioxide Information Analysis Center, Oak Ridge National Laboratory, U.S. Department of Energy, Oak Ridge, Tennessee.

\section{LISTING OF FILES PROVIDED}


The database consists of five files (see Table 2), including this documentation file. The data files (ndp050.* and compare.*) are available in two formats: as flat ASCII files and as binary spreadsheet files (in Lotus $1-2-3^{\circledR}$ format, but readable by other spreadsheet programs).

\section{Table 2. Files in the database}

\begin{tabular}{rrrrr}
\hline $\begin{array}{c}\text { File } \\
\text { number }\end{array}$ & File name & $\begin{array}{c}\text { File } \\
\text { size } \\
(\mathrm{kB})\end{array}$ & File type & File description \\
\hline 1 & ndp050.txt & 137 & ASCII text & Documentation file \\
2 & ndp050.dat & 16 & ASCII text & Data file \\
3 & ndp050.wk1 & 26 & Binary spreadsheet & Data file \\
4 & compare.dat & 10 & ASCII text & Data file \\
5 & compare.wk1 & 13 & Binary spreadsheet & Data file \\
\hline
\end{tabular}

\section{DESCRIPTION OF THE DOCUMENTATION FILE}

The ndp050.txt (File 1) file is an ASCII text equivalent of this document.

\section{DESCRIPTION, FORMAT, AND PARTIAL LISTINGS OF THE ASCII DATA FILES}

Table 3 describes the format and contents of the ASCII data file ndp050.dat (File 2) distributed with this numeric data package. Table 3 also indicates the column in the corresponding spreadsheet file ndp050.wk1 in which each variable is found. There are no missing values in these two files. 
Table 3. Contents and format of ndp050.dat (File 2)

\begin{tabular}{|c|c|c|c|c|c|c|c|}
\hline Variable & $\begin{array}{l}\text { Variable } \\
\text { type }\end{array}$ & $\begin{array}{l}\text { Variable } \\
\text { width }\end{array}$ & $\begin{array}{l}\text { Starting } \\
\text { column }\end{array}$ & $\begin{array}{l}\text { Ending } \\
\text { column }\end{array}$ & Units & $\begin{array}{l}\text { Spreadsheet } \\
\text { column }\end{array}$ & $\begin{array}{l}\text { Definition and } \\
\text { comments }\end{array}$ \\
\hline YEAR & Integer & 4 & 5 & 8 & year & A & Year \\
\hline NAM & Real & 6 & 11 & 16 & $1000 \mathrm{Mg} \mathrm{C}$ & $\mathrm{B}$ & $\begin{array}{l}\text { Net flux for North } \\
\text { America }\end{array}$ \\
\hline SCAM & Real & 6 & 22 & 27 & $1000 \mathrm{Mg} \mathrm{C}$ & $\mathrm{C}$ & $\begin{array}{l}\text { Net flux for South } \\
\text { and Central } \\
\text { America }\end{array}$ \\
\hline EUROPE & Real & 6 & 31 & 36 & $1000 \mathrm{Mg} \mathrm{C}$ & $\mathrm{D}$ & $\begin{array}{l}\text { Net flux for } \\
\text { Europe }\end{array}$ \\
\hline NAFRME & Real & 5 & 45 & 49 & $1000 \mathrm{Mg} \mathrm{C}$ & $\mathrm{E}$ & $\begin{array}{l}\text { Net flux for North } \\
\text { Africa and the } \\
\text { Middle East }\end{array}$ \\
\hline TRAFR & Real & 6 & 54 & 59 & $1000 \mathrm{Mg} \mathrm{C}$ & $\mathrm{F}$ & $\begin{array}{l}\text { Net flux for } \\
\text { Tropical Africa }\end{array}$ \\
\hline FSU & Real & 6 & 64 & 69 & $1000 \mathrm{Mg} \mathrm{C}$ & $\mathrm{G}$ & $\begin{array}{l}\text { Net flux for the } \\
\text { Former Soviet } \\
\text { Union }\end{array}$ \\
\hline CHINA & Real & 6 & 74 & 79 & $1000 \mathrm{Mg} \mathrm{C}$ & $\mathrm{H}$ & Net flux for China \\
\hline SSEASIA & Real & 7 & 84 & 90 & $1000 \mathrm{Mg} \mathrm{C}$ & I & $\begin{array}{l}\text { Net flux for South } \\
\text { and Southeast } \\
\text { Asia }\end{array}$ \\
\hline PACDR & Real & 5 & 97 & 101 & $1000 \mathrm{Mg} \mathrm{C}$ & $\mathrm{J}$ & $\begin{array}{l}\text { Net flux for the } \\
\text { Pacific Developed } \\
\text { Region }\end{array}$ \\
\hline TOTAL & Real & 8 & 104 & 111 & $1000 \mathrm{Mg} \mathrm{C}$ & $\mathrm{K}$ & Net global flux \\
\hline
\end{tabular}

First two data records:

\begin{tabular}{|c|c|c|c|c|c|c|c|c|c|c|}
\hline 1850 & 87.28 & 42.48 & 55.04 & 3.98 & 5.61 & 58.56 & 56.52 & 85.63 & 2.05 & 397.145 \\
\hline 1851 & 87.22 & 42.18 & 55.02 & 3.98 & 6.47 & 58.55 & 56.50 & 85.20 & 2.04 & 397.164 \\
\hline
\end{tabular}

Last two data records:

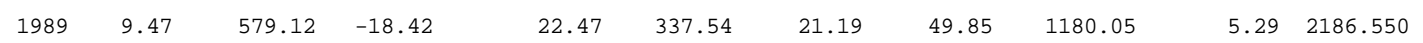




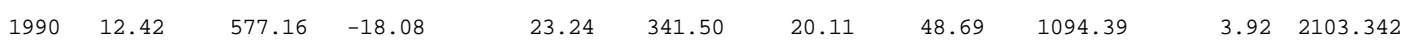

Table 4 describes the format and contents of the ASCII data file compare.dat (File 4) distributed with this numeric data package. Table 4 also indicates the column in the corresponding spreadsheet file compare.wk1 in which each variable is found. The missing-value indicator in the ascii file is ! 9.999 (in the spreadsheet file, cells representing missing values are simply left blank).

Table 4. Contents and format of compare.dat (File 4)

\begin{tabular}{|c|c|c|c|c|c|c|c|}
\hline Variable & $\begin{array}{l}\text { Variable } \\
\text { type }\end{array}$ & $\begin{array}{l}\text { Variable } \\
\text { width }\end{array}$ & $\begin{array}{l}\text { Starting } \\
\text { column }\end{array}$ & $\begin{array}{l}\text { Ending } \\
\text { column }\end{array}$ & Units & $\begin{array}{l}\text { Spreadsheet } \\
\text { column }\end{array}$ & $\begin{array}{l}\text { Definition and } \\
\text { comments }\end{array}$ \\
\hline YEAR & Integer & 4 & 1 & 4 & year & A & Year \\
\hline HETAL83 & Real & 6 & 13 & 18 & $1000 \mathrm{Mg} \mathrm{C}$ & B & $\begin{array}{r}\text { Global total net } \\
\text { flux, from } \\
\text { Houghton et al. } \\
\text { (1983) }\end{array}$ \\
\hline HS90 & Real & 6 & 31 & 36 & $1000 \mathrm{Mg} \mathrm{C}$ & $\mathrm{C}$ & $\begin{array}{r}\text { Global total net } \\
\text { flux, from } \\
\text { Houghton and } \\
\text { Skole (1990), as } \\
\text { corrected }\end{array}$ \\
\hline HH95 & Real & 5 & 51 & 55 & $1000 \mathrm{Mg} \mathrm{C}$ & D & $\begin{array}{r}\text { Global total net } \\
\text { flux, from } \\
\text { Houghton and } \\
\text { Hackler (1995) }\end{array}$ \\
\hline H99 & Real & 5 & 63 & 67 & $1000 \mathrm{Mg} \mathrm{C}$ & E & $\begin{array}{r}\text { Global total net } \\
\text { flux, from } \\
\text { Houghton (1999) }\end{array}$ \\
\hline
\end{tabular}

First two data records:
1850
0.458
0.278
0.352
0.397
1851
0.464
0.319
0.383
0.397

Last two data records:

$\begin{array}{lllll}1989 & -9.999 & -9.999 & 1.611 & 2.187 \\ 1990 & -9.999 & -9.999 & 1.614 & 2.103\end{array}$




\section{DESCRIPTION AND FORMAT OF THE LOTUS 1-2-3 ${ }^{\circledR}$ BINARY SPREADSHEET FILES}

Lotus $1-2-3^{\circledR}$ binary spreadsheet file ndp050.wk1 (File 3) contains the same information as the corresponding ASCII file ndp050.dat (File 2), and Lotus 1-2-3 ${ }^{\circledR}$ binary spreadsheet file compare.wk1 (File 5) contains the same information as the corresponding ASCII file compare.dat (File 4)

Table 3, which describes the contents and format of ndp050.dat, also indicates the column of ndp050.wk1 in which each variable is found, and Table 4, which describes the contents and format of compare.dat, also indicates the column of compare.wk1 in which each variable is found.

\section{SAS ${ }^{\circledR}$ AND FORTRAN CODES TO ACCESS THE DATA}

The following is $\mathrm{SAS}^{\circledR}$ code to read file ndp050.dat.

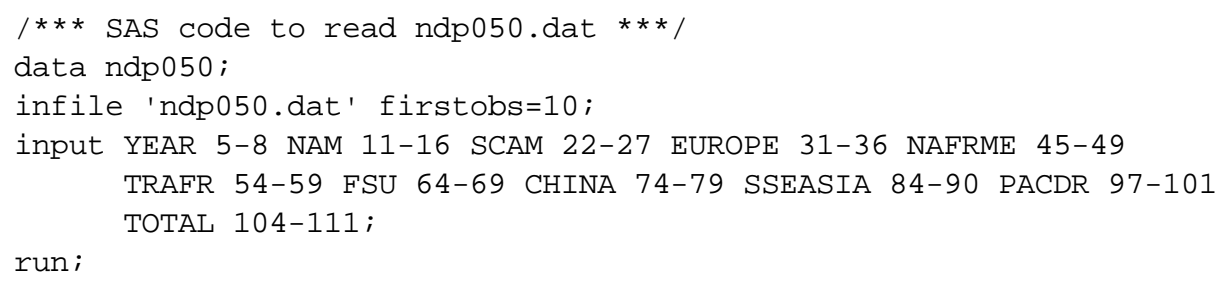


The following is Fortran code to read file ndp050.dat.

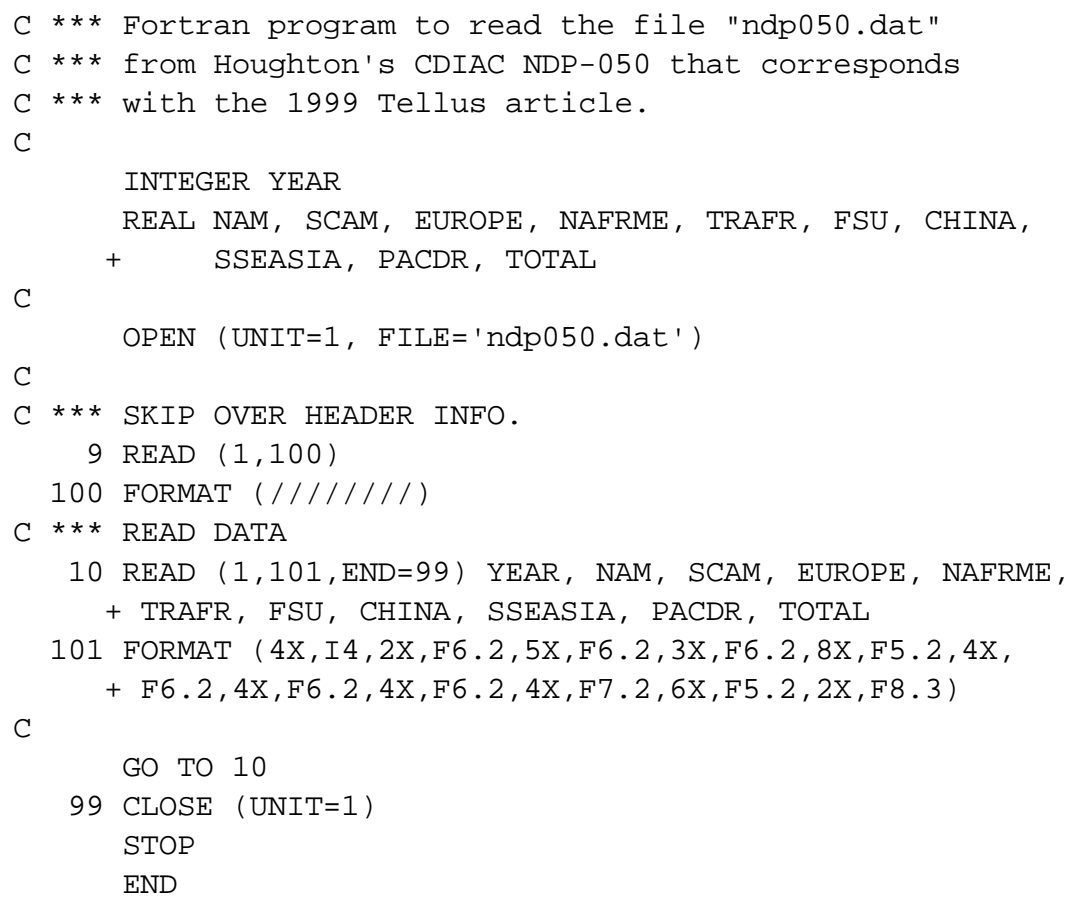

The following is $\mathrm{SAS}^{\circledR}$ code to read file compare.dat.

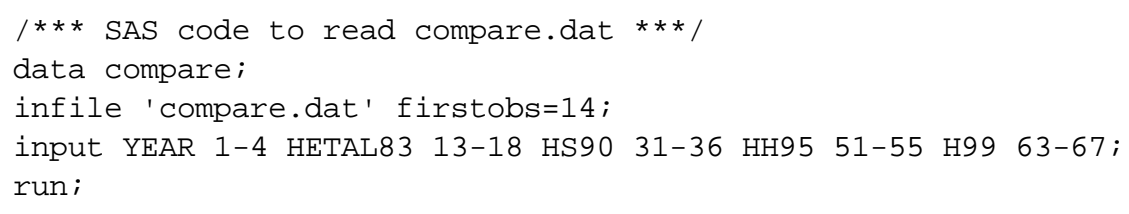

The following is Fortran code to read file compare.dat.

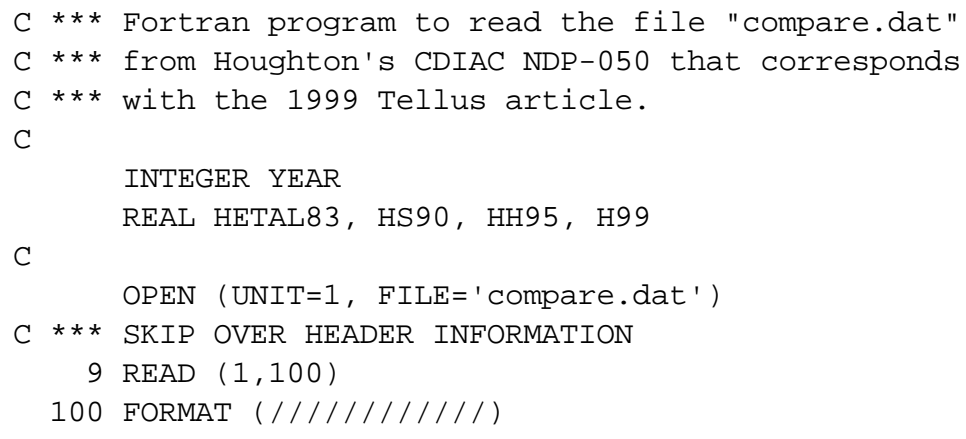


Houghton \& Hackler 2001, CDIAC NDP-050/R1 (http://cdiac.esd.ornl.gov/ndps/ndp050.html)

$\mathrm{C} \star \star \star$ READ DATA

10 READ (1, 101, END=99) YEAR, HETAL83, HS90, HH95, H99

101 FORMAT (I 4, 8X, F6.3, 12X,F6.3, 14X, F5.3, 7X, F5.3)

$\mathrm{C}$

GO TO 10

99 CLOSE (UNIT=1)

STOP

END 


\section{APPENDIX A. ECOSYSTEM AREA BY REGION}

This listing indicates the area (in units of $10^{6}$ hectare) in different ecosystems for the nine regions in this database, for the years 1700, 1850, and 1990, along with the percent change from 1850 to 1990 . The values in this listing replace the values in files areas.* in Houghton and Hackler (1995), the previous version of this database. Missing values are denoted by ! 9999.

\begin{tabular}{|c|c|c|c|c|c|}
\hline & & 1700 & 1850 & 1990 & $\begin{array}{r}\text { \% Change } \\
1850-1990\end{array}$ \\
\hline \multicolumn{6}{|c|}{ North America } \\
\hline & Temperate evergreen forest & 236 & 222 & 215 & -0.03 \\
\hline & Temperate deciduous forest & 157 & 125 & 118 & -0.06 \\
\hline & Boreal forest & 325 & 325 & 322 & -0.01 \\
\hline & Temperate woodland/shrubland & 302 & 302 & 292 & -0.03 \\
\hline & Temperate grassland & 568 & 481 & 172 & -0.64 \\
\hline \multicolumn{6}{|c|}{ South and Central America } \\
\hline & Tropical evergreen forest & -9999 & 310 & 299 & -0.03 \\
\hline & Tropical seasonal forest & -9999 & 756 & 604 & -0.20 \\
\hline & Tropical open forest & -9999 & 427 & 287 & -0.33 \\
\hline & Temperate evergreen forest & -9999 & 67 & 57 & -0.15 \\
\hline & Temperate seasonal forest & -9999 & 58 & 56 & -0.03 \\
\hline \multicolumn{6}{|l|}{ Europe } \\
\hline & Temperate evergreen forest & 85 & 66 & 72 & 0.10 \\
\hline & Temperate deciduous forest & 65 & 56 & 56 & -0.01 \\
\hline & Boreal forest & 35 & 28 & 27 & 0.00 \\
\hline & Temperate woodland/shrubland & 45 & 45 & 45 & 0.00 \\
\hline & Temperate grassland & 88 & 42 & 27 & -0.36 \\
\hline \multicolumn{6}{|c|}{ North Africa and the Middle East } \\
\hline & Temperate evergreen forest & 17.7 & 16 & 7 & -0.56 \\
\hline & Tropical moist forest & 2.1 & 2 & 2 & 0.00 \\
\hline & Tropical grassland & 90 & 86 & 44 & -0.49 \\
\hline & Desert scrub & 979 & 961 & 793 & -0.17 \\
\hline & Tropical woodland/shrubland & 32.4 & 31 & 19 & -0.40 \\
\hline \multirow[t]{3}{*}{ Tropical } & Africa & & & & \\
\hline & Closed forest & -9999 & 605 & 553 & -0.09 \\
\hline & Open forest & -9999 & 198 & 105 & -0.47 \\
\hline \multicolumn{6}{|l|}{ Former } \\
\hline & Temperate evergreen forest & 88 & 88 & 88 & 0.00 \\
\hline & Temperate deciduous forest & 121 & 78 & 54 & -0.31 \\
\hline & Boreal forest & 613 & 613 & 613 & 0.00 \\
\hline & Temperate woodland/shrubland & 186 & 186 & 186 & 0.00 \\
\hline & Temperate grassland & 175 & 144 & 31 & -0.78 \\
\hline
\end{tabular}




\begin{tabular}{|c|c|c|c|c|c|}
\hline & & 1700 & 1850 & 1990 & $\begin{array}{r}\circ \text { Change } \\
1850-1990\end{array}$ \\
\hline \multicolumn{6}{|l|}{ China } \\
\hline & Temperate evergreen forest & 122 & 49 & 82 & 0.66 \\
\hline & Temperate deciduous forest & 103 & 76 & 48 & -0.38 \\
\hline & Temperate grassland & 723 & 586 & 439 & -0.25 \\
\hline & Tropical moist forest & 18 & 14 & 8 & -0.43 \\
\hline \multicolumn{6}{|c|}{ South and Southeast Asia } \\
\hline & Tropical moist forest & -9999 & 177 & 94 & -0.47 \\
\hline & Tropical seasonal forest & -9999 & 179 & 89 & -0.50 \\
\hline & Tropical open forest & -9999 & 52 & 40 & -0.23 \\
\hline & Tropical grassland & -9999 & 55 & 36 & -0.35 \\
\hline \multicolumn{6}{|l|}{ Pacific D } \\
\hline & Temperate evergreen forest & 14 & 14 & 14 & 0.00 \\
\hline & Temperate deciduous forest & 14 & 14 & 14 & 0.00 \\
\hline & Tropical moist forest & 72 & 72 & 64 & -0.12 \\
\hline & Tropical grassland & 497 & 496 & 70 & -0.86 \\
\hline & Tropical woodland/shrubland & 120 & 120 & 106 & -0.11 \\
\hline
\end{tabular}




\section{APPENDIX B. REGIONAL LAND-USE CHANGE AND WOOD HARVEST DATA}

The following listing provides the regional details of fuelwood (nonindustrial logging) and timber (industrial logging) harvest by forest type, changes in area of pasture, forest plantation, afforestation, forest clearing for croplands, and lands in shifting cultivation. The values in this listing replace the values in the indicated ascii and binary spreadsheet files in Houghton and Hackler (1995), the previous version of this database.

\section{South and Southeast Asia}

The following values replace the data in files asia-rat.* in Houghton and Hackler (1995).

South and Southeast Asia - Fuelwood Harvest $\left(10^{6} \mathrm{Mg} \mathrm{C}\right.$ per year):

\begin{tabular}{|c|c|c|c|c|c|c|c|}
\hline & ropical & Tropical & Tropical & 1776 & 8.58 & 10.50 & 4.50 \\
\hline & moist & seasonal & open & 1777 & 8.58 & 10.50 & 4.50 \\
\hline Year & forest & forest & forest & 1778 & 8.58 & 10.50 & 4.50 \\
\hline & & & & 1779 & 8.58 & 10.50 & 4.50 \\
\hline 1751 & 8.58 & 10.50 & 4.50 & 1780 & 8.58 & 10.50 & 4.50 \\
\hline 1752 & 8.58 & 10.50 & 4.50 & & Tropical & Tropical & Tropical \\
\hline 1753 & 8.58 & 10.50 & 4.50 & & moist & seasonal & open \\
\hline 1754 & 8.58 & 10.50 & 4.50 & Year & forest & forest & forest \\
\hline 1755 & 8.58 & 10.50 & 4.50 & & & & \\
\hline 1756 & 8.58 & 10.50 & 4.50 & 1781 & 8.58 & 10.50 & 4.50 \\
\hline 1757 & 8.58 & 10.50 & 4.50 & 1782 & 8.58 & 10.50 & 4.50 \\
\hline 1758 & 8.58 & 10.50 & 4.50 & 1783 & 8.58 & 10.50 & 4.50 \\
\hline 1759 & 8.58 & 10.50 & 4.50 & 1784 & 8.58 & 10.50 & 4.50 \\
\hline 1760 & 8.58 & 10.50 & 4.50 & 1785 & 8.58 & 10.50 & 4.50 \\
\hline 1761 & 8.58 & 10.50 & 4.50 & 1786 & 8.58 & 10.50 & 4.50 \\
\hline 1762 & 8.58 & 10.50 & 4.50 & 1787 & 8.58 & 10.50 & 4.50 \\
\hline 1763 & 8.58 & 10.50 & 4.50 & 1788 & 8.58 & 10.50 & 4.50 \\
\hline 1764 & 8.58 & 10.50 & 4.50 & 1789 & 8.58 & 10.50 & 4.50 \\
\hline 1765 & 8.58 & 10.50 & 4.50 & 1790 & 8.58 & 10.50 & 4.50 \\
\hline 1766 & 8.58 & 10.50 & 4.50 & 1791 & 8.58 & 10.50 & 4.50 \\
\hline 1767 & 8.58 & 10.50 & 4.50 & 1792 & 8.58 & 10.50 & 4.50 \\
\hline 1768 & 8.58 & 10.50 & 4.50 & 1793 & 8.58 & 10.50 & 4.50 \\
\hline 1769 & 8.58 & 10.50 & 4.50 & 1794 & 8.58 & 10.50 & 4.50 \\
\hline 1770 & 8.58 & 10.50 & 4.50 & 1795 & 8.58 & 10.50 & 4.50 \\
\hline 1771 & 8.58 & 10.50 & 4.50 & 1796 & 8.58 & 10.50 & 4.50 \\
\hline 1772 & 8.58 & 10.50 & 4.50 & 1797 & 8.58 & 10.50 & 4.50 \\
\hline 1773 & 8.58 & 10.50 & 4.50 & 1798 & 8.58 & 10.50 & 4.50 \\
\hline 1774 & 8.58 & 10.50 & 4.50 & 1799 & 8.58 & 10.50 & 4.50 \\
\hline 1775 & 8.58 & 10.50 & 4.50 & 1800 & 8.58 & 10.50 & 4.50 \\
\hline
\end{tabular}




$\begin{array}{llll}1801 & 8.58 & 10.50 & 4.50 \\ 1802 & 8.62 & 10.55 & 4.52 \\ 1803 & 8.66 & 10.60 & 4.54 \\ 1804 & 8.70 & 10.65 & 4.56 \\ 1805 & 8.74 & 10.70 & 4.59 \\ 1806 & 8.78 & 10.75 & 4.61 \\ 1807 & 8.82 & 10.80 & 4.63 \\ 1808 & 8.86 & 10.85 & 4.65 \\ 1809 & 8.90 & 10.90 & 4.67 \\ 1810 & 8.94 & 10.95 & 4.69\end{array}$

South and Southeast Asia — Fuelwood Harvest (continued)

\begin{tabular}{|c|c|c|c|c|c|c|c|}
\hline & ropical & Tropical & Tropical & 1842 & 10.25 & 12.55 & 5.38 \\
\hline & moist & seasonal & open & 1843 & 10.29 & 12.60 & 5.40 \\
\hline Year & forest & forest & forest & 1844 & 10.33 & 12.65 & 5.42 \\
\hline & & & & 1845 & 10.37 & 12.70 & 5.44 \\
\hline 1811 & 8.98 & 11.00 & 4.71 & 1846 & 10.41 & 12.75 & 5.46 \\
\hline 1812 & 9.03 & 11.05 & 4.74 & 1847 & 10.45 & 12.80 & 5.49 \\
\hline 1813 & 9.07 & 11.10 & 4.76 & 1848 & 10.50 & 12.85 & 5.51 \\
\hline 1814 & 9.11 & 11.15 & 4.78 & 1849 & 10.54 & 12.90 & 5.53 \\
\hline 1815 & 9.15 & 11.20 & 4.80 & 1850 & 10.58 & 12.95 & 5.55 \\
\hline 1816 & 9.19 & 11.25 & 4.82 & 1851 & 10.66 & 13.05 & 5.59 \\
\hline 1817 & 9.23 & 11.30 & 4.84 & 1852 & 10.74 & 13.15 & 5.63 \\
\hline 1818 & 9.27 & 11.35 & 4.86 & 1853 & 10.82 & 13.24 & 5.68 \\
\hline 1819 & 9.31 & 11.40 & 4.89 & 1854 & 10.90 & 13.34 & 5.72 \\
\hline 1820 & 9.35 & 11.45 & 4.91 & 1855 & 10.98 & 13.44 & 5.76 \\
\hline 1821 & 9.39 & 11.50 & 4.93 & 1856 & 11.06 & 13.54 & 5.80 \\
\hline 1822 & 9.43 & 11.55 & 4.95 & 1857 & 11.14 & 13.64 & 5.84 \\
\hline 1823 & 9.47 & 11.60 & 4.97 & & Tropical & Tropical & Tropical \\
\hline 1824 & 9.52 & 11.65 & 4.99 & & moist & seasonal & open \\
\hline 1825 & 9.56 & 11.70 & 5.01 & Year & forest & forest & forest \\
\hline 1826 & 9.60 & 11.75 & 5.04 & & & & \\
\hline 1827 & 9.64 & 11.80 & 5.06 & 1858 & 11.22 & 13.73 & 5.89 \\
\hline 1828 & 9.68 & 11.85 & 5.08 & 1859 & 11.30 & 13.83 & 5.93 \\
\hline 1829 & 9.72 & 11.90 & 5.10 & 1860 & 11.38 & 13.93 & 5.97 \\
\hline 1830 & 9.76 & 11.95 & 5.12 & 1861 & 11.46 & 14.03 & 6.01 \\
\hline 1831 & 9.80 & 12.00 & 5.14 & 1862 & 11.54 & 14.13 & 6.05 \\
\hline 1832 & 9.84 & 12.05 & 5.16 & 1863 & 11.62 & 14.22 & 6.10 \\
\hline 1833 & 9.88 & 12.10 & 5.19 & 1864 & 11.70 & 14.32 & 6.14 \\
\hline 1834 & 9.92 & 12.15 & 5.21 & 1865 & 11.78 & 14.42 & 6.18 \\
\hline 1835 & 9.96 & 12.20 & 5.23 & 1866 & 11.86 & 14.52 & 6.22 \\
\hline 1836 & 10.01 & 12.25 & 5.25 & 1867 & 11.94 & 14.62 & 6.26 \\
\hline 1837 & 10.05 & 12.30 & 5.27 & 1868 & 12.02 & 14.71 & 6.31 \\
\hline 1838 & 10.09 & 12.35 & 5.29 & 1869 & 12.10 & 14.81 & 6.35 \\
\hline 1839 & 10.13 & 12.40 & 5.31 & 1870 & 12.18 & 14.91 & 6.39 \\
\hline 1840 & 10.17 & 12.45 & 5.34 & 1871 & 12.26 & 15.01 & 6.43 \\
\hline 1841 & 10.21 & 12.50 & 5.36 & 1872 & 12.34 & 15.11 & 6.47 \\
\hline
\end{tabular}




$\begin{array}{llll}1873 & 12.42 & 15.20 & 6.52 \\ 1874 & 12.50 & 15.30 & 6.56 \\ 1875 & 12.58 & 15.40 & 6.60 \\ 1876 & 12.66 & 15.50 & 6.64 \\ 1877 & 12.74 & 15.60 & 6.68 \\ 1878 & 12.82 & 15.69 & 6.73 \\ 1879 & 12.90 & 15.79 & 6.77 \\ 1880 & 12.98 & 15.89 & 6.81 \\ 1881 & 13.06 & 15.99 & 6.85 \\ 1882 & 13.14 & 16.09 & 6.89 \\ 1883 & 13.22 & 16.18 & 6.94 \\ 1884 & 13.30 & 16.28 & 6.98 \\ 1885 & 13.38 & 16.38 & 7.02 \\ 1886 & 13.46 & 16.48 & 7.06 \\ 1887 & 13.54 & 16.58 & 7.10 \\ 1888 & 13.62 & 16.67 & 7.15 \\ 1889 & 13.70 & 16.77 & 7.19 \\ 1890 & 13.78 & 16.87 & 7.23 \\ 1891 & 13.86 & 16.97 & 7.27 \\ 1892 & 13.94 & 17.07 & 7.31 \\ 1893 & 14.02 & 17.16 & 7.36 \\ 1894 & 14.10 & 17.26 & 7.40 \\ 1895 & 14.18 & 17.36 & 7.44 \\ 1896 & 14.26 & 17.46 & 7.48 \\ 1897 & 14.34 & 17.56 & 7.52 \\ 1898 & 14.42 & 17.65 & 7.57 \\ 1899 & 14.50 & 17.75 & 7.61 \\ 1900 & 14.58 & 17.85 & 7.65 \\ 1901 & 14.69 & 17.99 & 7.71 \\ 1902 & 14.81 & 18.13 & 7.77 \\ 1903 & 14.92 & 18.27 & 7.83 \\ 1904 & 15.04 & 18.41 & 7.89\end{array}$

South and Southeast Asia — Fuelwood Harvest (continued)

\begin{tabular}{rrrrrrrr}
\multicolumn{2}{r}{$\begin{array}{r}\text { Tropical } \\
\text { moist }\end{array}$} & $\begin{array}{r}\text { Tropical } \\
\text { seasonal } \\
\text { forest }\end{array}$ & $\begin{array}{r}\text { Tropical } \\
\text { open } \\
\text { forest }\end{array}$ & 1914 & 16.18 & 19.81 & 8.49 \\
Year & & & 1915 & 16.29 & 19.95 & 8.55 \\
1905 & 15.15 & 18.55 & 7.95 & 1917 & 16.41 & 20.09 & 8.61 \\
1906 & 15.27 & 18.69 & 8.01 & 1918 & 16.64 & 20.23 & 8.67 \\
1907 & 15.38 & 18.83 & 8.07 & 1919 & 16.75 & 20.51 & 8.73 \\
1908 & 15.49 & 18.97 & 8.13 & 1920 & 16.87 & 20.65 & 8.85 \\
1909 & 15.61 & 19.11 & 8.19 & 1921 & 16.98 & 20.79 & 8.91 \\
1910 & 15.72 & 19.25 & 8.25 & 1922 & 17.10 & 20.93 & 8.97 \\
1911 & 15.84 & 19.39 & 8.31 & 1923 & 17.21 & 21.07 & 9.03 \\
1912 & 15.95 & 19.53 & 8.37 & 1924 & 17.32 & 21.21 & 9.09 \\
1913 & 16.07 & 19.67 & 8.43 & 1925 & 17.44 & 21.35 & 9.15 \\
& & & & 1926 & 17.71 & 21.69 & 9.29
\end{tabular}


Houghton \& Hackler 2001, CDIAC NDP-050/R1 (http://cdiac.esd.ornl.gov/ndps/ndp050.html)

\begin{tabular}{|c|c|c|c|c|c|c|c|}
\hline 1927 & 17.99 & 22.02 & 9.44 & 1971 & 41.75 & 48.86 & 20.94 \\
\hline 1928 & 18.26 & 22.36 & 9.58 & 1972 & 42.92 & 49.77 & 21.33 \\
\hline 1929 & 18.54 & 22.69 & 9.73 & 1973 & 43.94 & 50.68 & 21.72 \\
\hline 1930 & 18.81 & 23.03 & 9.87 & 1974 & 45.51 & 51.59 & 22.11 \\
\hline 1931 & 19.08 & 23.37 & 10.01 & 1975 & 47.22 & 52.50 & 22.50 \\
\hline 1932 & 19.36 & 23.70 & 10.16 & 1976 & 48.17 & 53.76 & 23.04 \\
\hline 1933 & 19.63 & 24.04 & 10.30 & 1977 & 49.03 & 55.02 & 23.58 \\
\hline 1934 & 19.91 & 24.37 & 10.45 & 1978 & 49.89 & 56.28 & 24.12 \\
\hline 1935 & 20.18 & 24.71 & 10.59 & 1979 & 50.76 & 57.54 & 24.66 \\
\hline 1936 & 20.46 & 25.05 & 10.73 & 1980 & 51.82 & 58.80 & 25.20 \\
\hline 1937 & 20.73 & 25.38 & 10.88 & 1981 & 53.04 & 60.13 & 25.77 \\
\hline 1938 & 21.01 & 25.72 & 11.02 & 1982 & 54.11 & 61.46 & 26.34 \\
\hline 1939 & 21.28 & 26.05 & 11.17 & 1983 & 55.23 & 62.79 & 26.91 \\
\hline 1940 & 21.55 & 26.39 & 11.31 & 1984 & 56.46 & 64.12 & 27.48 \\
\hline 1941 & 21.83 & 26.73 & 11.45 & 1985 & 57.79 & 65.45 & 28.05 \\
\hline 1942 & 22.10 & 27.06 & 11.60 & 1986 & 58.93 & 66.78 & 28.62 \\
\hline 1943 & 22.38 & 27.40 & 11.74 & 1987 & 59.95 & 68.11 & 29.19 \\
\hline 1944 & 22.65 & 27.73 & 11.89 & 1988 & 61.04 & 69.44 & 29.76 \\
\hline 1945 & 22.93 & 28.07 & 12.03 & 1989 & 62.21 & 70.77 & 30.33 \\
\hline 1946 & 23.20 & 28.41 & 12.17 & 1990 & 63.59 & 72.10 & 30.90 \\
\hline 1947 & 23.48 & 28.74 & 12.32 & 1991 & 64.61 & 72.10 & 30.90 \\
\hline 1948 & 23.75 & 29.08 & 12.46 & 1992 & 67.58 & 72.10 & 30.90 \\
\hline 1949 & 24.02 & 29.41 & 12.61 & 1993 & 70.94 & 72.10 & 30.90 \\
\hline 1950 & 24.30 & 29.75 & 12.75 & 1994 & 74.01 & 72.10 & 30.90 \\
\hline 1951 & 25.04 & 30.66 & 13.14 & 1995 & 77.04 & 72.10 & 30.90 \\
\hline \multicolumn{2}{|c|}{$\begin{array}{r}\text { Tropical } \\
\text { moist }\end{array}$} & $\begin{array}{l}\text { Tropical } \\
\text { seasonal }\end{array}$ & $\begin{array}{r}\text { Tropical } \\
\text { open }\end{array}$ & & & & \\
\hline Year & forest & forest & forest & & & & \\
\hline 1952 & 25.79 & 31.57 & 13.53 & & & & \\
\hline 1953 & 26.53 & 32.48 & 13.92 & & & & \\
\hline 1954 & 27.27 & 33.39 & 14.31 & & & & \\
\hline 1955 & 28.02 & 34.30 & 14.70 & & & & \\
\hline 1956 & 28.76 & 35.21 & 15.09 & & & & \\
\hline 1957 & 29.50 & 36.12 & 15.48 & & & & \\
\hline 1958 & 30.25 & 37.03 & 15.87 & & & & \\
\hline 1959 & 30.99 & 37.94 & 16.26 & & & & \\
\hline 1960 & 31.73 & 38.85 & 16.65 & & & & \\
\hline 1961 & 32.47 & 39.76 & 17.04 & & & & \\
\hline 1962 & 33.17 & 40.67 & 17.43 & & & & \\
\hline 1963 & 33.86 & 41.58 & 17.82 & & & & \\
\hline 1964 & 34.63 & 42.49 & 18.21 & & & & \\
\hline 1965 & 35.48 & 43.40 & 18.60 & & & & \\
\hline 1966 & 36.32 & 44.31 & 18.99 & & & & \\
\hline 1967 & 37.29 & 45.22 & 19.38 & & & & \\
\hline 1968 & 38.31 & 46.13 & 19.77 & & & & \\
\hline 1969 & 39.41 & 47.04 & 20.16 & & & & \\
\hline 1970 & 40.50 & 47.95 & 20.55 & & & & \\
\hline
\end{tabular}


South and Southeast Asia — Timber Harvest $\left(10^{6} \mathrm{Mg} \mathrm{C}\right.$ per year):

\begin{tabular}{|c|c|c|c|c|c|c|c|}
\hline & ropical & Tropical & Tropi & 1792 & 1.49 & 0.88 & 0.00 \\
\hline & moist & seasonal & open & 1793 & 1.49 & 0.89 & 0.00 \\
\hline Year & forest & forest & forest & 1794 & 1.50 & 0.89 & 0.00 \\
\hline & & & & 1795 & 1.51 & 0.89 & 0.00 \\
\hline 1750 & 1.26 & 0.80 & 0.00 & 1796 & 1.51 & 0.89 & 0.00 \\
\hline 1751 & 1.27 & 0.80 & 0.00 & & Tropical & Tropical & Tropical \\
\hline 1752 & 1.27 & 0.80 & 0.00 & & moist & seasonal & open \\
\hline 1753 & 1.28 & 0.81 & 0.00 & Year & forest & forest & forest \\
\hline 1754 & 1.29 & 0.81 & 0.00 & & & & \\
\hline 1755 & 1.29 & 0.81 & 0.00 & 1797 & 1.52 & 0.89 & 0.00 \\
\hline 1756 & 1.30 & 0.81 & 0.00 & 1798 & 1.52 & 0.90 & 0.00 \\
\hline 1757 & 1.30 & 0.81 & 0.00 & 1799 & 1.53 & 0.90 & 0.00 \\
\hline 1758 & 1.31 & 0.82 & 0.00 & 1800 & 1.53 & 0.90 & 0.00 \\
\hline 1759 & 1.31 & 0.82 & 0.00 & 1801 & 1.54 & 0.90 & 0.00 \\
\hline 1760 & 1.32 & 0.82 & 0.00 & 1802 & 1.54 & 0.90 & 0.00 \\
\hline 1761 & 1.32 & 0.82 & 0.00 & 1803 & 1.55 & 0.91 & 0.00 \\
\hline 1762 & 1.33 & 0.82 & 0.00 & 1804 & 1.55 & 0.91 & 0.00 \\
\hline 1763 & 1.33 & 0.83 & 0.00 & 1805 & 1.56 & 0.91 & 0.00 \\
\hline 1764 & 1.34 & 0.83 & 0.00 & 1806 & 1.56 & 0.91 & 0.00 \\
\hline 1765 & 1.34 & 0.83 & 0.00 & 1807 & 1.57 & 0.91 & 0.00 \\
\hline 1766 & 1.35 & 0.83 & 0.00 & 1808 & 1.58 & 0.92 & 0.00 \\
\hline 1767 & 1.35 & 0.83 & 0.00 & 1809 & 1.58 & 0.92 & 0.00 \\
\hline 1768 & 1.36 & 0.84 & 0.00 & 1810 & 1.59 & 0.92 & 0.00 \\
\hline 1769 & 1.37 & 0.84 & 0.00 & 1811 & 1.59 & 0.92 & 0.00 \\
\hline 1770 & 1.37 & 0.84 & 0.00 & 1812 & 1.60 & 0.92 & 0.00 \\
\hline 1771 & 1.38 & 0.84 & 0.00 & 1813 & 1.60 & 0.93 & 0.00 \\
\hline 1772 & 1.38 & 0.84 & 0.00 & 1814 & 1.61 & 0.93 & 0.00 \\
\hline 1773 & 1.39 & 0.85 & 0.00 & 1815 & 1.61 & 0.93 & 0.00 \\
\hline 1774 & 1.39 & 0.85 & 0.00 & 1816 & 1.62 & 0.93 & 0.00 \\
\hline 1775 & 1.40 & 0.85 & 0.00 & 1817 & 1.62 & 0.93 & 0.00 \\
\hline 1776 & 1.40 & 0.85 & 0.00 & 1818 & 1.63 & 0.94 & 0.00 \\
\hline 1777 & 1.41 & 0.85 & 0.00 & 1819 & 1.63 & 0.94 & 0.00 \\
\hline 1778 & 1.41 & 0.86 & 0.00 & 1820 & 1.64 & 0.94 & 0.00 \\
\hline 1779 & 1.42 & 0.86 & 0.00 & 1821 & 1.64 & 0.94 & 0.00 \\
\hline 1780 & 1.42 & 0.86 & 0.00 & 1822 & 1.65 & 0.94 & 0.00 \\
\hline 1781 & 1.43 & 0.86 & 0.00 & 1823 & 1.66 & 0.95 & 0.00 \\
\hline 1782 & 1.44 & 0.86 & 0.00 & 1824 & 1.66 & 0.95 & 0.00 \\
\hline 1783 & 1.44 & 0.87 & 0.00 & 1825 & 1.67 & 0.95 & 0.00 \\
\hline 1784 & 1.45 & 0.87 & 0.00 & 1826 & 1.67 & 0.95 & 0.00 \\
\hline 1785 & 1.45 & 0.87 & 0.00 & 1827 & 1.68 & 0.95 & 0.00 \\
\hline 1786 & 1.46 & 0.87 & 0.00 & 1828 & 1.68 & 0.96 & 0.00 \\
\hline 1787 & 1.46 & 0.87 & 0.00 & 1829 & 1.69 & 0.96 & 0.00 \\
\hline 1788 & 1.47 & 0.88 & 0.00 & 1830 & 1.69 & 0.96 & 0.00 \\
\hline 1789 & 1.47 & 0.88 & 0.00 & 1831 & 1.70 & 0.96 & 0.00 \\
\hline 1790 & 1.48 & 0.88 & 0.00 & 1832 & 1.70 & 0.96 & 0.00 \\
\hline 1791 & 1.48 & 0.88 & 0.00 & 1833 & 1.71 & 0.97 & 0.00 \\
\hline
\end{tabular}




$\begin{array}{llll}1834 & 1.71 & 0.97 & 0.00 \\ 1835 & 1.72 & 0.97 & 0.00 \\ 1836 & 1.73 & 0.97 & 0.00 \\ 1837 & 1.73 & 0.97 & 0.00 \\ 1838 & 1.74 & 0.98 & 0.00 \\ 1839 & 1.74 & 0.98 & 0.00 \\ 1840 & 1.75 & 0.98 & 0.00 \\ 1841 & 1.75 & 0.98 & 0.00 \\ 1842 & 1.76 & 0.98 & 0.00 \\ 1843 & 1.76 & 0.99 & 0.00\end{array}$

South and Southeast Asia — Timber Harvest (continued)

\begin{tabular}{|c|c|c|c|c|c|c|c|}
\hline & copical & Tropical & $\mathrm{T}$ & 1875 & 2.00 & 1.20 & 0.00 \\
\hline & moist & seasonal & open & 1876 & 2.02 & 1.21 & 0.00 \\
\hline Year & forest & forest & forest & 1877 & 2.04 & 1.22 & 0.00 \\
\hline & & & & 1878 & 2.06 & 1.24 & 0.00 \\
\hline 1844 & 1.77 & 0.99 & 0.00 & 1879 & 2.08 & 1.25 & 0.00 \\
\hline 1845 & 1.77 & 0.99 & 0.00 & 1880 & 2.10 & 1.26 & 0.00 \\
\hline 1846 & 1.78 & 0.99 & 0.00 & 1881 & 2.12 & 1.27 & 0.00 \\
\hline 1847 & 1.78 & 0.99 & 0.00 & 1882 & 2.14 & 1.28 & 0.00 \\
\hline 1848 & 1.79 & 1.00 & 0.00 & 1883 & 2.16 & 1.30 & 0.00 \\
\hline 1849 & 1.80 & 1.00 & 0.00 & 1884 & 2.18 & 1.31 & 0.00 \\
\hline 1850 & 1.80 & 1.00 & 0.00 & 1885 & 2.20 & 1.32 & 0.00 \\
\hline 1851 & 1.81 & 1.01 & 0.00 & 1886 & 2.22 & 1.33 & 0.00 \\
\hline 1852 & 1.82 & 1.02 & 0.00 & 1887 & 2.24 & 1.34 & 0.00 \\
\hline 1853 & 1.82 & 1.02 & 0.00 & 1888 & 2.26 & 1.36 & 0.00 \\
\hline 1854 & 1.83 & 1.03 & 0.00 & 1889 & 2.28 & 1.37 & 0.00 \\
\hline 1855 & 1.84 & 1.04 & 0.00 & 1890 & 2.30 & 1.38 & 0.00 \\
\hline 1856 & 1.85 & 1.05 & 0.00 & & Tropical & Tropical & Tropical \\
\hline 1857 & 1.86 & 1.06 & 0.00 & & moist & seasonal & open \\
\hline 1858 & 1.86 & 1.06 & 0.00 & Year & forest & forest & forest \\
\hline 1859 & 1.87 & 1.07 & 0.00 & & & & \\
\hline 1860 & 1.88 & 1.08 & 0.00 & 1891 & 2.32 & 1.39 & 0.00 \\
\hline 1861 & 1.89 & 1.09 & 0.00 & 1892 & 2.34 & 1.40 & 0.00 \\
\hline 1862 & 1.90 & 1.10 & 0.00 & 1893 & 2.36 & 1.42 & 0.00 \\
\hline 1863 & 1.90 & 1.10 & 0.00 & 1894 & 2.38 & 1.43 & 0.00 \\
\hline 1864 & 1.91 & 1.11 & 0.00 & 1895 & 2.40 & 1.44 & 0.00 \\
\hline 1865 & 1.92 & 1.12 & 0.00 & 1896 & 2.42 & 1.45 & 0.00 \\
\hline 1866 & 1.93 & 1.13 & 0.00 & 1897 & 2.44 & 1.46 & 0.00 \\
\hline 1867 & 1.94 & 1.14 & 0.00 & 1898 & 2.46 & 1.48 & 0.00 \\
\hline 1868 & 1.94 & 1.14 & 0.00 & 1899 & 2.48 & 1.49 & 0.00 \\
\hline 1869 & 1.95 & 1.15 & 0.00 & 1900 & 2.50 & 1.50 & 0.00 \\
\hline 1870 & 1.96 & 1.16 & 0.00 & 1901 & 2.52 & 1.51 & 0.00 \\
\hline 1871 & 1.97 & 1.17 & 0.00 & 1902 & 2.54 & 1.52 & 0.00 \\
\hline 1872 & 1.98 & 1.18 & 0.00 & 1903 & 2.56 & 1.52 & 0.00 \\
\hline 1873 & 1.98 & 1.18 & 0.00 & 1904 & 2.58 & 1.53 & 0.00 \\
\hline 1874 & 1.99 & 1.19 & 0.00 & 1905 & 2.60 & 1.54 & 0.00 \\
\hline
\end{tabular}




$\begin{array}{llll}1906 & 2.62 & 1.55 & 0.00 \\ 1907 & 2.64 & 1.56 & 0.00 \\ 1908 & 2.66 & 1.56 & 0.00 \\ 1909 & 2.68 & 1.57 & 0.00 \\ 1910 & 2.70 & 1.58 & 0.00 \\ 1911 & 2.72 & 1.59 & 0.00 \\ 1912 & 2.74 & 1.60 & 0.00 \\ 1913 & 2.76 & 1.60 & 0.00 \\ 1914 & 2.78 & 1.61 & 0.00 \\ 1915 & 2.80 & 1.62 & 0.00 \\ 1916 & 2.82 & 1.63 & 0.00 \\ 1917 & 2.84 & 1.64 & 0.00 \\ 1918 & 2.86 & 1.64 & 0.00 \\ 1919 & 2.88 & 1.65 & 0.00 \\ 1920 & 2.90 & 1.66 & 0.00 \\ 1921 & 2.92 & 1.67 & 0.00 \\ 1922 & 2.94 & 1.68 & 0.00 \\ 1923 & 2.96 & 1.68 & 0.00 \\ 1924 & 2.98 & 1.69 & 0.00 \\ 1925 & 3.00 & 1.70 & 0.00 \\ 1926 & 3.04 & 1.71 & 0.00 \\ 1927 & 3.08 & 1.72 & 0.00 \\ 1928 & 3.12 & 1.74 & 0.00 \\ 1929 & 3.16 & 1.75 & 0.00 \\ 1930 & 3.20 & 1.76 & 0.00 \\ 1931 & 3.24 & 1.77 & 0.00 \\ 1932 & 3.28 & 1.78 & 0.00 \\ 1933 & 3.32 & 1.80 & 0.00 \\ 1934 & 3.36 & 1.81 & 0.00 \\ 1935 & 3.40 & 1.82 & 0.00 \\ 1936 & 3.44 & 1.83 & 0.00 \\ 1937 & 3.48 & 1.84 & 0.00\end{array}$

South and Southeast Asia — Timber Harvest (continued)

\begin{tabular}{|c|c|c|c|c|c|c|c|}
\hline & copical & Tropical & $\operatorname{Tr}$ & 1947 & 3.88 & 1.96 & 0.00 \\
\hline & moist & seasonal & open & 1948 & 3.92 & 1.98 & 0.00 \\
\hline Year & forest & forest & forest & 1949 & 3.96 & 1.99 & 0.00 \\
\hline & & & & 1950 & 4.00 & 2.00 & 0.00 \\
\hline 1938 & 3.52 & 1.86 & 0.00 & 1951 & 4.50 & 2.25 & 0.00 \\
\hline 1939 & 3.56 & 1.87 & 0.00 & 1952 & 5.00 & 2.50 & 0.00 \\
\hline 1940 & 3.60 & 1.88 & 0.00 & 1953 & 5.50 & 2.75 & 0.00 \\
\hline 1941 & 3.64 & 1.89 & 0.00 & 1954 & 6.00 & 3.00 & 0.00 \\
\hline 1942 & 3.68 & 1.90 & 0.00 & 1955 & 6.50 & 3.25 & 0.00 \\
\hline 1943 & 3.72 & 1.92 & 0.00 & 1956 & 7.00 & 3.50 & 0.00 \\
\hline 1944 & 3.76 & 1.93 & 0.00 & 1957 & 7.50 & 3.75 & 0.00 \\
\hline 1945 & 3.80 & 1.94 & 0.00 & 1958 & 8.00 & 4.00 & 0.00 \\
\hline 46 & 3.84 & 1.95 & 0.00 & 1959 & 8.50 & 4.25 & 0.00 \\
\hline
\end{tabular}




\begin{tabular}{|c|c|c|c|}
\hline 1960 & 9.00 & 4.50 & 0.00 \\
\hline 1961 & 9.70 & 4.65 & 0.00 \\
\hline 1962 & 10.40 & 4.80 & 0.00 \\
\hline 1963 & 11.10 & 4.95 & 0.00 \\
\hline 1964 & 11.80 & 5.10 & 0.00 \\
\hline 1965 & 12.50 & 5.25 & 0.00 \\
\hline 1966 & 13.20 & 5.40 & 0.00 \\
\hline 1967 & 13.90 & 5.55 & 0.00 \\
\hline 1968 & 14.60 & 5.70 & 0.00 \\
\hline 1969 & 15.30 & 5.85 & 0.00 \\
\hline 1970 & 16.00 & 6.00 & 0.00 \\
\hline 1971 & 16.50 & 6.30 & 0.00 \\
\hline 1972 & 17.00 & 6.60 & 0.00 \\
\hline 1973 & 17.50 & 6.90 & 0.00 \\
\hline 1974 & 18.00 & 7.20 & 0.00 \\
\hline 1975 & 18.50 & 7.50 & 0.00 \\
\hline 1976 & 19.00 & 7.80 & 0.00 \\
\hline 1977 & 19.50 & 8.10 & 0.00 \\
\hline 1978 & 20.00 & 8.40 & 0.00 \\
\hline 1979 & 20.50 & 8.70 & 0.00 \\
\hline 1980 & 21.00 & 9.00 & 0.00 \\
\hline 1981 & 21.83 & 9.37 & 0.00 \\
\hline 1982 & 22.67 & 9.73 & 0.00 \\
\hline 1983 & 23.50 & 10.10 & 0.00 \\
\hline 1984 & 24.33 & 10.47 & 0.00 \\
\hline \multicolumn{2}{|c|}{$\begin{array}{r}\text { Tropical } \\
\text { moist }\end{array}$} & $\begin{array}{l}\text { Tropical } \\
\text { seasonal }\end{array}$ & $\begin{array}{r}\text { Tropical } \\
\text { open }\end{array}$ \\
\hline Year & forest & forest & forest \\
\hline 1985 & 25.17 & 10.83 & 0.00 \\
\hline 1986 & 26.00 & 11.20 & 0.00 \\
\hline 1987 & 26.83 & 11.57 & 0.00 \\
\hline 1988 & 27.67 & 11.93 & 0.00 \\
\hline 1989 & 28.50 & 12.30 & 0.00 \\
\hline 1990 & 28.50 & 12.30 & 0.00 \\
\hline 1991 & 31.22 & 13.47 & 0.00 \\
\hline 1992 & 32.00 & 13.81 & 0.00 \\
\hline 1993 & 30.02 & 12.95 & 0.00 \\
\hline 1994 & 28.68 & 12.38 & 0.00 \\
\hline 1995 & 28.49 & 12.29 & 0.00 \\
\hline
\end{tabular}

South and Southeast Asia — Forest Clearing for Croplands (stepped changes between dates) $\left(10^{6}\right.$ hectares per year):

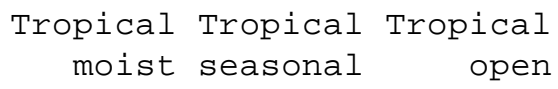




$\begin{array}{llll}\text { Years } & \text { forest } & \text { forest } & \text { forest } \\ & & & \\ 1750-1799 & 0.000 & 0.000 & 0.000 \\ 1800-1809 & 0.008 & 0.272 & 0.000 \\ 1810-1829 & 0.027 & 0.268 & 0.000 \\ 1830-1849 & 0.038 & 0.459 & 0.000 \\ 1850-1869 & 0.040 & 0.450 & 0.000 \\ 1870-1889 & 0.081 & 0.626 & 0.000 \\ 1890-1904 & 0.136 & 0.702 & 0.040 \\ 1905-1911 & 0.178 & 0.708 & 0.070 \\ 1912-1914 & 0.178 & 0.468 & 0.070 \\ 1915-1924 & 0.238 & 0.446 & 0.060 \\ 1925-1934 & 0.230 & 0.409 & 0.058 \\ 1935-1944 & 0.227 & 0.630 & 0.007 \\ 1945-1947 & 0.347 & 1.194 & 0.157 \\ 1948-1951 & 0.347 & 1.184 & 0.157 \\ 1952-1956 & 0.357 & 0.576 & 0.123 \\ 1957-1961 & 0.160 & 0.804 & 0.240 \\ 1962-1966 & 0.293 & 0.676 & 0.187 \\ 1967-1969 & 0.360 & 0.597 & 0.263 \\ 1970-1971 & 0.360 & 0.417 & 0.263 \\ 1972-1974 & 0.576 & 0.780 & 0.184 \\ 1975-1979 & 1.200 & 0.860 & 0.103 \\ 1980-1985 & 2.711 & 1.035 & 0.207 \\ 1986-1990 & 2.721 & 0.765 & 0.206 \\ 1991-1995 & 2.271 & 0.639 & 0.172\end{array}$


South and Southeast Asia — Lands in Shifting Cultivation Cycle (linear change between dates) $\left(10^{6}\right.$ hectares per year):

$\begin{array}{lrrr}\text { Years } & \begin{array}{r}\text { Tropical } \\ \text { moist } \\ \text { forest }\end{array} & \begin{array}{r}\text { Tropical } \\ \text { seasonal } \\ \text { forest }\end{array} & \begin{array}{r}\text { Tropical } \\ \text { open } \\ \text { forest }\end{array} \\ 1750-1801 & 3.73 & 0.00 & 0.35 \\ 1802-1939 & 3.73 & 0.00 & 0.35 \\ 1940-1944 & 3.85 & 0.00 & 0.36 \\ 1945-1949 & 3.93 & 0.00 & 0.36 \\ 1950-1954 & 4.09 & 0.00 & 0.37 \\ 1955-1959 & 4.17 & 0.00 & 0.38 \\ 1960-1964 & 4.35 & 0.00 & 0.39 \\ 1965-1969 & 4.63 & 0.00 & 0.40 \\ 1970-1974 & 4.85 & 0.00 & 0.40 \\ 1975-1979 & 5.25 & 0.00 & 0.42 \\ 1980-1985 & 5.69 & 0.00 & 0.42 \\ 1986-1990 & 6.15 & 0.00 & 0.44 \\ 1991-1995 & 6.25 & 0.00 & 0.44\end{array}$

\section{China}

The following values replace the data in files chin-rat.* in Houghton and Hackler (1995).

China — Clearing for Croplands $\left(10^{6}\right.$ hectares per year):

$\begin{array}{lrrrr}\begin{array}{r}\text { Temperate } \\ \text { evergreen } \\ \text { forest }\end{array} & \begin{array}{r}\text { Temperate } \\ \text { deciduous } \\ \text { forest }\end{array} & \begin{array}{r}\text { Tropical } \\ \text { moist } \\ \text { forest }\end{array} & \begin{array}{r}\text { Temperate } \\ \text { grassland }\end{array} \\ 1700-1701 & 0.000 & 0.000 & 0.000 & 0.000 \\ 1702-1872 & 0.046 & 0.182 & 0.030 & 0.046 \\ 1873-1912 & 0.038 & 0.152 & 0.025 & 0.038 \\ 1913-1932 & 0.055 & 0.220 & 0.037 & 0.055 \\ 1933-1953 & 0.000 & 0.300 & 0.049 & 0.150 \\ 1954-1964 & 0.000 & 0.470 & 0.112 & 0.540 \\ 1965-1969 & 0.000 & 0.480 & 0.097 & 0.390 \\ 1970-1979 & 0.000 & 0.000 & 0.065 & 0.590 \\ 1980-1990 & 0.000 & 0.000 & 0.000 & 0.000\end{array}$


China - Afforestation $\left(10^{6}\right.$ hectares per year):

$\begin{array}{ll}\text { Years } & \text { Temperate evergreen forest } \\ 1700-1710 & 0.00 \\ 1951-1970 & 1.40 \\ 1971-1980 & 2.40 \\ 1981-1990 & 0.38\end{array}$

China - Wood Harvest ( $10^{6} \mathrm{Mg} \mathrm{C}$ per year):

$\begin{array}{lrrr}\begin{array}{r}\text { Tropical } \\ \text { moist } \\ \text { forest }\end{array} & \begin{array}{r}\text { Temperate } \\ \text { evergreen } \\ \text { forest }\end{array} & \begin{array}{r}\text { Temperate } \\ \text { deciduous } \\ \text { forest }\end{array} \\ 1700-1859 & 0.8 & 3.7 & 4.6 \\ 1860-1874 & 2.0 & 10.0 & 12.0 \\ 1875-1899 & 2.0 & 10.0 & 12.0 \\ 1900-1924 & 2.0 & 11.0 & 13.0 \\ 1925-1949 & 3.0 & 12.0 & 15.0 \\ 1950-1969 & 3.0 & 13.0 & 16.0 \\ 1970-1979 & 5.0 & 22.0 & 27.0 \\ 1980-1980 & 5.0 & 25.0 & 31.0 \\ 1981-1990 & 6.7 & 31.7 & 38.6\end{array}$

China — Lands in Pasture ( $10^{6}$ hectares per year):

$\begin{array}{lc}\text { Years } & \text { Temperate grassland } \\ 1700-1701 & 1.0 \\ 1702-1780 & 0.9 \\ 1781-1860 & 0.6 \\ 1861-1875 & 0.6 \\ 1876-1900 & 0.8 \\ 1901-1925 & 0.8 \\ 1926-1950 & 2.1 \\ 1951-1970 & 0.8 \\ 1971-1980 & 0.4 \\ 1981-1990 & 0.0\end{array}$


Houghton \& Hackler 2001, CDIAC NDP-050/R1 (http://cdiac.esd.ornl.gov/ndps/ndp050.html)

\section{Europe}

The following values replace the data in files euro-rat.* in Houghton and Hackler (1995).

Europe - Clearing for Croplands $\left(10^{6}\right.$ hectares per year):

$\begin{array}{lrrrr}\text { Years } & \begin{array}{r}\text { Temperate } \\ \text { evergreen } \\ \text { forest }\end{array} & \begin{array}{r}\text { Temperate } \\ \text { deciduous } \\ \text { forest }\end{array} & \begin{array}{r}\text { Boreal } \\ \text { forest }\end{array} & \begin{array}{r}\text { Temperate } \\ \text { grassland }\end{array} \\ 1700-1701 & 0.0600 & 0.0600 & 0.0500 & 0.2600 \\ 1702-1869 & 0.0600 & 0.0600 & 0.0500 & 0.2600 \\ 1870-1959 & 0.0200 & 0.0200 & 0.0170 & 0.0860 \\ 1960-1961 & 0.0000 & 0.0000 & 0.0000 & 0.0000 \\ 1962-1969 & 0.0000 & 0.0000 & 0.0000 & 0.0000 \\ 1970-1974 & -0.2000 & -0.2000 & -0.2000 & -0.2000 \\ 1975-1980 & -0.2000 & -0.2000 & -0.2000 & -0.2000 \\ 1981-1990 & -0.0506 & -0.0506 & -0.0506 & -0.0506\end{array}$

Europe - Afforestation $\left(10^{6}\right.$ hectares per year):

$\begin{array}{lc}\text { Years } & \text { Temperate evergreen forest } \\ 1700-1710 & 0.00 \\ 1711-1711 & 0.00 \\ 1712-1800 & 0.00 \\ 1801-1849 & 0.02 \\ 1850-1875 & 0.02 \\ 1876-1900 & 0.03 \\ 1901-1925 & 0.05 \\ 1926-1950 & 0.08 \\ 1951-1980 & 0.08 \\ 1981-1990 & 0.08\end{array}$

Europe - Wood Harvest $\left(10^{6} \mathrm{Mg} \mathrm{C}\right.$ per year):

$\begin{array}{lrrr}\text { Temperate } & \begin{array}{r}\text { Temperate } \\ \text { evergreen } \\ \text { feciduous } \\ \text { forest }\end{array} & \begin{array}{r}\text { Boreal } \\ \text { forest }\end{array} \\ 1700 & 12.0 & 10.0 & 2.0 \\ 1860 & 26.0 & 22.0 & 5.0 \\ 1875 & 29.0 & 24.0 & 5.0 \\ 1900 & 35.0 & 30.0 & 6.0 \\ 1925 & 44.0 & 37.0 & 8.0\end{array}$




$\begin{array}{llll}1950 & 41.0 & 35.0 & 7.0 \\ 1970 & 49.0 & 41.0 & 8.0 \\ 1980 & 44.0 & 38.0 & 8.0 \\ 1981-1990 & 51.8 & 43.9 & 9.1\end{array}$

Europe — Lands in Pasture $\left(10^{6}\right.$ hectares per year):

$\begin{array}{lc}\text { Years } & \text { Temperate grassland } \\ 1700-1701 & 0.05 \\ 1702-1780 & 0.05 \\ 1781-1860 & 0.05 \\ 1861-1875 & 0.05 \\ 1876-1900 & 0.05 \\ 1901-1925 & 0.05 \\ 1926-1950 & 0.00 \\ 1951-1970 & 0.00 \\ 1971-1980 & 0.00 \\ 1981-1990 & 0.00\end{array}$

\section{Former Soviet Union}

The following values replace the data in files fsu-rat.* in Houghton and Hackler (1995).

Former Soviet Union — Clearing for Croplands $\left(10^{6}\right.$ hectares per year):

$\begin{array}{lcc}\text { Years } & \text { Temperate deciduous forest } & \text { Temperate grassland } \\ 1700-1860 & 0.288 & 0.206 \\ 1861-1911 & 0.098 & 0.588 \\ 1912-1939 & 0.148 & 1.111 \\ 1940-1949 & -0.333 & -0.111 \\ 1950-1957 & 1.429 & 5.714 \\ 1958-1968 & 0.364 & 0.636 \\ 1969-1970 & -0.065 & -0.065 \\ 1971-1974 & -0.065 & -0.065 \\ 1975-1976 & 0.038 & 0.038 \\ 1977-1980 & 0.038 & 0.038 \\ 1981-1984 & 0.022 & 0.022 \\ 1985-1990 & -0.072 & -0.409\end{array}$


Former Soviet Union — Timber Harvest $\left(10^{6} \mathrm{Mg} \mathrm{C}\right.$ per year):

$\begin{array}{lcc}\text { Years } & \text { Temperate deciduous forest } & \text { Boreal forest } \\ 1700-1701 & 0.00 & 0.00 \\ 1702-1850 & 0.36 & 1.44 \\ 1851-1919 & 3.72 & 14.88 \\ 1914-1932 & 9.88 & 39.52 \\ 1933-1945 & 10.10 & 40.40 \\ 1946-1960 & 22.18 & 88.72 \\ 1961-1970 & 23.10 & 92.40 \\ 1971-1975 & 22.26 & 89.04 \\ 1976-1980 & 21.42 & 85.68 \\ 1981-1985 & 21.42 & 85.68 \\ 1986-1990 & 23.96 & 95.84\end{array}$

Former Soviet Union — Fuelwood Harvest $\left(10^{6} \mathrm{Mg} \mathrm{C}\right.$ per year):

$\begin{array}{lcc}\text { Years } & \text { Temperate deciduous forest } & \text { Boreal forest } \\ 1700-1701 & 0.000 & 0.000 \\ 1702-1850 & 14.364 & 23.436 \\ 1851-1919 & 26.448 & 43.152 \\ 1914-1932 & 25.422 & 41.478 \\ 1933-1945 & 15.960 & 26.040 \\ 1946-1960 & 4.560 & 7.440 \\ 1961-1970 & 4.218 & 6.882 \\ 1971-1975 & 3.990 & 6.510 \\ 1976-1980 & 3.762 & 6.138 \\ 1981-1990 & 3.762 & 6.138\end{array}$

\section{North Africa and the Middle East}

The following values replace the data in files nafm-rat.* in Houghton and Hackler (1995).

North Africa and the Middle East - Clearing for Croplands $\left(10^{6}\right.$ hectares per year):

$\begin{array}{lrrrr}\begin{array}{r}\text { Temperate } \\ \text { evergreen } \\ \text { Years }\end{array} & \begin{array}{r}\text { Tropical } \\ \text { grassland }\end{array} & \begin{array}{r}\text { Tropical } \\ \text { Desert } \\ \text { sorub } \\ \text { woodland/ } \\ \text { shrubland }\end{array} \\ 1700-1701 & 0.000 & 0.000 & 0.000 & 0.000 \\ 1702-1859 & 0.014 & 0.024 & 0.000 & 0.010 \\ 1860-1899 & 0.066 & 0.111 & 0.000 & 0.044\end{array}$




$\begin{array}{lllll}1900-1924 & 0.091 & 0.151 & 0.000 & 0.060 \\ 1925-1949 & 0.150 & 0.410 & 0.100 & 0.170 \\ 1950-1964 & 0.000 & 0.640 & 0.510 & 0.130 \\ 1965-1979 & 0.000 & 0.730 & 0.590 & 0.150 \\ 1980-1990 & 0.000 & 0.297 & 0.238 & 0.061\end{array}$

North Africa and the Middle East — Wood Harvest (10 $\mathrm{Mg} \mathrm{C}$ per year):

$\begin{array}{lcc}\text { Year } & \text { Temperate evergreen forest } & \text { Tropical moist forest } \\ 1700 & 0.90 & 0.50 \\ 1860 & 1.00 & 1.00 \\ 1875 & 1.00 & 1.00 \\ 1900 & 2.00 & 1.00 \\ 1925 & 2.00 & 1.00 \\ 1950 & 3.00 & 2.00 \\ 1970 & 6.00 & 3.00 \\ 1980 & 7.00 & 4.00 \\ 1990 & 10.94 & 6.25\end{array}$

North Africa and the Middle East — Lands in Pasture ( $10^{6}$ hectares per year):

Years Desert scrub

$\begin{array}{ll}1700-1701 & 0.1 \\ 1702-1780 & 0.1 \\ 1781-1860 & 0.2 \\ 1861-1875 & 0.5 \\ 1876-1900 & 0.7 \\ 1901-1925 & 1.5 \\ 1926-1950 & 2.1 \\ 1951-1970 & 1.3 \\ 1971-1990 & 0.5\end{array}$

\section{North America}

The following values replace the data in files nam-rat.* in Houghton and Hackler (1995).

North America - Clearing for Croplands $\left(10^{6}\right.$ hectares per year):

\begin{tabular}{|c|c|c|c|c|c|c|c|}
\hline \multirow[b]{2}{*}{ Years } & \multicolumn{2}{|c|}{ Temperate forest } & \multicolumn{2}{|c|}{$\begin{array}{c}\text { Temperate forest } \\
\text { (abandonment) }\end{array}$} & \multirow{2}{*}{$\begin{array}{l}\text { Boreal } \\
\text { forest }\end{array}$} & \multirow{2}{*}{$\begin{array}{l}\text { Temperate } \\
\text { woodland/ } \\
\text { shrubland }\end{array}$} & \multirow{2}{*}{$\begin{array}{l}\text { Temperate } \\
\text { grassland }\end{array}$} \\
\hline & evergreen & deciduous & evergreen & deciduous & & & \\
\hline $1700-1701$ & 0.135 & 0.315 & -0.045 & -0.105 & 0.00 & 0.000 & 0.000 \\
\hline $1702-1849$ & 0.135 & 0.315 & -0.045 & -0.105 & 0.00 & 0.000 & 0.000 \\
\hline
\end{tabular}


Houghton \& Hackler 2001, CDIAC NDP-050/R1 (http://cdiac.esd.ornl.gov/ndps/ndp050.html)

\begin{tabular}{|c|c|c|c|c|c|c|c|}
\hline $1850-1889$ & 0.135 & 0.135 & -0.045 & -0.045 & 0.03 & 0.090 & 1.200 \\
\hline $1890-1927$ & 0.210 & 0.210 & -0.070 & -0.070 & 0.05 & 0.140 & 1.810 \\
\hline $1928-1949$ & 0.045 & 0.045 & -0.015 & -0.015 & 0.01 & 0.030 & 0.400 \\
\hline $1950-1969$ & 0.010 & 0.010 & -0.010 & -0.010 & 0.00 & 0.000 & 0.000 \\
\hline $1970-1979$ & 0.010 & 0.010 & -0.100 & -0.100 & 0.00 & 0.000 & -0.020 \\
\hline $1980-1990$ & -0.122 & -0.122 & 0.000 & 0.000 & 0.00 & 0.000 & -0.027 \\
\hline
\end{tabular}




\begin{tabular}{lrrr}
\multicolumn{5}{c}{ North America - Wood Harvest $\left(10^{6} \mathrm{Mg}\right.$ C p p } \\
Temperate \\
Year & $\begin{array}{r}\text { Temperate } \\
\text { deciduous } \\
\text { forest } \\
\text { forest }\end{array}$ & $\begin{array}{r}\text { Boreal } \\
\text { forest }\end{array}$ \\
1700 & 1.4 & 1.2 & 2.3 \\
1860 & 30.0 & 26.0 & 51.0 \\
1875 & 37.0 & 31.0 & 62.0 \\
1900 & 36.0 & 31.0 & 61.0 \\
1925 & 38.0 & 32.0 & 64.0 \\
1950 & 33.0 & 28.0 & 55.0 \\
1970 & 38.0 & 32.0 & 63.0 \\
1980 & 41.0 & 35.0 & 69.0 \\
1990 & 55.1 & 46.8 & 92.5
\end{tabular}

North America — Lands in Pasture $\left(10^{6}\right.$ hectares per year):

$\begin{array}{lc}\text { Years } & \text { Temperate grassland } \\ & \\ 1700-1701 & 0.3 \\ 1702-1780 & 0.6 \\ 1781-1860 & 0.9 \\ 1861-1875 & 1.8 \\ 1876-1900 & 3.6 \\ 1901-1925 & 1.6 \\ 1926-1950 & 0.1 \\ 1951-1970 & 0.0 \\ 1971-1990 & 0.0\end{array}$

\section{Pacific Developed Region}

The following values replace the data in files pcdv-rat.* in Houghton and Hackler (1995).

Pacific Developed Region — Clearing for Croplands ( $10^{6}$ hectares per year):

$\begin{array}{lrrr}\text { Years } & \begin{array}{r}\text { Tropical } \\ \text { moist } \\ \text { forest }\end{array} & \begin{array}{r}\text { Tropical } \\ \text { grassland }\end{array} & \begin{array}{r}\text { Tropical } \\ \text { woodland/ } \\ \text { shrubland }\end{array} \\ 1700-1869 & 0.001 & 0.004 & 0.002 \\ 1870-1949 & 0.038 & 0.152 & 0.064 \\ 1950-1969 & 0.220 & 0.860 & 0.360 \\ 1970-1979 & 0.030 & 0.120 & 0.050\end{array}$


Houghton \& Hackler 2001, CDIAC NDP-050/R1 (http://cdiac.esd.ornl.gov/ndps/ndp050.html)
$1980-1990$
0.051
2.000
0.084

B-18 
Pacific Developed Region — Wood Harvest $\left(10^{6} \mathrm{Mg} \mathrm{C}\right.$ per year):

$\begin{array}{lrrr}\begin{array}{r}\text { Temperate } \\ \text { evergreen } \\ \text { forest }\end{array} & \begin{array}{r}\text { Temperate } \\ \text { deciduous } \\ \text { forest }\end{array} & \begin{array}{r}\text { Tropical } \\ \text { moist } \\ \text { forest }\end{array} \\ 1700-1701 & 3.8 & 4.5 & 1.2 \\ 1702-1859 & 5.0 & 6.0 & 1.0 \\ 1860-1874 & 5.0 & 6.0 & 2.0 \\ 1875-1899 & 7.0 & 8.0 & 2.0 \\ 1900-1924 & 9.0 & 11.0 & 3.0 \\ 1925-1949 & 12.0 & 14.0 & 4.0 \\ 1950-1969 & 12.0 & 13.0 & 3.0 \\ 1970-1979 & 10.0 & 12.0 & 3.0 \\ 1980-1990 & 6.9 & 8.2 & 2.1\end{array}$

Pacific Developed Region — Lands in Pasture ( $10^{6}$ hectares per year):

$\begin{array}{lrr}\text { Years } & \begin{array}{r}\text { Tropical } \\ \text { grassland }\end{array} & \begin{array}{r}\text { Desert } \\ \text { scrub }\end{array} \\ 1700-1701 & 0.0 & 0.0 \\ 1702-1780 & 0.0 & 0.0 \\ 1781-1860 & 0.0 & 0.0 \\ 1861-1875 & 0.0 & 0.0 \\ 1876-1900 & 0.0 & 0.0 \\ 1901-1925 & 8.0 & 2.6 \\ 1926-1950 & 7.8 & 1.7 \\ 1951-1970 & 0.6 & 0.2 \\ 1971-1990 & 0.6 & 0.2\end{array}$

\section{South and Central America}

The following values replace the data in files scam-rat.* in Houghton and Hackler (1995).

South and Central America — Lands in Forest Plantation ( $10^{6}$ hectares per year):

$\begin{array}{lc}\text { Years } & \text { Tropical seasonal forest } \\ 1800-1948 & 0.000 \\ 1949-1954 & 0.040 \\ 1955-1957 & 0.040 \\ 1958-1962 & 0.079 \\ 1963-1967 & 0.127 \\ 1968-1977 & 0.228\end{array}$


Houghton \& Hackler 2001, CDIAC NDP-050/R1 (http://cdiac.esd.ornl.gov/ndps/ndp050.html)

$\begin{array}{ll}1978-1982 & 0.410 \\ 1983-1990 & 0.535\end{array}$

South and Central America - Clearing for Croplands $\left(10^{6}\right.$ hectares per year):

$\begin{array}{lrrrrrrr} & \begin{array}{r}\text { Tropical } \\ \text { equatorial } \\ \text { forest }\end{array} & \begin{array}{r}\text { Tropical } \\ \text { seasonal } \\ \text { forest }\end{array} & \begin{array}{r}\text { Tropical } \\ \text { woodland }\end{array} & \begin{array}{r}\text { Woniferous } \\ \text { forest }\end{array} & \begin{array}{r}\text { Temperate } \\ \text { broadleaf } \\ \text { forest }\end{array} & \text { Grassland } & \begin{array}{r}\text { Desert } \\ \text { scrub }\end{array} \\ 1800-1849 & 0.0037 & 0.0267 & 0.0147 & 0.0037 & 0.0009 & 0.0414 & 0.0009 \\ 1850-1859 & 0.0037 & 0.0267 & 0.0147 & 0.0037 & 0.0009 & 0.0414 & 0.0009 \\ 1860-1869 & 0.0082 & 0.0595 & 0.0328 & 0.0082 & 0.0021 & 0.0923 & 0.0021 \\ 1870-1879 & 0.0082 & 0.0595 & 0.0328 & 0.0082 & 0.0021 & 0.0923 & 0.0021 \\ 1880-1889 & 0.0264 & 0.1910 & 0.1056 & 0.0264 & 0.0066 & 0.2970 & 0.0066 \\ 1890-1899 & 0.0240 & 0.1740 & 0.0960 & 0.0240 & 0.0060 & 0.2700 & 0.0060 \\ 1900-1909 & 0.0864 & 0.6260 & 0.3456 & 0.0864 & 0.0216 & 0.9720 & 0.0216 \\ 1910-1919 & -0.0032 & -0.0232 & -0.0128 & -0.0032 & -0.0008 & -0.0360 & -0.0008 \\ 1920-1929 & 0.0616 & 0.4470 & 0.2464 & 0.0616 & 0.0154 & 0.6930 & 0.0154 \\ 1930-1939 & 0.0572 & 0.4150 & 0.2288 & 0.0572 & 0.0143 & 0.6440 & 0.0143 \\ 1940-1949 & 0.0292 & 0.2120 & 0.1168 & 0.0292 & 0.0073 & 0.3280 & 0.0073 \\ 1950-1959 & 0.0660 & 0.4780 & 0.2640 & 0.0660 & 0.0165 & 0.7420 & 0.0165 \\ 1960-1969 & 0.1740 & 1.2640 & 0.6976 & 0.1744 & 0.0436 & 1.9620 & 0.0436 \\ 1970-1979 & 0.1010 & 0.7310 & 0.4032 & 0.1008 & 0.0252 & 1.1340 & 0.0252 \\ 1980-1982 & 0.0460 & 0.3310 & 1.2480 & 0.0460 & 0.0110 & 0.5130 & 0.0110 \\ 1983-1984 & 0.0460 & 0.3310 & 1.2620 & 0.0460 & 0.0110 & 0.5130 & 0.0110 \\ 1986-1987 & 0.0460 & 0.3310 & 1.2860 & 0.0460 & 0.0110 & 0.5130 & 0.0110 \\ 1988-1989 & 0.0460 & 0.3310 & 0.9940 & 0.0460 & 0.0110 & 0.5130 & 0.0110 \\ 1990 & 0.0460 & 0.3310 & 0.7000 & 0.0460 & 0.0110 & 0.5130 & 0.0110\end{array}$

\section{South and Central America — Lands in Pasture ( $10^{6}$ hectares per year):}

\begin{tabular}{|c|c|c|c|c|c|c|c|}
\hline Years & $\begin{array}{r}\text { Tropical } \\
\text { equatorial } \\
\text { forest }\end{array}$ & $\begin{array}{r}\text { Tropical } \\
\text { seasonal } \\
\text { forest }\end{array}$ & $\begin{array}{l}\text { Tropical } \\
\text { woodland }\end{array}$ & $\begin{array}{r}\text { Warm } \\
\text { coniferous } \\
\text { forest }\end{array}$ & $\begin{array}{r}\text { Temperate } \\
\text { broadleaf } \\
\text { forest }\end{array}$ & Grassland & $\begin{array}{r}\text { Desert } \\
\text { scrub }\end{array}$ \\
\hline $1800-1859$ & 0.000 & 0.000 & 0.735 & 0.000 & 0.000 & 1.323 & 0.042 \\
\hline $1860-1869$ & 0.005 & 0.090 & -0.210 & 0.005 & 0.000 & -0.378 & -0.012 \\
\hline $1870-1879$ & 0.000 & 0.000 & 0.525 & 0.000 & 0.000 & 0.945 & 0.030 \\
\hline $1880-1889$ & 0.005 & 0.090 & 1.330 & 0.005 & 0.000 & 2.394 & 0.076 \\
\hline $1890-1899$ & 0.005 & 0.090 & 0.000 & 0.005 & 0.000 & 0.000 & 0.000 \\
\hline $1900-1909$ & 0.005 & 0.090 & 0.770 & 0.005 & 0.000 & 1.386 & 0.044 \\
\hline $1910-1919$ & 0.015 & 0.270 & 0.420 & 0.015 & 0.000 & 0.756 & 0.024 \\
\hline $1920-1929$ & 0.015 & 0.270 & -0.280 & 0.015 & 0.000 & -0.504 & -0.016 \\
\hline $1930-1939$ & 0.020 & 0.360 & 0.665 & 0.020 & 0.000 & 1.197 & 0.038 \\
\hline $1940-1949$ & 0.025 & 0.450 & 1.820 & 0.025 & 0.000 & 3.276 & 0.104 \\
\hline $1950-1959$ & 0.045 & 0.810 & 1.715 & 0.045 & 0.000 & 3.087 & 0.098 \\
\hline $1960-1969$ & 0.055 & 0.990 & 2.415 & 0.055 & 0.000 & 4.347 & 0.138 \\
\hline
\end{tabular}


Houghton \& Hackler 2001, CDIAC NDP-050/R1 (http://cdiac.esd.ornl.gov/ndps/ndp050.html)

$\begin{array}{llllllll}1970-1979 & 0.075 & 1.350 & 0.210 & 0.075 & 0.000 & 0.378 & 0.012 \\ 1980-1982 & 0.060 & 1.752 & 0.000 & 0.060 & 0.000 & 0.000 & 0.000 \\ 1983-1985 & 0.060 & 2.259 & 0.000 & 0.060 & 0.000 & 0.000 & 0.000 \\ 1986-1987 & 0.060 & 2.660 & 0.000 & 0.060 & 0.000 & 0.000 & 0.000 \\ 1988-1989 & 0.060 & 2.480 & 0.000 & 0.060 & 0.000 & 0.000 & 0.000 \\ 1990 & 0.060 & 2.039 & 0.000 & 0.060 & 0.000 & 0.000 & 0.000\end{array}$


South and Central America — Wood Harvest (10 $\mathrm{Mg} \mathrm{C}$ per year):

$\begin{array}{lcc}\text { Years } & \text { Tropical seasonal forest } & \text { Warm coniferous forest } \\ 1800-1801 & 0.416 & 0.301 \\ 1802-1874 & 0.518 & 0.378 \\ 1875-1899 & 0.832 & 0.602 \\ 1900-1924 & 1.978 & 1.428 \\ 1925-1949 & 3.953 & 2.860 \\ 1950-1974 & 12.080 & 8.720 \\ 1975-1979 & 18.720 & 13.540 \\ 1980-1984 & 21.420 & 15.500 \\ 1985-1990 & 21.420 & 15.500\end{array}$

South and Central America — Lands in Shifting Cultivation $\left(10^{6}\right.$ hectares per year):

$\begin{array}{lcc}\text { Years } & \text { Tropical seasonal forest } & \text { Tropical woodland } \\ 1800-1939 & 5.1176 & 9.3333 \\ 1940-1944 & 5.1376 & 9.3333 \\ 1945-1949 & 5.2976 & 9.3533 \\ 1950-1954 & 5.4176 & 9.4333 \\ 1955-1959 & 5.5376 & 9.4933 \\ 1960-1964 & 5.6376 & 9.5133 \\ 1965-1969 & 5.8176 & 9.5333 \\ 1970-1974 & 6.0776 & 9.5533 \\ 1975-1979 & 6.3376 & 9.5733 \\ 1980-1984 & 6.6556 & 9.5933 \\ 1985-1999 & 6.6556 & 9.5933\end{array}$




\section{Tropical Africa}

The following values replace the data in files taf-rat.* in Houghton and Hackler (1995).

Tropical Africa - Forest Clearing $\left(10^{6}\right.$ hectares per year):

$\begin{array}{lcc}\text { Years } & \text { Closed forest } & \text { Open forest } \\ 1800-1824 & 0.021 & 0.037 \\ 1825-1849 & 0.029 & 0.051 \\ 1850-1859 & 0.036 & 0.064 \\ 1860-1874 & 0.045 & 0.080 \\ 1875-1887 & 0.054 & 0.096 \\ 1885-1899 & 0.065 & 0.115 \\ 1900-1909 & 0.090 & 0.160 \\ 1910-1919 & 0.126 & 0.224 \\ 1920-1929 & 0.162 & 0.288 \\ 1930-1939 & 0.198 & 0.352 \\ 1940-1949 & 0.324 & 0.576 \\ 1950-1959 & 0.504 & 0.896 \\ 1960-1969 & 0.792 & 1.408 \\ 1970-1977 & 1.080 & 1.920 \\ 1978-1979 & 1.324 & 2.354 \\ 1980-1984 & 1.440 & 2.560 \\ 1985-1989 & 1.836 & 3.264 \\ 1990 & 2.160 & 3.840\end{array}$




\section{APPENDIX C. REGIONAL CARBON-CHANGE COEFFICIENTS}

The following listing provides the regional values and coefficients associated with oxidation and recovery of carbon in vegetation, soils, and forest products. The values in this listing replace the values in the indicated ascii files and binary spreadsheet files in Houghton and Hackler (1995), the previous version of this database.

The values for five kinds of response curve are provided herein: clearing response curve (changes in the carbon in vegetation and soils resulting from the clearing of natural ecosystems for croplands), pasture response curve (changes in the carbon in vegetation and soils resulting from the clearing of natural ecosystems for pasture), forest plantation response curve (changes in carbon in vegetation and soils following the clearing of forests for plantations), shifting cultivation response curve (changes in the carbon in vegetation and soils following the clearing of natural ecosystems for shifting cultivation), and logging response curve (changes in the carbon in vegetation following the harvest of forests and recovery; the amount of carbon in soils was assumed to be unchanged).

The following values are provided in this appendix:

Carbon in undisturbed vegetation ( $\mathrm{Mg} \mathrm{C} / \mathrm{ha}$ ): Carbon content of live vegetation before land-use change (or harvest)

Carbon in crops ( $\mathrm{Mg} \mathrm{C} / \mathrm{ha}$ ): Carbon content of vegetation remaining in the ecosystem during the period of land-use change

Carbon in disturbed vegetation (Mg C/ha): Carbon content of live vegetation, after land-use change (or harvest) but before initial recovery

Carbon in recovered vegetation ( $\mathrm{Mg} \mathrm{C} / \mathrm{ha}$ ): Carbon content of live vegetation, after land-use change (or harvest) and initial recovery, but before it has returned to initial level

Carbon in slash (Mg C/ha): Carbon content of dead vegetation left on-site during land-use change (or harvest)

Carbon in undisturbed soil ( $\mathrm{Mg} \mathrm{C} / \mathrm{ha}$ ): Carbon content of soil before land-use change (or harvest)

Carbon in recovered soil ( $\mathrm{Mg} \mathrm{C} / \mathrm{ha}$ ): Carbon content of soil, after initial oxidation and subsequent recovery, but before it has returned to initial level 
Soil carbon after initial rapid change ( $\mathrm{Mg} \mathrm{C} / \mathrm{ha})$ : Carbon content of soil after initial oxidation resulting from land-use change

Minimum soil carbon (Mg C/ha): Carbon content of soil following both initial (fast) oxidation and subsequent (slow) oxidation

Time for vegetation to return from disturbed to recovered state (year): Length of time, in years, required for carbon content of vegetation, after land-use change (or harvest), to partially return to initial level

Time for vegetation to return from recovered to primary state (year): Length of time, in years, required for carbon content of vegetation, after land-use change (or harvest) and partial recovery, to complete the full return to initial level

Time for vegetation to return from disturbed to primary state (year): Length of time, in years, required for carbon content of vegetation, after land-use change (or harvest), to fully return to initial level

Duration of initial rapid change (year): Length of time, in years, required for initial rapid oxidation of soil carbon after land-use change (or harvest)

Time to minimum soil carbon (year): Length of time, in years, following initial rapid oxidation of soil carbon, until lowest level of soil carbon is achieved

Time for soil carbon to return from minimum to recovered state (year): Length of time, in years, for carbon content of soil to partially return to initial level from lowest level

Time for soil carbon to return from recovered to primary state (year): Length of time, in years, for carbon content of soil to complete full return to initial level

Time for soil carbon to return from minimum to primary state (year): Length of time, in years, for carbon content of soil to fully return to initial level from lowest level

Fraction of harvested vegetation assigned to decay pools (1-year, 10-year, 100-year, and 1000-year): Fraction of carbon in live vegetation removed from site that is oxidized with decay constants of $1 \mathrm{yr}^{-1}$ (fuelwood), $0.1 \mathrm{yr}^{-1}$ (pulp and paper products), $0.01 \mathrm{yr}^{-1}$ (sawn wood, plywood, panels, and lumber), and $0.001 \mathrm{yr}^{-1}$ (elemental carbon from burning), respectively.

Fraction of harvested vegetation left to decay on-site: Fraction of carbon in vegetation that is killed and left on-site (i.e., slash) 
Rate constant for on-site decay (per year): Fraction of remaining carbon in dead vegetation left on-site (i.e., slash) that oxidizes in each year following land-use change (or harvest)

In some cases, the specific meaning of a term will change according to the context (type of ecosystem, land use, or land-use change involved). Thus, "recovery" in the case of clearing for agriculture is measured from time of abandonment. In the case of shifting cultivation it is measured from the onset of the fallow period, and in a logged forest it is measured following the initial harvest. In the case of logging, a "recovered" system is a secondary forest that can be harvested again, and "crop" represents wood harvested. In the case of shifting cultivation, the "disturbed" state refers to the beginning of the fallow cycle, and the "recovered" state refers to the end of the fallow cycle; the "time for vegetation to return from disturbed to recovered state" represents the length of the fallow cycle. 
Houghton \& Hackler 2001, CDIAC NDP-050/R1 (http://cdiac.esd.ornl.gov/ndps/ndp050.html)

South and Southeast Asia (the following values replace the data in files asia-re.* and asia-sh.* in Houghton and Hackler 1995)

South and Southeast Asia - Clearing Response Curve:

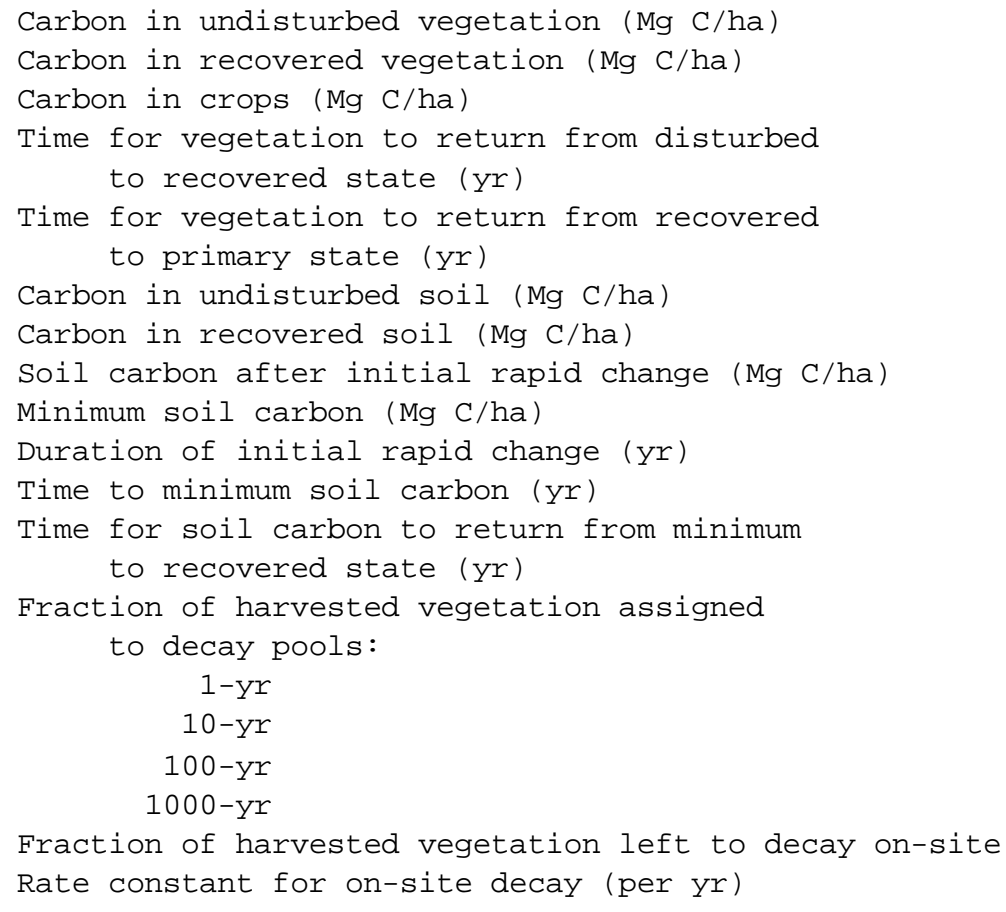

\begin{tabular}{|c|c|c|}
\hline $\begin{array}{r}\text { Tropical } \\
\text { moist } \\
\text { forest }\end{array}$ & $\begin{array}{r}\text { Tropical } \\
\text { seasonal } \\
\text { forest }\end{array}$ & $\begin{array}{r}\text { Tropical } \\
\text { open } \\
\text { forest }\end{array}$ \\
\hline 250 & 150 & 60 \\
\hline 175 & 105 & 42 \\
\hline 5 & 5 & 5 \\
\hline 37 & 29 & 12 \\
\hline 40 & 30 & 40 \\
\hline 120 & 80 & 50 \\
\hline 120 & 80 & 50 \\
\hline 96 & 64 & 40 \\
\hline 84 & 56 & 37 \\
\hline 5 & 5 & 5 \\
\hline 5 & 5 & 5 \\
\hline 40 & 29 & 12 \\
\hline 0.4 & 0.4 & 0.4 \\
\hline 0.1 & 0.1 & 0.1 \\
\hline 0.0 & 0.0 & 0.0 \\
\hline 0.0 & 0.0 & 0.0 \\
\hline 0.5 & 0.5 & 0.5 \\
\hline 0.5 & 0.4 & 0.3 \\
\hline
\end{tabular}




\section{South and Southeast Asia - Shifting Cultivation Response Curve:}

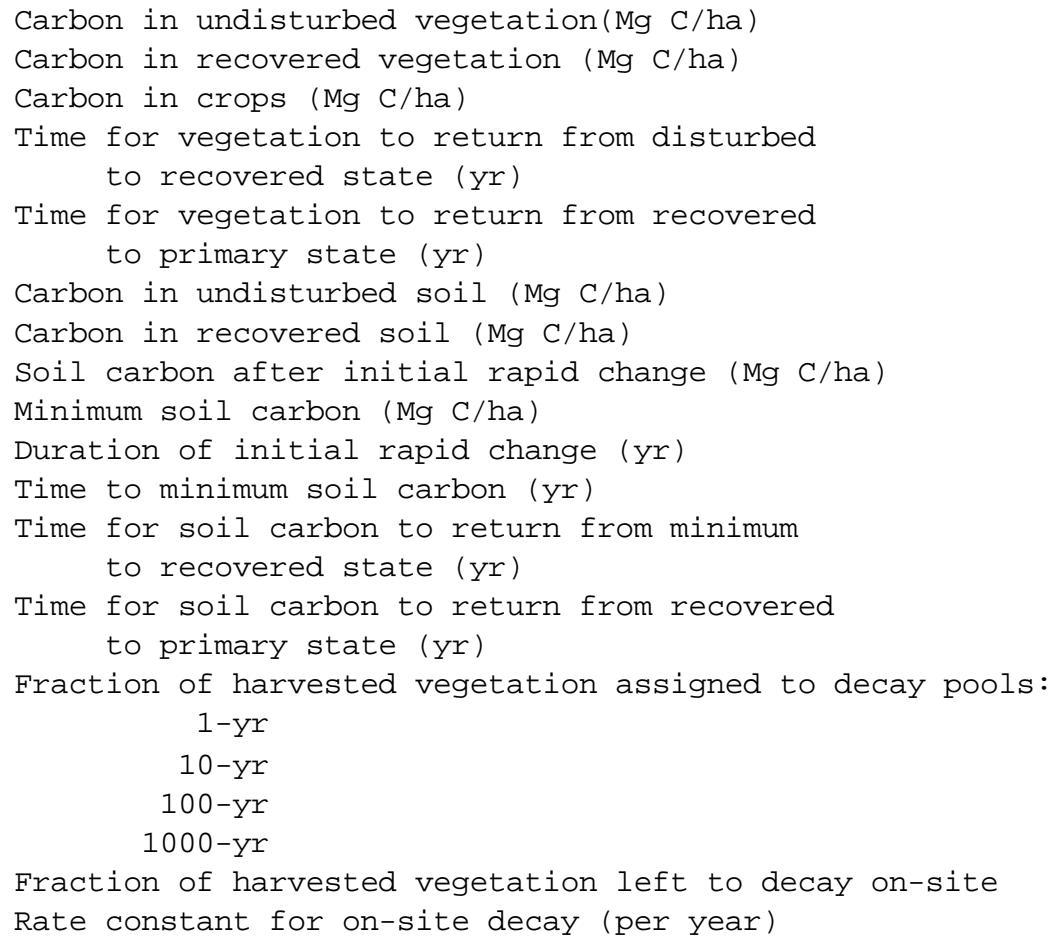

\section{South and Southeast Asia — Logging Response Curve:}

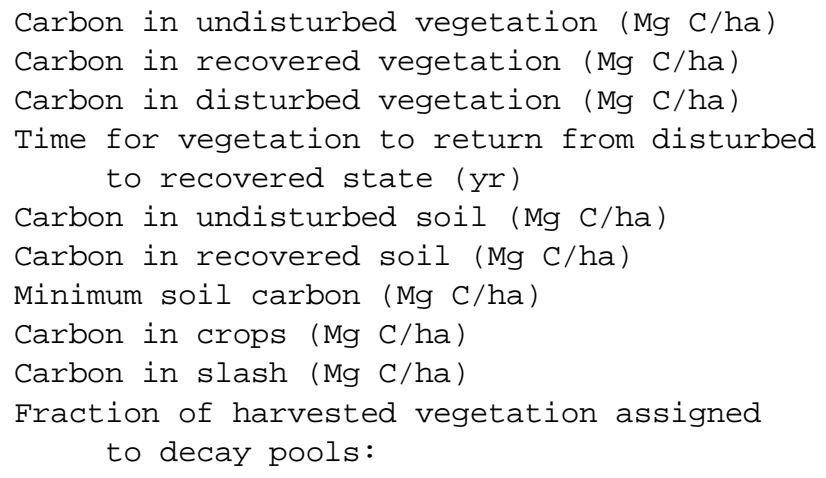

$\begin{array}{cc}\begin{array}{c}\text { Tropical } \\ \text { moist } \\ \text { forest }\end{array} & \begin{array}{c}\text { Tropical } \\ \text { open } \\ \text { forest }\end{array} \\ 250 & 60 \\ 90 & 35 \\ 15 & 5 \\ 15 & 10 \\ & \\ 22 & 2 \\ 120 & 50 \\ 108 & 45 \\ 90 & 38 \\ 90 & 38 \\ 2 & 2 \\ 2 & 2 \\ 15 & 10 \\ & \\ 22 & 2 \\ 0.38 & 0.41 \\ 0.07 & 0.10 \\ 0.00 & 0.00 \\ 0.02 & 0.02 \\ 0.50 & 0.47 \\ & 0.30\end{array}$

$\begin{array}{rr}\begin{array}{r}\text { Tropical } \\ \text { moist } \\ \text { forest }\end{array} & \begin{array}{r}\text { Tropical } \\ \text { seasonal } \\ \text { forest }\end{array} \\ 250 & 150 \\ 175 & 105 \\ 110 & 60 \\ & \\ 20 & 20 \\ 120 & 80 \\ 120 & 80 \\ 120 & 80 \\ 27 & 16 \\ 219 & 131\end{array}$




$\begin{array}{ccc}1-y r & 0.0162 & 0.0162 \\ 10-y r & 0.0088 & 0.0088 \\ 100-y r & 0.1000 & 0.1000 \\ \text { Fraction of harvested vegetation left to decay on-site } & 0.8750 & 0.8750 \\ \text { Rate constant for on-site decay (per year) } & 0.5000 & 0.3000\end{array}$

Former Soviet Union (the following values replace the data in files fsu-re.* and fsulogpt.* in Houghton and Hackler 1995)

Former Soviet Union - Clearing Response Curve:

\begin{tabular}{|c|c|c|c|}
\hline & $\begin{array}{l}\text { Temperate } \\
\text { grassland }\end{array}$ & $\begin{array}{l}\text { Temperate } \\
\text { deciduous } \\
\text { forest }\end{array}$ & $\begin{array}{l}\text { Boreal } \\
\text { forest }\end{array}$ \\
\hline Carbon in undisturbed vegetation ( $\mathrm{Mg} \mathrm{C} / \mathrm{ha}$ ) & 10 & 135 & 90 \\
\hline Carbon in recovered vegetation ( $\mathrm{Mg} \mathrm{C} / \mathrm{ha}$ ) & 10 & 108 & 72 \\
\hline Carbon in crops (Mg $\mathrm{C} / \mathrm{ha}$ ) & 5 & 5 & 5 \\
\hline $\begin{array}{l}\text { Time for vegetation to return from disturbed } \\
\text { to recovered state (yr) }\end{array}$ & 10 & 40 & 80 \\
\hline $\begin{array}{l}\text { Time for vegetation to return from recovered } \\
\text { to primary state (yr) }\end{array}$ & 5 & 21 & 43 \\
\hline Carbon in undisturbed soil (Mg C/ha) & 189 & 134 & 206 \\
\hline Carbon in recovered soil (Mg $\mathrm{C} / \mathrm{ha}$ ) & 189 & 134 & 206 \\
\hline Soil carbon after initial rapid change ( $\mathrm{Mg} \mathrm{C} / \mathrm{ha}$ ) & 161 & 114 & 175 \\
\hline Minimum soil carbon (Mg C/ha) & 151 & 107 & 165 \\
\hline Duration of initial rapid change (yr) & 15 & 10 & 15 \\
\hline Time to minimum soil carbon (yr) & 30 & 30 & 65 \\
\hline $\begin{array}{l}\text { Time for soil carbon to return from minimum } \\
\text { to recovered state (yr) }\end{array}$ & 45 & 40 & 80 \\
\hline $\begin{array}{l}\text { Time for soil carbon to return from recovered } \\
\text { to primary state ( } y r)\end{array}$ & 5 & 21 & 43 \\
\hline $\begin{array}{l}\text { Fraction of harvested vegetation assigned to decay pool } \\
\qquad 1-y r\end{array}$ & 0.4 & 0.48 & 0.48 \\
\hline $10-\mathrm{yr}$ & 0.1 & 0.24 & 0.24 \\
\hline $100-\mathrm{yr}$ & 0.0 & 0.08 & 0.08 \\
\hline $1000-y r$ & 0.0 & 0.00 & 0.00 \\
\hline Fraction of harvested vegetatic & 0.5 & 0.20 & 0.20 \\
\hline Rate constant for on-site decay & 0.5 & 0.04 & 0.05 \\
\hline
\end{tabular}

Former Soviet Union — Logging Response Curve:

\begin{tabular}{|c|c|c|c|c|}
\hline & Fuelwood & arvest & Timber & rvest \\
\hline & Temperate & & perate & \\
\hline & deciduous & Boreal & deciduous & Boreal \\
\hline & forest & forest & forest & forest \\
\hline Carbon in undisturbed vegetation ( $\mathrm{Mg} \mathrm{C} / \mathrm{ha}$ ) & 135 & 90 & 135 & 90 \\
\hline
\end{tabular}


Houghton \& Hackler 2001, CDIAC NDP-050/R1 (http://cdiac.esd.ornl.gov/ndps/ndp050.html)

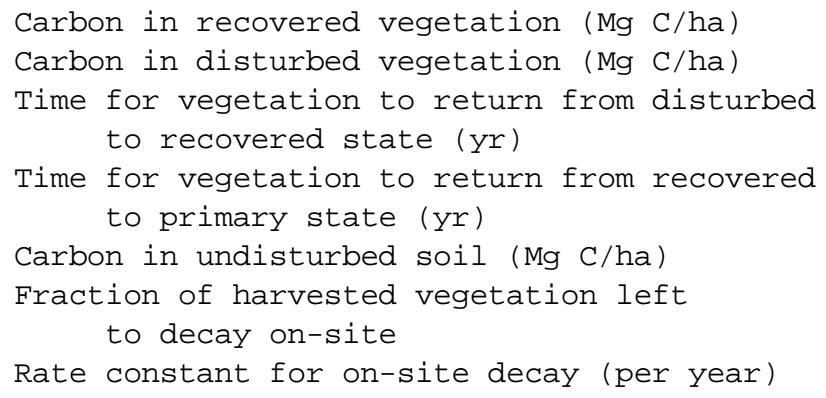

$\begin{array}{rlrc}108 & 72 & 108 & 72 \\ 97 & 46 & 0 & 0 \\ & & & \\ 14 & 29 & 40 & 80 \\ & & & \\ 20 & 24 & 20 & 24 \\ 134 & 206 & 134 & 206 \\ & & & \\ 0.20 & 0.21 & 0.37 & 0.30 \\ 0.04 & 0.05 & 0.04 & 0.05\end{array}$


Houghton \& Hackler 2001, CDIAC NDP-050/R1 (http://cdiac.esd.ornl.gov/ndps/ndp050.html)

Former Soviet Union - Partitioning of logging products into decay pools by harvest type, ecosystem type, and year:

\section{Fuelwood harvest}

\section{Temperate deciduous forest}

Decay pool

$1-\mathrm{yr}$

$10-\mathrm{yr}$

$100-y r$

$1000-y r$

\begin{tabular}{lrrrr}
\multicolumn{5}{c}{ Years } \\
\hline $1700-1944$ & $1945-1959$ & $1960-1974$ & $1975-1979$ & $1980-1990$ \\
0.71132 & 0.73342 & 0.59211 & 0.65211 & 0.64737 \\
0.00000 & 0.00000 & 0.00000 & 0.00000 & 0.00000 \\
0.07816 & 0.05605 & 0.19737 & 0.13737 & 0.14211 \\
0.00000 & 0.00000 & 0.00000 & 0.00000 & 0.00000
\end{tabular}

Years

\begin{tabular}{rrrrr}
\hline $1700-1944$ & $1945-1959$ & $1960-1974$ & $1975-1979$ & $1980-1990$ \\
0.71670 & 0.73898 & 0.59659 & 0.65705 & 0.65227 \\
0.00000 & 0.00000 & 0.00000 & 0.00000 & 0.00000 \\
0.07875 & 0.05648 & 0.19886 & 0.13841 & 0.14318 \\
0.00000 & 0.00000 & 0.00000 & 0.00000 & 0.00000
\end{tabular}

\section{Timber harvest}

Temperate deciduous forest

Decay pool
$1-y r$
$10-y r$
$100-y r$
$1000-y r$

Boreal forest

Decay pool
$1-y r$
$10-y r$
$100-y r$
$1000-y r$

\begin{tabular}{crccc}
\multicolumn{5}{c}{ Year } \\
\hline 1700 & 1945 & 1960 & 1975 & 1990 \\
0.21407 & 0.40296 & 0.18259 & 0.11333 & 0.09444 \\
0.00000 & 0.00000 & 0.00000 & 0.00000 & 0.00000 \\
0.41556 & 0.22667 & 0.44704 & 0.51630 & 0.53519 \\
0.00000 & 0.00000 & 0.00000 & 0.00000 & 0.00000
\end{tabular}

\begin{tabular}{crccc}
\multicolumn{5}{c}{ Year } \\
\hline 1700 & 1945 & 1960 & 1975 & 1990 \\
0.23611 & 0.44444 & 0.20139 & 0.12500 & 0.10417 \\
0.00000 & 0.00000 & 0.00000 & 0.00000 & 0.00000 \\
0.45833 & 0.25000 & 0.49306 & 0.56944 & 0.59028 \\
0.00000 & 0.00000 & 0.00000 & 0.00000 & 0.00000
\end{tabular}




\section{South and Central America (the following values replace the data in files scam_re.* in Houghton and Hackler 1995)}

\section{South and Central America — Clearing Response Curve:}

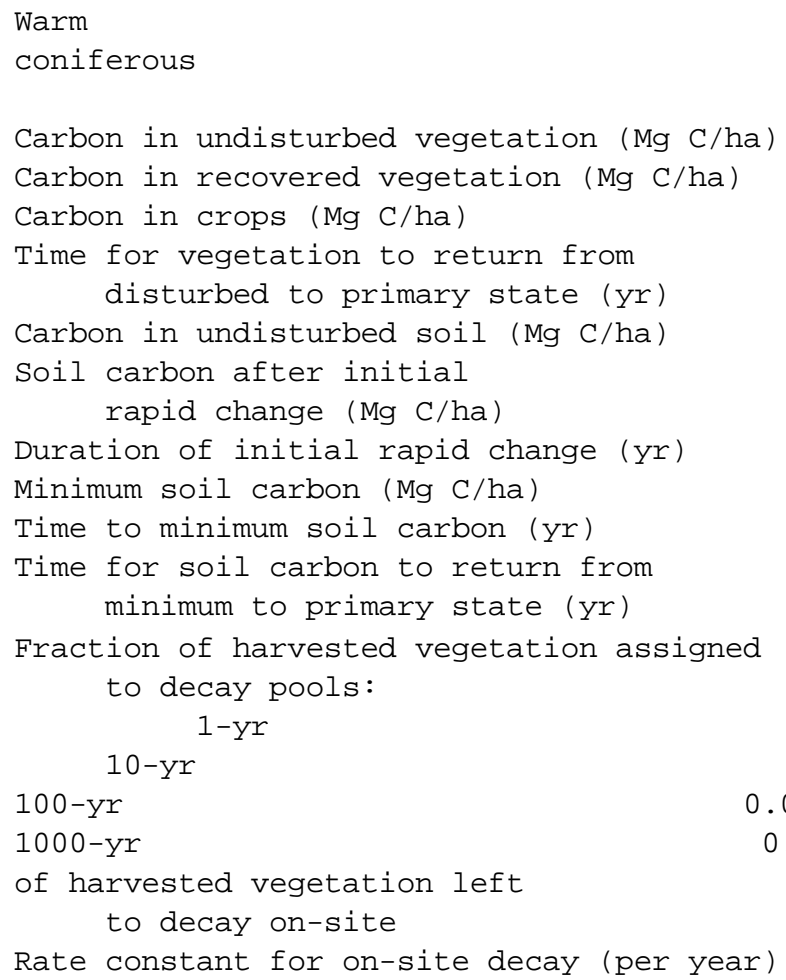

\begin{tabular}{rrrr}
\multicolumn{5}{c}{ Tropical } & Tropical \\
equatorial & seasonal & Tropical \\
forest & forest & woodland & forest \\
200 & 140 & 55 & 168 \\
200 & 140 & 55 & 168 \\
5 & 5 & 5 & 5 \\
40 & 35 & 18 & 42 \\
98 & 98 & 69 & 134 \\
78 & 78 & 55 & 107 \\
5 & 5 & 5 & 5 \\
74 & 74 & 52 & 100 \\
20 & 20 & 20 & 20 \\
40 & 35 & 18 & 42
\end{tabular}

$\begin{array}{lllll}0.35^{0.30} & 0.35^{0.30} & 0.30^{0.30} & 0.35^{0.30}\end{array}$

0.00

0.02

0.00

0.00

0.02

0.00

0.02

\begin{tabular}{cccc}
0.33 & 0.33 & 0.38 & 0.33 \\
0.50 & 0.40 & 0.30 & 0.30 \\
Temperate & & \\
broadleaf & Desert & \\
\multicolumn{2}{r}{ forest } & Grassland & scrub
\end{tabular}

100

100

5

25

134

107

5

100

20

40 
Houghton \& Hackler 2001, CDIAC NDP-050/R1 (http://cdiac.esd.ornl.gov/ndps/ndp050.html)

$\begin{array}{cccc}1-\mathrm{yr} & 0.30 & 0.48 & 0.48 \\ 10-\mathrm{yr} & 0.35 & 0.00 & 0.00 \\ 100-\mathrm{yr} & 0.00 & 0.00 & 0.00 \\ 1000-\mathrm{yr} & 0.02 & 0.02 & 0.02 \\ \text { Fraction of harvested vegetation left to decay on-site } & 0.33 & 0.50 & 0.50 \\ \text { Rate constant for on-site decay (per year) } & 0.50 & 0.30 & 0.20\end{array}$

South and Central America — Pasture Response Curve:

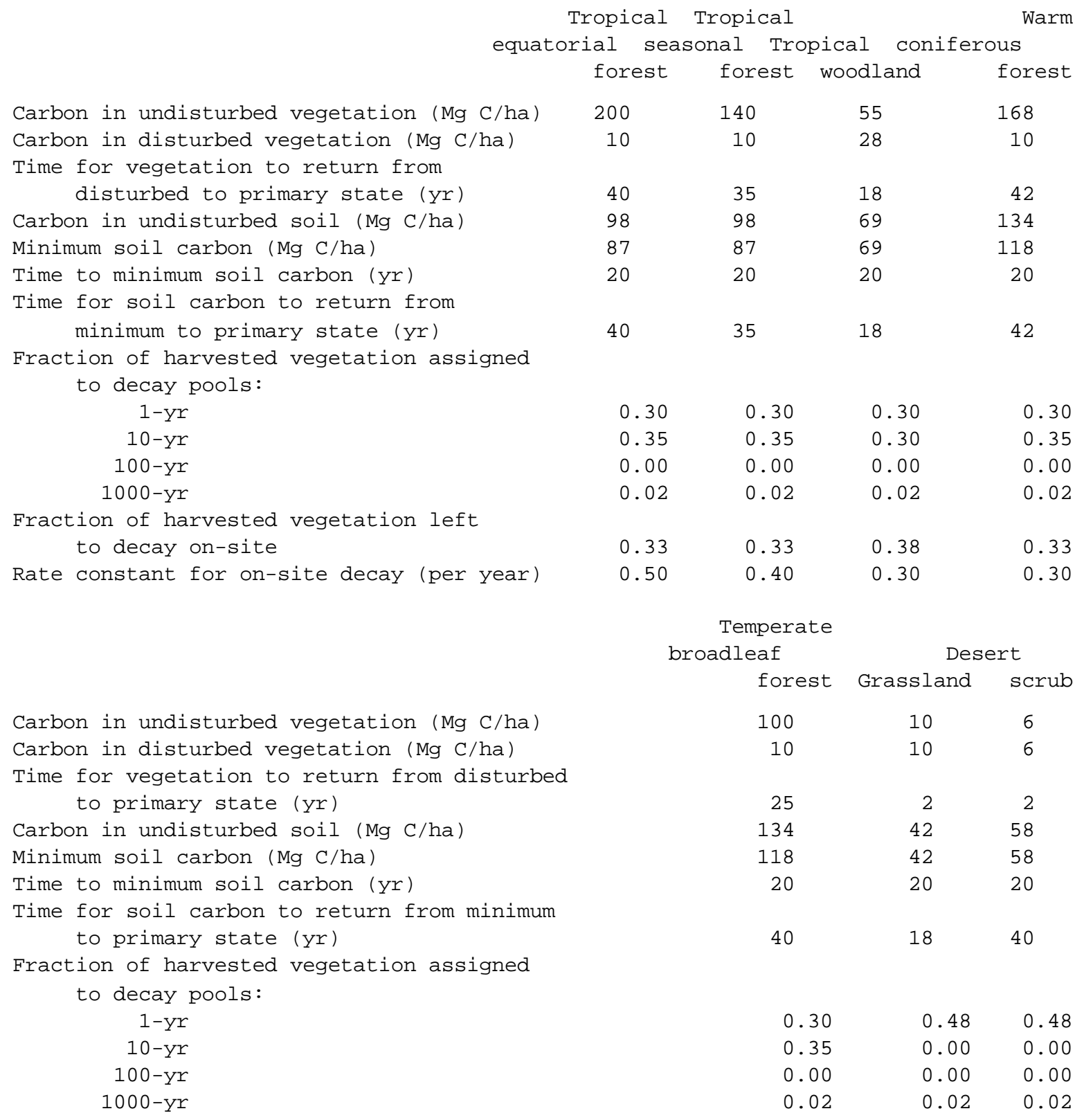


Houghton \& Hackler 2001, CDIAC NDP-050/R1 (http://cdiac.esd.ornl.gov/ndps/ndp050.html)

Fraction of harvested vegetation left to decay on-site

Rate constant for on-site decay (per year)

$\begin{array}{lll}0.33 & 0.50 & 0.50 \\ 0.50 & 0.40 & 0.30\end{array}$

C-11 


\section{South and Central America — Shifting Cultivation Response Curve:}

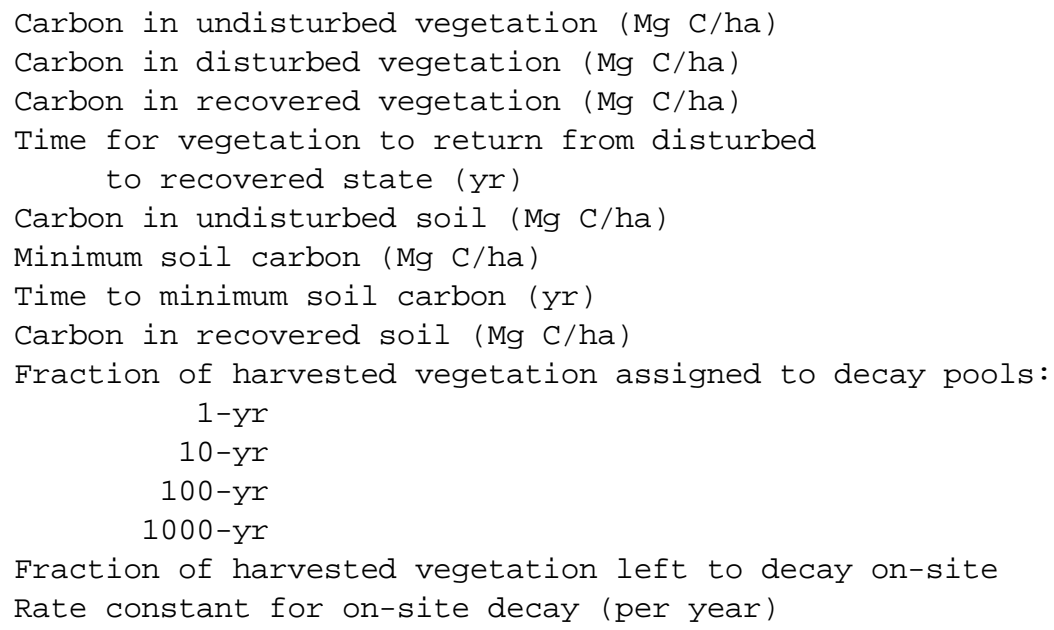

South and Central America — Logging Response Curve:

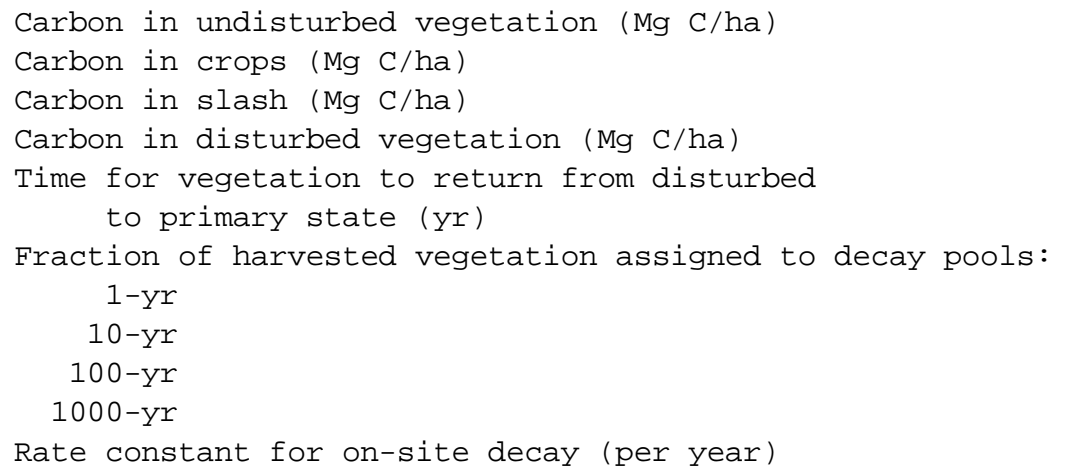

$\begin{array}{rr}\begin{array}{r}\text { Tropical } \\ \text { seasonal } \\ \text { forest }\end{array} & \begin{array}{r}\text { Coniferous } \\ \text { forest }\end{array} \\ 140 & 168 \\ 3 & 15 \\ 15 & 25 \\ 122 & 128 \\ & \\ 6 & 13 \\ 0.0 & 0.0 \\ 0.0 & 0.1 \\ 1.0 & 0.9 \\ 0.0 & 0.0 \\ 0.4 & 0.4\end{array}$




\section{South and Central America - Forest Plantation Response Curve:}

$\begin{array}{lr} & \begin{array}{r}\text { Tropical } \\ \text { seasonal } \\ \text { forest }\end{array} \\ \text { Carbon in undisturbed vegetation (Mg C/ha) } & 140 \\ \text { Carbon in disturbed vegetation (Mg C/ha) } & 10 \\ \text { Carbon in undisturbed soil (Mg C/ha) } & 98 \\ \text { Minimum soil carbon (Mg C/ha) } & 87 \\ \text { Time to minimum soil carbon (yr) } & 2 \\ \text { Fraction of harvested vegetation assigned to decay pools: } & 0 \\ \text { 1-yr } & 0 \\ \text { 10-yr } & 0 \\ \text { 100-yr } & 0 \\ \text { Fraction of harvested vegetation left to decay on-site } & 1 \\ \text { Rate constant for on-site decay (per year) } & 0.4\end{array}$

Tropical Africa (the following values replace the data in files taf_re.* in Houghton and Hackler 1995)

Tropical Africa - Clearing response curve:

\begin{tabular}{|c|c|c|}
\hline & $\begin{array}{l}\text { Closed } \\
\text { Forest }\end{array}$ & $\begin{array}{r}\text { Open } \\
\text { forest }\end{array}$ \\
\hline Carbon in undisturbed vegetation ( $\mathrm{Mg} \mathrm{C} / \mathrm{ha}$ ) & 136 & 30 \\
\hline Carbon in recovered vegetation ( $\mathrm{Mg} \mathrm{C} / \mathrm{ha}$ ) & 136 & 30 \\
\hline Carbon in crops (Mg C/ha) & 15 & 15 \\
\hline Time for vegetation to return from disturbed to recovered state (yr) & 30 & 30 \\
\hline Carbon in undisturbed soil (Mg C/ha) & 100 & 50 \\
\hline Carbon in recovered soil (Mg $\mathrm{C} / \mathrm{ha}$ ) & 100 & 50 \\
\hline Soil carbon after initial rapid change ( $\mathrm{Mg} \mathrm{C} / \mathrm{ha}$ ) & 80 & 40 \\
\hline Minimum soil carbon (Mg C/ha) & 75 & 37 \\
\hline Duration of initial rapid change (yr) & 1 & 1 \\
\hline Time to minimum soil carbon (yr) & 5 & 5 \\
\hline $\begin{array}{l}\text { Time for soil carbon to return from minimum to recovered state (yr) } \\
\text { Fraction of harvested vegetation assigned to decay pools: }\end{array}$ & 30 & 30 \\
\hline $1-\mathrm{yr}$ & 0.40 & 0.40 \\
\hline $10-\mathrm{yr}$ & 0.27 & 0.27 \\
\hline $100-y r$ & 0.00 & 0.00 \\
\hline $1000-y r$ & 0.00 & 0.00 \\
\hline Fraction of harvested vegetation left to decay on-site & 0.33 & 0.33 \\
\hline Rate constant for on-site decay (pe & 0.50 & 0.30 \\
\hline
\end{tabular}


Houghton \& Hackler 2001, CDIAC NDP-050/R1 (http://cdiac.esd.ornl.gov/ndps/ndp050.html)

North America, China, Europe, Pacific Developed Region, and North Africa and the Middle East (the following values replace the data in files tem_re.* in Houghton and Hackler 1995)

North America, China, Europe, Pacific Developed Region, and North Africa and the Middle East Clearing Response Curve:

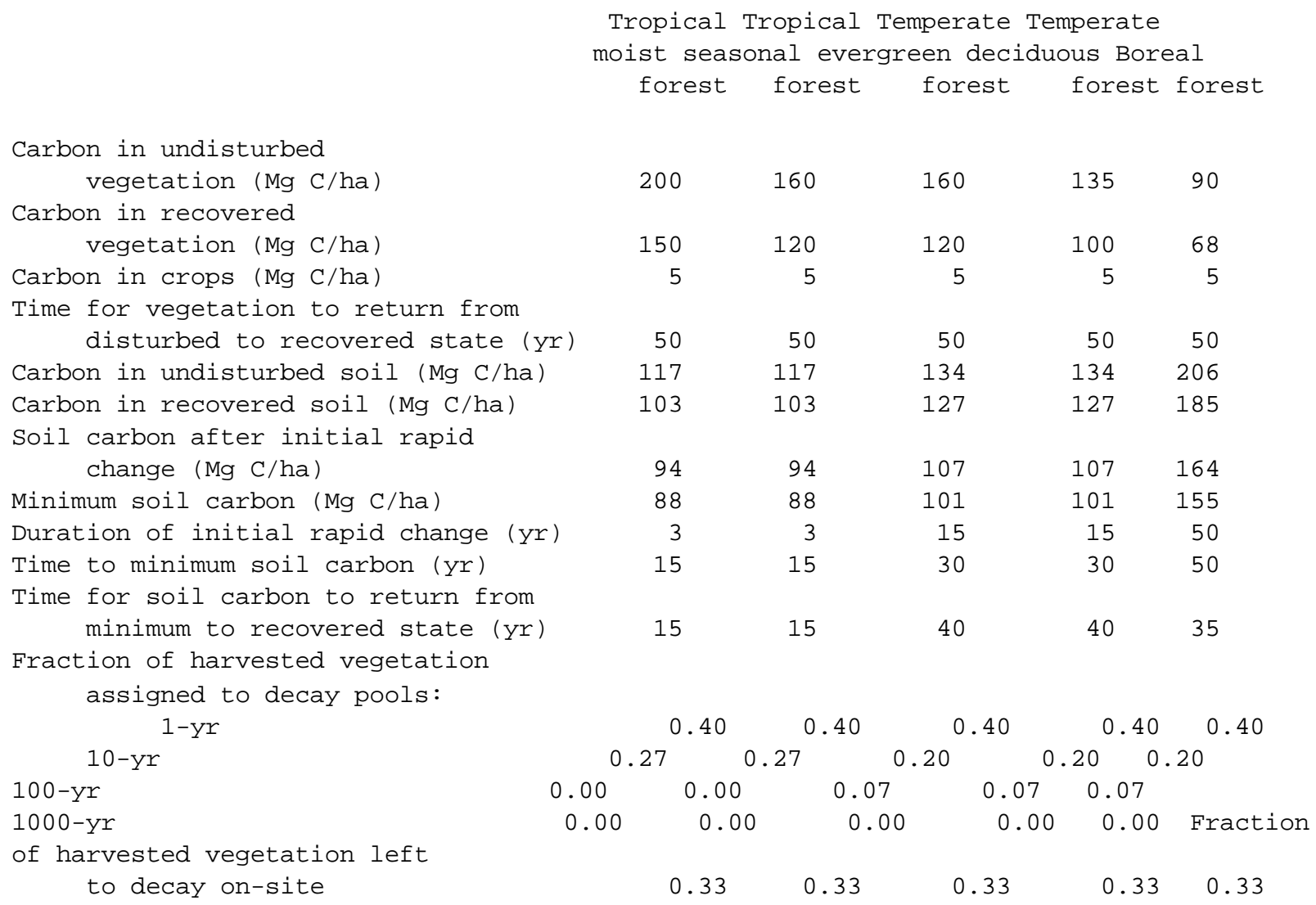


North America, China, Europe, Pacific Developed Region, and North Africa and the Middle East Clearing Response Curve (continued)

\begin{tabular}{|c|c|c|c|c|c|}
\hline & $\begin{array}{l}\text { Tropical } \\
\text { woodland/ } \\
\text { shrubland }\end{array}$ & $\begin{array}{l}\text { Temperate } \\
\text { woodland/ } \\
\text { shrubland }\end{array}$ & $\begin{array}{l}\text { Tropical } \\
\text { grassland }\end{array}$ & $\begin{array}{l}\text { Temperate } \\
\text { grassland }\end{array}$ & $\begin{array}{r}\text { Desert } \\
\text { scrub }\end{array}$ \\
\hline \multicolumn{6}{|l|}{ Carbon in undisturbed } \\
\hline $\begin{array}{l}\text { vegetation ( } \mathrm{Mg} \mathrm{C} / \mathrm{ha}) \\
\text { Carbon in recovered }\end{array}$ & 27 & 27 & 18 & 7 & 3 \\
\hline vegetation (Mg C/ha) & 27 & 27 & 18 & 7 & 3 \\
\hline Carbon in crops ( $\mathrm{Mg} \mathrm{C} / \mathrm{ha}$ ) & 5 & 5 & 5 & 3 & 1 \\
\hline \multicolumn{6}{|l|}{$\begin{array}{l}\text { Time for vegetation to return from } \\
\text { disturbed to recovered }\end{array}$} \\
\hline state (yr) & 25 & 50 & 5 & 10 & 10 \\
\hline Carbon in undisturbed soil ( $\mathrm{Mg} \mathrm{C} / \mathrm{ha}$ ) & 69 & 69 & 42 & 189 & 58 \\
\hline Carbon in recovered soil (Mg C/ha) & 69 & 69 & 42 & 189 & 58 \\
\hline \multicolumn{6}{|l|}{ Soil carbon after initial rapid } \\
\hline Minimum soil carbon (Mg $\mathrm{C} / \mathrm{ha}$ ) & 52 & 52 & 32 & 142 & 87 \\
\hline Duration of initial rapid change (yr) & 3 & 15 & 3 & 15 & 5 \\
\hline Time to minimum soil carbon (yr) & 15 & 30 & 15 & 30 & 10 \\
\hline $\begin{array}{l}\text { Time for soil carbon to return from } \\
\text { minimum to recovered state (yr) }\end{array}$ & 15 & 45 & 15 & 45 & 10 \\
\hline \multicolumn{6}{|l|}{$\begin{array}{c}\text { Fraction of harvested vegetation } \\
\text { assigned to decay pools: }\end{array}$} \\
\hline $1-\mathrm{yr}$ & 0.40 & 0.40 & 0.50 & 0.50 & 0.50 \\
\hline $10-y r$ & 0.10 & 0.10 & 0.00 & 0.00 & 0.00 \\
\hline $100-y r$ & 0.00 & 0.00 & 0.00 & 0.00 & 0.00 \\
\hline $1000-y r$ & 0.00 & 0.00 & 0.00 & 0.00 & 0.00 \\
\hline $\begin{array}{l}\text { Fraction of harvested vegetation left } \\
\text { to decay on-site }\end{array}$ & 0.50 & 0.50 & 0.50 & 0.50 & 0.50 \\
\hline
\end{tabular}

North America, China, Europe, Pacific Developed Region, and North Africa and the Middle East Logging Response Curve:

$\begin{array}{rrrr}\begin{array}{r}\text { Tropical } \\ \text { moist } \\ \text { forest }\end{array} & \begin{array}{r}\text { Temperate } \\ \text { evergreen } \\ \text { forest }\end{array} & \begin{array}{r}\text { Temperate } \\ \text { deciduous } \\ \text { forest }\end{array} & \begin{array}{r}\text { Boreal } \\ \text { forest }\end{array} \\ 200 & 160 & 135 & 90 \\ 150 & 120 & 100 & 68 \\ 0 & 0 & 0 & 0 \\ & & & \\ 50 & 50 & 50 & 50 \\ 117 & 134 & 134 & 206 \\ 103 & 127 & 127 & 185 \\ 76 & 108 & 108 & 165 \\ 73 & 42 & 51 & 17 \\ 127 & 118 & 84 & 73\end{array}$


Houghton \& Hackler 2001, CDIAC NDP-050/R1 (http://cdiac.esd.ornl.gov/ndps/ndp050.html)

Time to minimum soil carbon ( $\mathrm{y} r$ )

5

10

10

15

Time for soil carbon to return from minimum to recovered state (yr) 
Partitioning of logging products (from all ecosystem types) into decay pools by region and year:

\section{China}

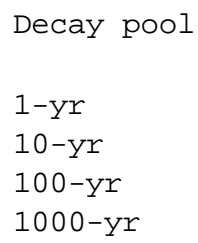

Europe

Decay pool

$1-\mathrm{yr}$

$10-y \mathrm{r}$

100-yr

1000-yr

\section{North Africa and the Middle East}

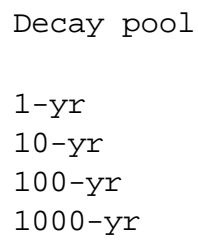

\section{North America}

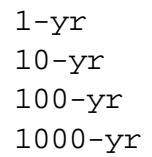

Decay pool

Pacific Developed Region

\begin{tabular}{llllll}
\hline 1700 & 1875 & 1900 & 1925 & 1950 & 1980 \\
& & & & & \\
0.90 & 0.90 & 0.90 & 0.86 & 0.80 & 0.75 \\
0.04 & 0.04 & 0.04 & 0.06 & 0.08 & 0.10 \\
0.06 & 0.06 & 0.06 & 0.08 & 0.12 & 0.15 \\
0.00 & 0.00 & 0.00 & 0.00 & 0.00 & 0.00
\end{tabular}

\begin{tabular}{llllll}
\hline \multicolumn{6}{c}{ Year } \\
\hline 1700 & 1875 & 1900 & 1925 & 1950 & 1980 \\
0.50 & 0.50 & 0.50 & 0.48 & 0.42 & 0.18 \\
0.20 & 0.20 & 0.20 & 0.21 & 0.23 & 0.33 \\
0.30 & 0.30 & 0.30 & 0.31 & 0.35 & 0.49 \\
0.00 & 0.00 & 0.00 & 0.00 & 0.00 & 0.00
\end{tabular}

\begin{tabular}{llllll}
\hline \multicolumn{6}{c}{ Year } \\
\hline 1700 & 1875 & 1900 & 1925 & 1950 & 1980 \\
0.90 & 0.90 & 0.90 & 0.87 & 0.75 & 0.63 \\
0.04 & 0.04 & 0.04 & 0.05 & 0.10 & 0.15 \\
0.06 & 0.06 & 0.06 & 0.08 & 0.15 & 0.22 \\
0.00 & 0.00 & 0.00 & 0.00 & 0.00 & 0.00
\end{tabular}

Year

\begin{tabular}{llllll}
\hline 1700 & 1875 & 1900 & 1925 & 1950 & 1980 \\
0.50 & 0.44 & 0.40 & 0.30 & 0.20 & 0.04 \\
0.20 & 0.22 & 0.24 & 0.28 & 0.32 & 0.38 \\
0.30 & 0.34 & 0.36 & 0.42 & 0.48 & 0.58 \\
0.00 & 0.00 & 0.00 & 0.00 & 0.00 & 0.00
\end{tabular}




\begin{tabular}{llllll}
\hline \multicolumn{6}{c}{ Year } \\
\hline 1700 & 1875 & 1900 & 1925 & 1950 & 1980 \\
0.81 & 0.81 & 0.81 & 0.81 & 0.42 & 0.27 \\
0.08 & 0.08 & 0.08 & 0.08 & 0.23 & 0.29 \\
0.11 & 0.11 & 0.11 & 0.11 & 0.35 & 0.44 \\
0.00 & 0.00 & 0.00 & 0.00 & 0.00 & 0.00
\end{tabular}

\section{APPENDIX D. FULL LISTING OF NDP050.DAT (FILE 2)}

The following is a full listing of ascii file ndp050.dat (File 2), which is also provided, in binary spreadsheet format, as file ndp050.wk1 (File 3). This file lists the estimated net flux of carbon, in units of $1000 \mathrm{Mg}$ of carbon ( 1 megagram $=10^{6} \mathrm{~g}$ ), to the atmosphere from land-use change, from 1850 through 1990, by year and by region, along with the global totals. The values in this listing replace the values in files netflux.* in Houghton and Hackler (1995), the previous version of this database.

\begin{tabular}{|c|c|c|c|c|c|c|c|c|c|c|}
\hline & & outh and & & North & & Former & & South and & Pacific & \\
\hline & North & Central & & Africa and & Tropical & Soviet & & Southeast & Developed & TOTAL \\
\hline Year & America & America & Europe & Middle East & Africa & Union & China & Asia & Region & FLUX \\
\hline 1850 & 87.28 & 42.48 & 55.04 & 3.98 & 5.61 & 58.56 & 56.52 & 85.63 & 2.05 & 397.145 \\
\hline 1851 & 87.22 & 42.18 & 55.02 & 3.98 & 6.47 & 58.55 & 56.50 & 85.20 & 2.04 & 397.164 \\
\hline 1852 & 90.37 & 41.90 & 54.99 & 3.98 & 6.60 & 58.88 & 56.48 & 85.25 & 2.04 & 400.492 \\
\hline 1853 & 93.38 & 41.66 & 54.96 & 3.98 & 6.69 & 59.22 & 56.47 & 85.33 & 2.03 & 403.713 \\
\hline 1854 & 96.28 & 41.44 & 54.93 & 3.98 & 6.77 & 59.58 & 56.45 & 85.42 & 2.03 & 406.877 \\
\hline 1855 & 99.12 & 41.24 & 54.90 & 3.98 & 6.82 & 59.96 & 56.43 & 85.53 & 2.03 & 410.017 \\
\hline 1856 & 101.93 & 41.06 & 54.87 & 3.98 & 6.86 & 60.34 & 56.42 & 85.65 & 2.02 & 413.137 \\
\hline 1857 & 104.72 & 40.91 & 54.84 & 3.98 & 6.88 & 60.72 & 56.40 & 85.77 & 2.02 & 416.250 \\
\hline 1858 & 107.50 & 40.77 & 54.81 & 3.98 & 6.90 & 61.10 & 56.39 & 85.90 & 2.01 & 419.363 \\
\hline 1859 & 110.27 & 40.64 & 54.78 & 3.98 & 6.92 & 61.48 & 56.37 & 86.02 & 2.01 & 422.481 \\
\hline 1860 & 113.06 & 38.09 & 54.75 & 3.98 & 6.93 & 61.85 & 56.36 & 86.14 & 2.01 & 423.171 \\
\hline 1861 & 116.34 & 32.46 & 54.79 & 9.01 & 8.02 & 62.22 & 56.25 & 86.26 & 2.08 & 427.422 \\
\hline 1862 & 119.70 & 29.66 & 54.86 & 9.96 & 8.18 & 53.69 & 56.13 & 86.38 & 2.18 & 420.740 \\
\hline 1863 & 123.16 & 26.89 & 54.96 & 10.82 & 8.31 & 53.75 & 55.99 & 86.49 & 2.30 & 422.672 \\
\hline 1864 & 126.70 & 24.20 & 55.06 & 11.25 & 8.41 & 53.80 & 55.85 & 86.61 & 2.43 & 424.303 \\
\hline 1865 & 130.31 & 21.57 & 55.18 & 11.64 & 8.49 & 53.86 & 55.71 & 86.71 & 2.56 & 426.033 \\
\hline 1866 & 131.46 & 20.62 & 55.31 & 11.99 & 8.53 & 53.74 & 55.55 & 86.82 & 2.70 & 426.713 \\
\hline 1867 & 132.67 & 19.74 & 55.46 & 12.31 & 8.57 & 53.62 & 55.39 & 86.91 & 2.83 & 427.495 \\
\hline 1868 & 133.95 & 18.90 & 55.61 & 12.61 & 8.59 & 53.51 & 55.23 & 87.01 & 2.96 & 428.377 \\
\hline 1869 & 135.30 & 18.11 & 55.78 & 12.89 & 8.62 & 53.41 & 55.06 & 87.10 & 3.09 & 429.350 \\
\hline 1870 & 136.72 & 19.79 & 55.95 & 13.15 & 8.64 & 53.32 & 54.88 & 87.18 & 3.21 & 432.843 \\
\hline 1871 & 138.16 & 23.97 & 50.48 & 13.40 & 8.66 & 53.23 & 54.70 & 108.79 & 9.87 & 461.264 \\
\hline 1872 & 139.64 & 25.66 & 49.60 & 13.64 & 8.67 & 53.49 & 54.53 & 113.96 & 11.93 & 471.118 \\
\hline 1873 & 141.14 & 27.32 & 48.75 & 13.87 & 8.69 & 53.75 & 54.35 & 117.71 & 13.66 & 479.236 \\
\hline 1874 & 142.67 & 28.92 & 47.95 & 14.09 & 8.70 & 54.01 & 51.69 & 120.51 & 14.33 & 482.862 \\
\hline 1875 & 144.23 & 30.47 & 47.17 & 14.30 & 8.71 & 54.27 & 51.15 & 122.69 & 14.87 & 487.850 \\
\hline 1876 & 144.64 & 30.66 & 46.53 & 14.47 & 9.79 & 54.94 & 50.74 & 124.08 & 15.35 & 491.204 \\
\hline 1877 & 144.91 & 31.36 & 45.94 & 14.63 & 9.96 & 55.04 & 50.41 & 125.23 & 15.76 & 493.231 \\
\hline 1878 & 145.05 & 32.02 & 45.39 & 14.79 & 10.08 & 55.13 & 50.11 & 126.23 & 16.12 & 494.901 \\
\hline 1879 & 145.06 & 32.63 & 44.87 & 14.93 & 10.18 & 55.21 & 49.84 & 127.11 & 16.44 & 496.273 \\
\hline 1880 & 144.94 & 33.22 & 44.39 & 15.07 & 10.26 & 55.30 & 49.59 & 127.91 & 16.75 & 497.425 \\
\hline 1881 & 144.70 & 70.55 & 43.94 & 15.21 & 10.30 & 55.40 & 49.35 & 128.27 & 17.05 & 534.771 \\
\hline 1882 & 144.34 & 82.30 & 43.52 & 15.35 & 10.33 & 55.51 & 49.14 & 128.59 & 17.34 & 546.421 \\
\hline 1883 & 143.86 & 92.24 & 43.12 & 15.49 & 10.36 & 55.63 & 48.94 & 128.87 & 17.64 & 556.158 \\
\hline 1884 & 143.27 & 100.88 & 42.74 & 15.63 & 10.39 & 55.76 & 48.75 & 129.13 & 17.94 & 564.499 \\
\hline 1885 & 142.58 & 108.58 & 42.39 & 15.77 & 10.41 & 55.90 & 48.58 & 129.37 & 18.24 & 571.807 \\
\hline 1886 & 141.82 & 112.04 & 42.56 & 15.90 & 11.71 & 56.03 & 48.42 & 129.59 & 18.54 & 576.593 \\
\hline 1887 & 141.00 & 114.43 & 42.73 & 16.03 & 11.91 & 56.17 & 48.26 & 129.79 & 18.83 & 579.162 \\
\hline 1888 & 140.14 & 116.44 & 42.92 & 16.16 & 12.06 & 56.30 & 48.11 & 129.98 & 19.12 & 581.235 \\
\hline
\end{tabular}


Houghton \& Hackler 2001, CDIAC NDP-050/R1 (http://cdiac.esd.ornl.gov/ndps/ndp050.html)

\begin{tabular}{|c|c|c|c|c|c|c|c|c|c|c|}
\hline 1889 & 139.21 & 118.15 & 43.11 & 16.29 & 12.18 & 56.44 & 48.04 & 130.16 & 19.36 & 582.940 \\
\hline 1890 & 138.09 & 119.63 & 43.31 & 16.42 & 12.28 & 56.57 & 47.97 & 130.32 & 19.60 & 584.180 \\
\hline 1891 & 148.45 & 92.35 & 43.51 & 16.54 & 12.34 & 56.70 & 47.90 & 146.76 & 19.83 & 584.388 \\
\hline 1892 & 150.16 & 84.19 & 43.72 & 16.66 & 12.38 & 56.83 & 47.84 & 150.78 & 20.06 & 582.620 \\
\hline 1893 & 151.73 & 77.09 & 43.91 & 16.78 & 12.42 & 56.96 & 47.79 & 153.67 & 20.28 & 580.630 \\
\hline 1894 & 153.18 & 70.78 & 44.11 & 16.90 & 12.45 & 57.08 & 47.75 & 155.83 & 20.50 & 578.581 \\
\hline 1895 & 154.55 & 65.05 & 44.31 & 17.01 & 12.47 & 57.21 & 47.70 & 157.53 & 20.72 & 576.549 \\
\hline 1896 & 155.52 & 63.60 & 44.50 & 17.12 & 12.50 & 57.33 & 47.66 & 158.61 & 20.93 & 577.764 \\
\hline 1897 & 156.43 & 62.47 & 44.68 & 17.23 & 12.52 & 57.45 & 47.63 & 159.49 & 21.14 & 579.038 \\
\hline 1898 & 157.30 & 61.59 & 44.86 & 17.34 & 12.53 & 57.57 & 47.60 & 160.22 & 21.35 & 580.364 \\
\hline 1899 & 158.14 & 60.90 & 45.03 & 17.45 & 12.55 & 57.68 & 47.57 & 160.87 & 21.55 & 581.740 \\
\hline 1900 & 158.92 & 60.36 & 45.19 & 17.55 & 12.56 & 57.79 & 47.54 & 161.44 & 21.75 & 583.112 \\
\hline 1901 & 159.83 & 124.92 & 45.44 & 20.07 & 15.57 & 57.90 & 47.62 & 161.69 & 22.03 & 655.075 \\
\hline 1902 & 160.75 & 144.42 & 45.70 & 20.58 & 16.02 & 58.05 & 47.72 & 161.98 & 22.34 & 677.559 \\
\hline 1903 & 161.70 & 160.67 & 45.98 & 21.04 & 16.35 & 58.19 & 47.84 & 162.24 & 22.65 & 696.656 \\
\hline 1904 & 162.67 & 174.59 & 46.28 & 21.29 & 16.61 & 58.33 & 47.96 & 162.47 & 22.97 & 713.179 \\
\hline 1905 & 163.67 & 186.84 & 46.60 & 21.52 & 16.82 & 58.47 & 48.11 & 162.67 & 23.30 & 727.993 \\
\hline 1906 & 163.10 & 191.39 & 46.93 & 21.72 & 16.93 & 58.59 & 48.25 & 170.77 & 23.62 & 741.299 \\
\hline & & outh and & & North & & Former & & South and & Pacific & \\
\hline & North & Central & & Africa and & Tropical & Soviet & & Southeast & Developed & TOTAL \\
\hline Year & America & America & Europe & Middle East & Africa & Union & China & Asia & Region & FLUX \\
\hline 1907 & 162.56 & 195.05 & 47.27 & 21.89 & 17.02 & 58.59 & 48.40 & 172.83 & 23.94 & 747.540 \\
\hline 1908 & 162.04 & 198.06 & 47.62 & 22.05 & 17.10 & 58.58 & 48.55 & 174.30 & 24.25 & 752.540 \\
\hline 1909 & 161.56 & 200.56 & 47.98 & 22.20 & 17.16 & 58.56 & 48.70 & 175.40 & 24.56 & 756.671 \\
\hline 1910 & 161.10 & 202.27 & 48.35 & 22.33 & 17.21 & 58.54 & 48.85 & 176.27 & 24.87 & 759.791 \\
\hline 1911 & 160.68 & 140.60 & 48.72 & 22.48 & 21.53 & 58.53 & 49.01 & 176.84 & 25.15 & 703.522 \\
\hline 1912 & 160.30 & 122.37 & 49.10 & 22.59 & 22.20 & 58.51 & 49.16 & 177.30 & 25.41 & 686.940 \\
\hline 1913 & 159.97 & 107.21 & 49.47 & 22.69 & 22.69 & 63.68 & 49.30 & 156.93 & 25.67 & 657.631 \\
\hline 1914 & 159.68 & 94.19 & 49.85 & 22.79 & 23.08 & 65.23 & 55.07 & 152.36 & 25.91 & 648.173 \\
\hline 1915 & 159.44 & 82.67 & 50.23 & 22.88 & 23.40 & 66.59 & 56.06 & 149.05 & 26.16 & 636.474 \\
\hline 1916 & 159.22 & 78.80 & 50.66 & 22.93 & 23.57 & 67.89 & 56.94 & 153.45 & 26.39 & 639.850 \\
\hline 1917 & 159.03 & 75.71 & 51.09 & 22.97 & 23.71 & 69.21 & 57.66 & 153.09 & 26.62 & 639.076 \\
\hline 1918 & 158.88 & 73.19 & 51.51 & 23.01 & 23.82 & 70.49 & 58.32 & 152.93 & 26.84 & 638.978 \\
\hline 1919 & 158.76 & 71.09 & 51.92 & 23.03 & 23.92 & 71.76 & 58.95 & 152.69 & 27.05 & 639.181 \\
\hline 1920 & 158.68 & 66.46 & 52.32 & 23.05 & 24.00 & 73.06 & 59.55 & 152.43 & 27.26 & 636.822 \\
\hline 1921 & 158.64 & 104.94 & 52.77 & 23.06 & 28.35 & 74.38 & 60.11 & 152.08 & 27.57 & 681.924 \\
\hline 1922 & 158.64 & 114.93 & 53.20 & 23.07 & 29.04 & 75.75 & 60.66 & 151.74 & 27.79 & 694.822 \\
\hline 1923 & 158.67 & 122.76 & 53.62 & 23.08 & 29.56 & 77.06 & 61.17 & 151.80 & 28.00 & 705.725 \\
\hline 1924 & 158.75 & 129.12 & 54.03 & 23.09 & 29.97 & 78.40 & 61.61 & 151.87 & 28.18 & 715.011 \\
\hline 1925 & 158.85 & 134.47 & 54.42 & 23.09 & 30.30 & 79.78 & 62.10 & 151.96 & 28.35 & 723.319 \\
\hline 1926 & 158.29 & 135.88 & 54.08 & 31.70 & 30.49 & 81.20 & 62.42 & 147.46 & 28.57 & 730.099 \\
\hline 1927 & 157.65 & 136.87 & 53.62 & 33.63 & 30.64 & 82.53 & 62.72 & 146.83 & 28.81 & 733.300 \\
\hline 1928 & 157.22 & 137.47 & 53.05 & 35.36 & 30.77 & 83.10 & 62.98 & 146.53 & 29.05 & 735.537 \\
\hline 1929 & 131.20 & 137.78 & 52.36 & 35.79 & 30.87 & 83.70 & 63.09 & 146.44 & 29.30 & 710.527 \\
\hline 1930 & 124.19 & 141.11 & 51.56 & 36.12 & 30.97 & 84.30 & 63.17 & 146.47 & 29.54 & 707.444 \\
\hline 1931 & 117.44 & 157.80 & 50.66 & 36.74 & 35.33 & 84.91 & 63.25 & 146.66 & 29.79 & 722.578 \\
\hline 1932 & 110.89 & 163.27 & 49.66 & 37.31 & 36.02 & 85.52 & 63.31 & 146.89 & 30.04 & 722.897 \\
\hline 1933 & 104.46 & 167.92 & 48.56 & 37.82 & 36.55 & 86.15 & 63.36 & 147.15 & 30.28 & 722.256 \\
\hline 1934 & 98.14 & 171.97 & 47.36 & 38.30 & 36.96 & 84.55 & 67.10 & 147.42 & 30.53 & 722.339 \\
\hline 1935 & 91.89 & 175.55 & 46.08 & 38.75 & 37.30 & 82.80 & 67.92 & 147.70 & 30.78 & 718.768 \\
\hline 1936 & 85.51 & 177.38 & 44.76 & 39.18 & 37.49 & 80.90 & 68.63 & 165.10 & 31.02 & 729.964 \\
\hline 1937 & 79.19 & 179.58 & 43.40 & 39.59 & 37.65 & 78.84 & 69.19 & 169.48 & 31.26 & 728.172 \\
\hline 1938 & 72.92 & 181.57 & 42.01 & 39.98 & 37.78 & 76.69 & 69.70 & 172.75 & 31.50 & 724.900 \\
\hline 1939 & 66.67 & 183.40 & 40.59 & 40.35 & 37.89 & 74.51 & 70.17 & 175.29 & 31.73 & 720.611 \\
\hline 1940 & 60.51 & 185.09 & 39.15 & 40.71 & 37.99 & 71.15 & 70.61 & 177.32 & 31.96 & 714.481 \\
\hline 1941 & 54.31 & 194.01 & 37.69 & 41.03 & 53.04 & 54.42 & 71.04 & 182.27 & 32.19 & 719.998 \\
\hline 1942 & 48.15 & 198.34 & 36.23 & 41.35 & 55.30 & 47.51 & 71.44 & 187.87 & 32.41 & 718.624 \\
\hline 1943 & 42.05 & 202.54 & 34.77 & 41.65 & 56.99 & 40.84 & 71.84 & 193.64 & 32.63 & 716.943 \\
\hline 1944 & 39.63 & 206.70 & 33.31 & 41.89 & 58.30 & 34.35 & 72.21 & 199.44 & 32.84 & 718.664 \\
\hline 1945 & 37.25 & 210.70 & 31.86 & 42.13 & 59.36 & 28.04 & 72.58 & 205.19 & 33.04 & 720.135 \\
\hline 1946 & 34.91 & 214.35 & 30.40 & 42.35 & 59.93 & 24.03 & 72.93 & 281.30 & 33.24 & 793.441 \\
\hline 1947 & 32.61 & 217.62 & 28.96 & 42.56 & 60.38 & 20.43 & 73.28 & 302.11 & 33.44 & 811.378 \\
\hline
\end{tabular}


Houghton \& Hackler 2001, CDIAC NDP-050/R1 (http://cdiac.esd.ornl.gov/ndps/ndp050.html)

\begin{tabular}{|c|c|c|c|c|c|c|c|c|c|c|}
\hline 1948 & 30.35 & 221.01 & 27.52 & 42.77 & 60.75 & 17.18 & 73.62 & 318.01 & 33.63 & 824.833 \\
\hline 1949 & 28.13 & 224.51 & 26.08 & 42.97 & 61.06 & 14.31 & 73.67 & 329.78 & 33.82 & 834.320 \\
\hline 1950 & 25.97 & 229.84 & 24.66 & 43.16 & 61.33 & 13.08 & 73.72 & 340.01 & 34.00 & 845.773 \\
\hline 1951 & 18.23 & 286.38 & 23.53 & 34.40 & 82.95 & 126.91 & 74.65 & 349.78 & 67.51 & 1064.327 \\
\hline 1952 & 15.04 & 304.72 & 22.55 & 32.64 & 86.28 & 149.64 & 75.60 & 362.04 & 76.88 & 1125.376 \\
\hline 1953 & 12.12 & 319.58 & 21.71 & 30.83 & 88.76 & 171.48 & 76.56 & 322.62 & 84.52 & 1128.181 \\
\hline 1954 & 9.44 & 331.78 & 21.01 & 28.61 & 90.71 & 192.67 & 77.52 & 323.13 & 86.59 & 1161.448 \\
\hline 955 & 6.97 & 342.05 & 20.44 & 26.39 & 92.29 & 213.75 & 97.76 & 326.78 & 87.92 & 1214.345 \\
\hline 1956 & 4.71 & 348.10 & 20.00 & 25.71 & 93.16 & 236.15 & 102.65 & 326.49 & 88.76 & 1245.711 \\
\hline 1957 & 2.63 & 353.04 & 19.68 & 25.04 & 93.86 & 258.25 & 107.01 & 326.34 & 89.26 & 1275.109 \\
\hline 1958 & 0.72 & 357.15 & 19.48 & 24.41 & 94.43 & 279.95 & 110.54 & 320.70 & 89.52 & 1296.919 \\
\hline 1959 & -1.02 & 362.41 & 19.40 & 23.79 & 94.92 & 208.34 & 113.79 & 320.51 & 89.62 & 1231.770 \\
\hline 1960 & -2.61 & 366.65 & 19.44 & 23.21 & 95.34 & 210.89 & 116.84 & 321.34 & 89.59 & 1240.677 \\
\hline 1961 & -4.09 & 481.93 & 16.70 & 22.64 & 129.92 & 205.22 & 119.69 & 325.96 & 89.48 & 1387.432 \\
\hline 1962 & -5.48 & 517.55 & 16.32 & 22.08 & 135.24 & 201.87 & 122.35 & 332.01 & 89.30 & 1431.247 \\
\hline 1963 & -6.76 & 546.07 & 16.01 & 21.55 & 139.22 & 198.82 & 124.84 & 347.12 & 89.09 & 1475.961 \\
\hline 1964 & -7.96 & 572.04 & 15.78 & 21.03 & 142.32 & 196.18 & 127.34 & 356.56 & 88.83 & 1512.116 \\
\hline 1965 & -9.08 & 593.94 & 15.61 & 20.52 & 144.85 & 192.92 & 129.51 & 365.27 & 88.56 & 1542.088 \\
\hline 1966 & -9.07 & 603.87 & 15.48 & 21.63 & 146.24 & 181.17 & 129.61 & 374.12 & 88.25 & 1551.292 \\
\hline 1967 & -9.03 & 612.03 & 15.39 & 21.82 & 147.36 & 169.52 & 130.71 & 382.93 & 87.94 & 1558.677 \\
\hline 1968 & -8.97 & 619.05 & 15.35 & 21.96 & 148.28 & 157.92 & 131.78 & 397.51 & 87.61 & 1570.492 \\
\hline 1969 & -8.87 & 629.61 & 15.33 & 21.73 & 149.06 & 147.84 & 132.89 & 407.46 & 87.04 & 1582.089 \\
\hline 1970 & -9.26 & 638.01 & 13.57 & 21.48 & 149.73 & 114.46 & 132.78 & 416.75 & 86.46 & 1563.981 \\
\hline 1971 & -9.53 & 567.12 & 11.18 & 21.52 & 184.52 & 100.10 & 104.98 & 407.73 & 50.23 & 1437.845 \\
\hline 1972 & -9.76 & 547.71 & 8.64 & 21.57 & 190.04 & 85.37 & 101.07 & 410.06 & 39.11 & 1393.820 \\
\hline 1973 & -9.93 & 531.05 & 5.96 & 21.62 & 194.18 & 71.25 & 97.25 & 474.68 & 29.74 & 1415.799 \\
\hline 1974 & -9.56 & 516.35 & 3.15 & 21.66 & 197.43 & 64.85 & 93.70 & 492.08 & 26.12 & 1405.787 \\
\hline 1975 & -9.15 & 503.09 & 0.21 & 21.71 & 200.09 & 58.78 & 90.12 & 505.73 & 23.24 & 1393.830 \\
\hline 1976 & -8.74 & 501.64 & -2.64 & 21.75 & 201.60 & 55.92 & 86.52 & 619.65 & 20.85 & 1496.554 \\
\hline
\end{tabular}

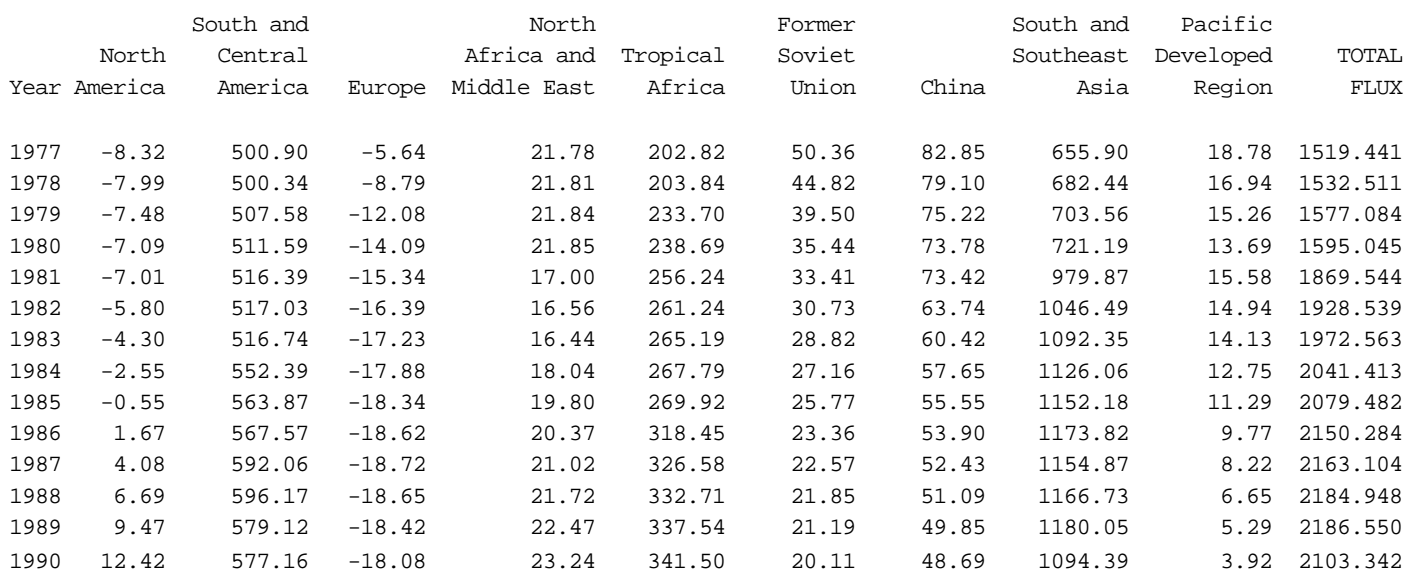




\section{APPENDIX E. FULL LISTING OF COMPARE.DAT (FILE 4)}

The following is a full listing of ascii file compare.dat (File 4), which is also provided, in binary spreadsheet format, as file compare.wk1 (File 5). This file compares the estimated global total net flux of carbon to the atmosphere from land-use change, from 1850 to 1990, by year, for this database (Houghton 1999) and three earlier publications (Houghton et al. 1983, Houghton and Skole 1990, and Houghton and Hackler 1995). Note that the data for the period 1850 through 1859 attributed below to Houghton et al. (1983) were not actually presented in that publication but are present in the data used in that publication.

Units $=$ Pg of carbon $\left(1\right.$ petagram $=10^{15}$ grams $) ;$ ! 9.999 denotes missing value

\begin{tabular}{|c|c|c|c|c|c|c|c|}
\hline Year & & Houghton & & Houghton & & Houghton & Houghton \\
\hline & et & al. 1983 & $\varepsilon$ & Skole 1990 & $\&$ & Hackler 1995 & 1999 \\
\hline 1850 & & 0.458 & & 0.278 & & 0.352 & 0.397 \\
\hline 1851 & & 0.464 & & 0.319 & & 0.383 & 0.397 \\
\hline 1852 & & 0.469 & & 0.353 & & 0.397 & 0.400 \\
\hline 1853 & & 0.475 & & 0.379 & & 0.408 & 0.404 \\
\hline 1854 & & 0.480 & & 0.393 & & 0.417 & 0.407 \\
\hline 1855 & & 0.486 & & 0.424 & & 0.424 & 0.410 \\
\hline 1856 & & 0.492 & & 0.439 & & 0.430 & 0.413 \\
\hline 1857 & & 0.497 & & 0.452 & & 0.435 & 0.416 \\
\hline 1858 & & 0.503 & & 0.464 & & 0.439 & 0.419 \\
\hline 1859 & & 0.509 & & 0.475 & & 0.443 & 0.422 \\
\hline 1860 & & 0.539 & & 0.492 & & 0.446 & 0.423 \\
\hline 1861 & & 0.563 & & 0.485 & & 0.457 & 0.427 \\
\hline 1862 & & 0.587 & & 0.492 & & 0.463 & 0.421 \\
\hline 1863 & & 0.612 & & 0.500 & & 0.468 & 0.423 \\
\hline 1864 & & 0.623 & & 0.508 & & 0.474 & 0.424 \\
\hline 1865 & & 0.634 & & 0.516 & & 0.479 & 0.426 \\
\hline 1866 & & 0.641 & & 0.522 & & 0.481 & 0.427 \\
\hline 1867 & & 0.649 & & 0.528 & & 0.484 & 0.427 \\
\hline 1868 & & 0.656 & & 0.534 & & 0.486 & 0.428 \\
\hline 1869 & & 0.665 & & 0.541 & & 0.489 & 0.429 \\
\hline 1870 & & 0.684 & & 0.544 & & 0.492 & 0.433 \\
\hline 1871 & & 0.701 & & 0.553 & & 0.501 & 0.461 \\
\hline 1872 & & 0.717 & & 0.562 & & 0.507 & 0.471 \\
\hline 1873 & & 0.731 & & 0.566 & & 0.512 & 0.479 \\
\hline 1874 & & 0.744 & & 0.572 & & 0.513 & 0.483 \\
\hline 1875 & & 0.771 & & 0.579 & & 0.517 & 0.488 \\
\hline 1876 & & 0.792 & & 0.585 & & 0.521 & 0.491 \\
\hline 1877 & & 0.813 & & 0.589 & & 0.524 & 0.493 \\
\hline 1878 & & 0.834 & & 0.592 & & 0.526 & 0.495 \\
\hline 1879 & & 0.846 & & 0.594 & & 0.528 & 0.496 \\
\hline 1880 & & 0.857 & & 0.621 & & 0.529 & 0.497 \\
\hline
\end{tabular}


Houghton \& Hackler 2001, CDIAC NDP-050/R1 (http://cdiac.esd.ornl.gov/ndps/ndp050.html)

\begin{tabular}{|c|c|c|c|c|c|c|c|}
\hline 1881 & & 0.868 & & 0.639 & & 0.560 & 0.535 \\
\hline 1882 & & 0.878 & & 0.658 & & 0.569 & 0.546 \\
\hline 1883 & & 0.888 & & 0.669 & & 0.576 & 0.556 \\
\hline 1884 & & 0.897 & & 0.679 & & 0.582 & 0.564 \\
\hline 1885 & & 0.905 & & 0.686 & & 0.586 & 0.572 \\
\hline \multirow[t]{2}{*}{ Year } & & Houghton & & Houghton & & Houghton & Houghton \\
\hline & et & al. 1983 & $\varepsilon$ & Skole 1990 & $\&$ & Hackler 1995 & 1999 \\
\hline 1886 & & 0.910 & & 0.692 & & 0.590 & 0.577 \\
\hline 1887 & & 0.915 & & 0.696 & & 0.592 & 0.579 \\
\hline 1888 & & 0.919 & & 0.700 & & 0.593 & 0.581 \\
\hline 1889 & & 0.923 & & 0.702 & & 0.594 & 0.583 \\
\hline 1890 & & 0.936 & & 0.701 & & 0.593 & 0.584 \\
\hline 1891 & & 0.944 & & 0.694 & & 0.597 & 0.584 \\
\hline 1892 & & 0.951 & & 0.688 & & 0.597 & 0.583 \\
\hline 1893 & & 0.959 & & 0.683 & & 0.597 & 0.581 \\
\hline 1894 & & 0.966 & & 0.679 & & 0.597 & 0.579 \\
\hline 1895 & & 0.973 & & 0.676 & & 0.597 & 0.577 \\
\hline 1896 & & 0.979 & & 0.673 & & 0.597 & 0.578 \\
\hline 1897 & & 0.985 & & 0.672 & & 0.596 & 0.579 \\
\hline 1898 & & 0.991 & & 0.674 & & 0.596 & 0.580 \\
\hline 1899 & & 0.997 & & 0.676 & & 0.595 & 0.582 \\
\hline 1900 & & 1.027 & & 0.732 & & 0.596 & 0.583 \\
\hline 1901 & & 1.048 & & 0.765 & & 0.660 & 0.655 \\
\hline 1902 & & 1.070 & & 0.791 & & 0.678 & 0.678 \\
\hline 1903 & & 1.092 & & 0.811 & & 0.693 & 0.697 \\
\hline 1904 & & 1.100 & & 0.828 & & 0.705 & 0.713 \\
\hline 1905 & & 1.108 & & 0.844 & & 0.716 & 0.728 \\
\hline 1906 & & 1.113 & & 0.851 & & 0.726 & 0.741 \\
\hline 1907 & & 1.117 & & 0.856 & & 0.731 & 0.748 \\
\hline 1908 & & 1.122 & & 0.860 & & 0.735 & 0.753 \\
\hline 1909 & & 1.126 & & 0.862 & & 0.738 & 0.757 \\
\hline 1910 & & 1.133 & & 0.815 & & 0.740 & 0.760 \\
\hline 1911 & & 1.138 & & 0.788 & & 0.686 & 0.704 \\
\hline 1912 & & 1.143 & & 0.762 & & 0.670 & 0.687 \\
\hline 1913 & & 1.153 & & 0.762 & & 0.646 & 0.658 \\
\hline 1914 & & 1.158 & & 0.758 & & 0.639 & 0.648 \\
\hline 1915 & & 1.164 & & 0.750 & & 0.630 & 0.636 \\
\hline 1916 & & 1.167 & & 0.750 & & 0.634 & 0.640 \\
\hline 1917 & & 1.170 & & 0.749 & & 0.635 & 0.639 \\
\hline 1918 & & 1.173 & & 0.750 & & 0.637 & 0.639 \\
\hline 1919 & & 1.175 & & 0.751 & & 0.639 & 0.639 \\
\hline 1920 & & 1.177 & & 0.789 & & 0.641 & 0.637 \\
\hline 1921 & & 1.179 & & 0.808 & & 0.691 & 0.682 \\
\hline 1922 & & 1.181 & & 0.823 & & 0.707 & 0.695 \\
\hline 1923 & & 1.182 & & 0.836 & & 0.722 & 0.706 \\
\hline 1924 & & 1.184 & & 0.842 & & 0.734 & 0.715 \\
\hline
\end{tabular}

E-2 


$\begin{array}{lllll}1925 & 1.281 & 0.866 & 0.745 & 0.723 \\ 1926 & 1.360 & 0.875 & 0.753 & 0.730 \\ 1927 & 1.441 & 0.883 & 0.755 & 0.733 \\ 1928 & 1.507 & 0.866 & 0.757 & 0.736 \\ 1929 & 1.527 & 0.844 & 0.736 & 0.711 \\ 1930 & 1.548 & 0.856 & 0.730 & 0.707 \\ 1931 & 1.569 & 0.861 & 0.740 & 0.723 \\ 1932 & 1.591 & 0.862 & 0.737 & 0.723 \\ 1933 & 1.614 & 0.860 & 0.732 & 0.722 \\ 1934 & 1.637 & 0.857 & 0.730 & 0.722\end{array}$

Year Houghton et al. 1983

1935

1936

1937

1938

1939

1940

1941

1942

1943

1944

1945

1946

1947

1948

1949

1950

1951

1952

1953

1954

1955

1956

1957

1958

1959

1960

1961

1962

1963

1964

1965

1966

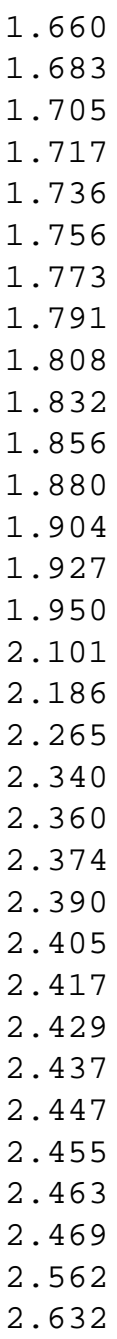

1. 660

1.705

1.717

1.736

1.756

1.773

1.808

1.832

1.856

1.880

1.904

1. 927

2. 101

2.265

2. 340

2. 360

2.374

2.390

2.405

2. 417

2.437

2.447

2.455

2.463

2. 469

2. 632
2.186
Houghton

\& Skole 1990

0.852

0.850

0.849

0.849

0.850

0.839

0.810

0.808

0.808

0.812

0.820

0.830

0.844

0.861

0.878

0.990

1. 150

1. 234

1.294

1. 418

1. 482

1. 528

1. 478

1. 483

1. 488

1.573

1.625

1.663

1.700

1.735

1.768

1. 747
Houghton

\& Hackler 1995

0.724

0.729

0.727

0.719

0.715

0.707

0.702

0.695

0.688

0.685

0.682

0.736

0.751

0.761

0.767

0.774

0.986

1.037

1.045

1.068

1.109

1.128

1.146

1.156

1.084

1.084

1. 217

1.255

1.293

1. 322

1. 348

1. 355
Houghton

1999

0.719

0.730

0.728

0.725

0.721

0.714

0.720

0.719

0.717

0.719

0.720

0.793

0.811

0.825

0.834

0.846

1.064

1.125

1.128

1. 161

1. 214

1. 246

1.275

1. 297

1. 232

1. 241

1. 387

1. 431

1.476

1. 512

1.542

1. 551 
Houghton \& Hackler 2001, CDIAC NDP-050/R1 (http://cdiac.esd.ornl.gov/ndps/ndp050.html)

$\begin{array}{lrrrr}1967 & 2.700 & 1.758 & 1.360 & 1.559 \\ 1968 & 2.768 & 1.775 & 1.368 & 1.570 \\ 1969 & 2.776 & 1.783 & 1.377 & 1.582 \\ 1970 & 2.721 & 1.684 & 1.357 & 1.564 \\ 1971 & 2.699 & 1.669 & 1.250 & 1.438 \\ 1972 & 2.677 & 1.632 & 1.215 & 1.394 \\ 1973 & 2.655 & 1.609 & 1.232 & 1.416 \\ 1974 & 2.653 & 1.567 & 1.233 & 1.406 \\ 1975 & 2.650 & 1.595 & 1.234 & 1.394 \\ 1976 & 2.646 & 1.611 & 1.324 & 1.497 \\ 1977 & 2.640 & 1.631 & 1.350 & 1.519 \\ 1978 & 2.633 & 1.616 & 1.367 & 1.533 \\ 1979 & 2.624 & 1.609 & 1.413 & 1.577 \\ 1980 & 2.613 & 1.608 & 1.423 & 1.595 \\ 1981 & -9.999 & 1.608 & 1.420 & 1.870 \\ 1982 & -9.999 & 1.608 & 1.450 & 1.929 \\ 1983 & -9.999 & 1.608 & 1.470 & 1.973\end{array}$

$\begin{array}{lrrrr}\text { Year } & \begin{array}{r}\text { Houghton } \\ \text { et } 1983\end{array} & \begin{array}{r}\text { Houghton } \\ \text { \& Skole } 1990\end{array} & \begin{array}{r}\text { Houghton } \\ \text { \& Hackler } 1995\end{array} & \text { Houghton } \\ 1999\end{array}$




\section{APPENDIX F: REPRINT OF PERTINENT LITERATURE}

Houghton, R. A. 1999. The annual net flux of carbon to the atmosphere from changes in land use 1850-1990. Tellus 51B:298-313.

Note: This electronic version of the report does not contain a copy of the reprint of the article. 